\title{
On the Index of Refraction of Air, the Absorption and Dispersion of Centimeter Waves by Gases
}

\author{
Georges Boudouris ${ }^{1}$
}

\author{
Contribution from A La Faculte Des Sciences De L'universite De Paris, translated from French \\ by G. Wm. Curtis
}

(Received May 31, 1963)

\begin{abstract}
The index of refraction, the absorption and dispersion are studied for several gases and vapors (pressure from 0 to $1 \mathrm{~atm}$, temperature from 0 to $50^{\circ} \mathrm{C}$, frequency from 7,000 to 12,000 $\mathrm{Mc} / \mathrm{s})$. The first part is devoted to a description of the microwave spectrometer used, while the second part is a survey of the several theories implied in the interpretation of the results.

In the third part, we present first the results concerning the index of refraction of atmospheric gases, of dry air, and of damp air. Then we study the absorption and dispersion by several vapors, and of ammonia and chloroform, principally. Finally, we indicate a new method making use of gaseous mixtures.

Our original results are discussed and presented within the frame of reference of works of other authors by means of comparison and intensive bibliographies.
\end{abstract}

\section{Experimental Setup}

\subsection{Introduction}

The experimental setups which have been perfected during the last $10 \mathrm{yr}$, in the field of hertzian spectroscopy, can be classified in three types of apparatus: waveguide spectrometers, resonating cavity spectrometers, nonresonating cavity spectrometers [Townes and Schalow, 1955 (chap. 15); Gordy et al., 1953 (chap. 1). If one desires the maximum of sensitivity and precision, one must, as we did, use a cavity spectrometer, and in particular, one equipped with two cavities, one cavity being used as a standard for frequencies, the other used as a container for the gas under study. The two cavities are used as transmission mediums; they are swept by the high-frequency energy of a klystron which is frequency modulated. They produce, after detection by a crystal, a resonance curve for each channel. The introduction of a gas in the cavity used for measurement displaces its response signal with respect to the response curve of the reference cavity (effect of the index of refraction $n$ of the gas); also this signal appears more or less flattened and weakened (effect of the absorption $\alpha$ of the gas on the quality factor of the cavity $Q$ ). A precise measurement of the deformation of the response curve will yield $n$ and $\alpha$. Such an interpretation of the response curve of the measurement cavity can be accomplished in many different manners. The most common one consists of flashing the response curves on the screen of an oscillograph where frequency standards appear at the same time. A microammeter has been equally used for absorption measurements.

An essential new feature of our instrument is the use of an impulse technique in the electronic circuitry

${ }_{1}$ Doctoral Thesis, present address: 1, Allee Yves du Manoir Chatenay-Malabry (Seine) France. which facilitates the interpretation of the response signals of the cavities. This technique, which will be discussed in greater detail later on, increases the sensitivity, the stability, and the precision of the instrument. This method has been proposed for the first time by A. Gozzini [1953] and his collaborators at the University of Pisa [Battaglia, Bruin, and Gozzini, 1958; Battaglia and Gozzini, 1955; Gozzini and Polacco, 1953a and 1953b]. This method has been developed and perfected later on; and a description of the present version of the instrument was presented in the communication which we gave at the last Congress on Hyperfrequency in Paris [Battaglia et al., 1957 and 1958]. While building this instrument in the Physics of the Atmosphere Laboratory of the Faculty of Science of Paris, we made a few other improvements. Other improvements or transformations are still possible (a few are in the process of being introduced).

In the microwave domain the cavities have been overdesigned. This produces a very high $Q$ factor and a broadening of the frequency band which can be utilized with each group of klystron guidecavity. In this manner we have succeeded in covering the $7-17 \mathrm{Gc} / \mathrm{s}$ range using only two microwave setups (klystrons X 13 B and X 12 Varian). Instead of klystrons, "carcinotrons" tubes of the Compagnie Générale de Télégraphie Sans Fil could be successfully used to cover the totality of the microwave band. It thus appears that it is not absolutely necessary to call on a nonresonating cavity to enlarge the frequency band.

The instrument works for gas pressures ranging from a few millimeters of mercury to $1 \mathrm{~atm}$. There is, however, no obstacle to use of higher pressures if one makes a few simple modifications to the cavities. The temperature can be varied at will since the thermostatic control of the cavities now covering an interval from 0 to $50{ }^{\circ} \mathrm{C}$ is very easily realized. 
From the viewpoint of absorption, the instrument is conceived to measure the absorption of a gas whose absorption line has a width large with respect to the response signal of the measurement cavity. But if the absorption is too strong, the cavities' responses appear too flattened and weakened. Consequently, under the present experimental setup one cannot measure absorption coefficients greater than about $30 \cdot 10^{-3}$ neper $/ \mathrm{m}$. This limitation would be of some concern in the case, rather "exceptional," of ammonia. The weakest measureable absorption (in the $\mathrm{X}$ band) would be of the order of $10^{-6}$ neper $/ \mathrm{m}$ and we have indeed measured values of $10^{-4}$ and even $10^{-5}$ neper $/ \mathrm{m}$. As far as the index of refraction is concerned, there is practically no upper limit, the lower limit being in the neighborhood of $\Delta n \approx 10^{-8}$. The precision varies from 0.03 to 2 percent according to the type and condition of the experiment. All measurements are stable and easily reproducible.

\subsection{Basic Principle}

Let us superpose on the reflecting tension of the klystron a symmetric triangular voltage (the symmetric form appears more convenient than the sawtoothed shape) and let us guide the energy produced by the klystron, which is frequency modulated, to the cavities' openings.

Let us call $\nu_{c}$ and $Q_{0}$ the resonance frequency and the quality factor of the empty cavity. If one introduces a gas into the cavity, these quantities become $\nu$ and $Q$ and according to [Goudet and Chavance, 1955; Montgomery, 1947; Jen, 1947 and 1948] $]^{2}$ we shall have:

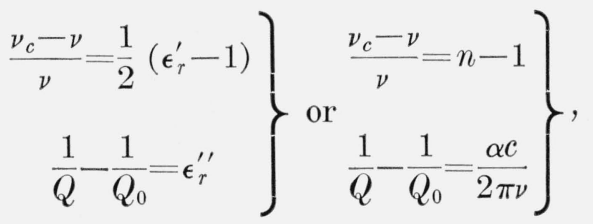

$$
\begin{aligned}
& \left(\begin{array}{c}
\epsilon_{r}^{\prime}-1=n^{2}-1 \approx 2(n-1), \\
\epsilon_{r}^{\prime \prime}=\frac{\alpha c}{2 \pi \nu}
\end{array}\right)
\end{aligned}
$$

where $\boldsymbol{\epsilon}_{r}=\boldsymbol{\epsilon}_{r}{ }^{\prime}-i \boldsymbol{\epsilon}_{r}{ }^{\prime \prime}$ is the relative dielectric constant of the gas, $m$ is its index of refraction (close to 1 ), $\alpha$ its absorption coefficient in neper $/ \mathrm{m}$ (small with respect to 1), $\nu$ and $\nu_{c}$ in $\mathrm{c} / \mathrm{s}$ and $\mathrm{c} \approx 3 \cdot 10^{8} \mathrm{~m} / \mathrm{sec}$ (the relative magnetic permeability of the gas $\mu_{r}$ is taken equal to 1 ).

The measurement of the displacement of the resonance frequency and the increase of the loss factor of the cavity following the introduction of the gas provides us with an effective means to measure $n$ and $\alpha$. The precision will depend on the method used to measure the corresponding deformation of the cavity response.

${ }^{2}$ The rationalized MKS system of units is used throughout this paper, except when otherwise specified.

\subsection{Fundamental Theory of the Instrument}

The frequency of the energy produced by the modulated klystron follows the law:

$\nu=\bar{\nu}+v t$ during a half period of modulation,

$\nu=\bar{\nu}+v \cdot(T-t)$ during the other half period,

where $T$ is the period of modulation of frequencies (1.50 sec in our case) and $v$ the velocity of modulation in $\mathrm{c} / \mathrm{s}^{2}$ (of the order of $10^{8}$ to $10^{9} \mathrm{c} / \mathrm{s}^{2}$, for example) (fig. 1a).

The microwave energy is then divided between two channels including the cavities $C_{1}$ and $C_{2}$ (fig. 2). Let us consider the first channel in which the cavity $C_{1}$ resonates at the frequency $\nu_{1}$ and possesses a factor $Q_{1}$ sufficiently high so that the incident power on the crystal detector can be written [Goudet and Chavance, 1955; Montgomery, Dijke, and Purcell, 1948]:

$$
P_{1}=P_{1}^{\max } \frac{1}{1+4 Q_{1}^{2}\left(\frac{\nu-\nu_{1}}{\nu_{1}}\right)^{2}},
$$

where $P_{1}^{\max }$ is the transmitted power at the resonance frequency $\nu_{1}$. In general, the voltage detected at the crystal terminals follows a law of the form:

$$
V_{1}=(C t e) \cdot P^{1 / \mathrm{m}}
$$

If the incident power is sufficiently weak $\left(P_{1}^{\max }<10 \mu\right.$ $W$, for example), the detection is quadratic $(m=1)$ and the detected voltage depends on the frequency according to the same law of (1.4). The detection becomes linear $(m=2)$ as soon as the power incident on the crystal approaches or becomes greater than a milliwatt. In all cases, one supposes that the detected voltage curve $V(\nu)$ presents a maximum for $\nu=\nu_{1}$ and remains symmetric around that maximum. This is the only limitation imposed on the law regulating the crystal detector.

The detected signal is then applied to the input of a linear amplifier whose output is then fed to an impulse circuit. This latter produces a short impulse (of the order of 1 to $2 \mu \mathrm{sec}$ ) when the klystron frequency reaches the value for which the output voltage of the amplifier becomes a predetermined fraction of the voltage $V^{\max }$ at resonance. The impulses are thus triggered when:

$$
\frac{V_{1}^{ \pm}}{V_{1}^{\max }}=\alpha_{1}, \quad\left(0<\alpha_{1}<1\right)
$$

and correspond to two frequencies $\nu^{ \pm}$symmetric with respect to $\nu_{1}$ :

$$
\left.\begin{array}{l}
\nu_{1}^{+}=\nu_{1}\left(1+\frac{k_{1}}{2 Q_{1}}\right) \\
\nu_{1}^{-}=\nu_{1}\left(1-\frac{k_{1}}{2 Q_{1}}\right)
\end{array}\right\},
$$




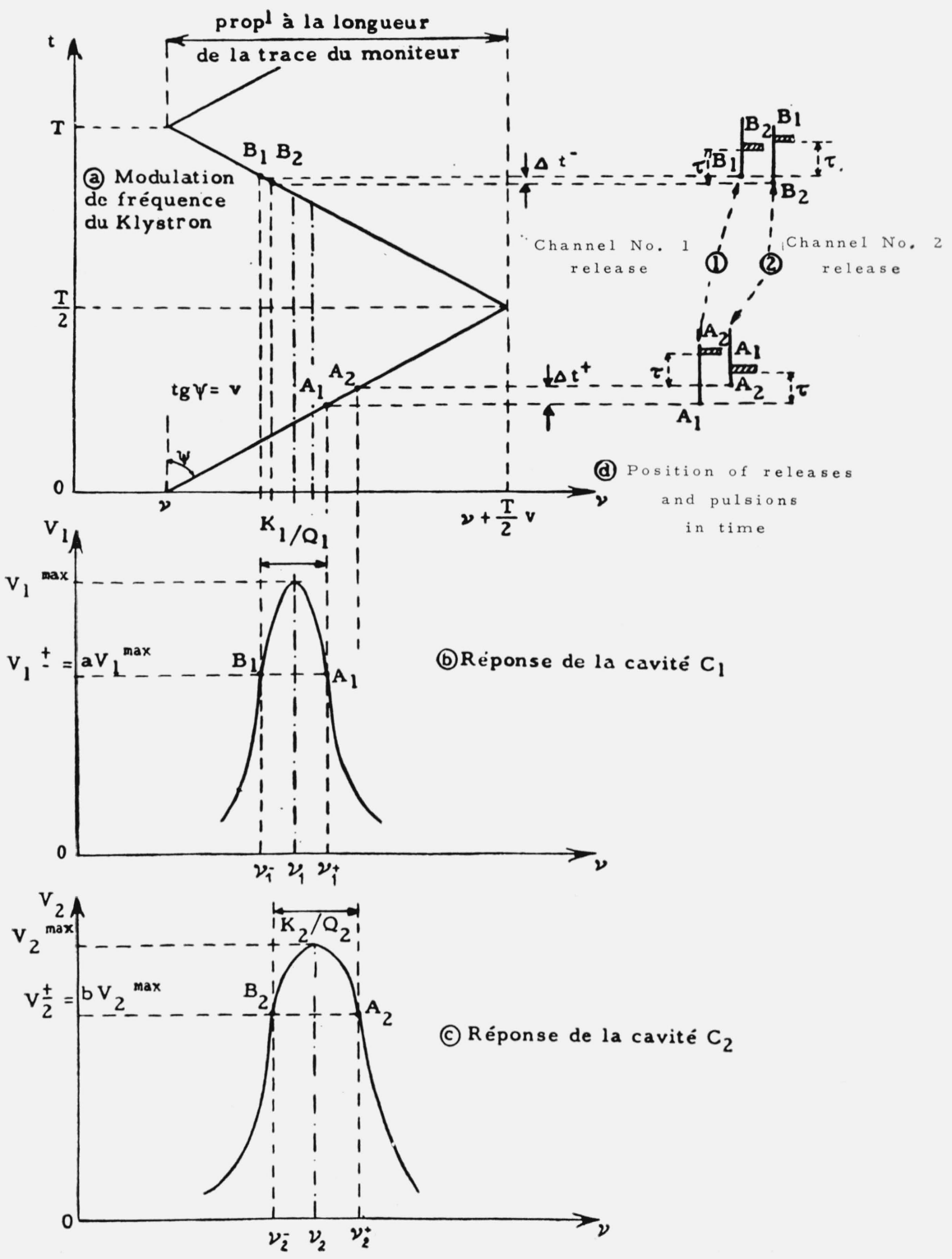

Figure 1. Operational scheme of the instrument.

$k_{1}$ being a parameter which depends on the ratio $a_{1}$ and the detection law of the crystal. One finds easily:

$$
\left.\begin{array}{l}
k_{1}=\sqrt{\left(\frac{1}{\alpha_{1}}\right)-1} \text { for a quadratic detection, } \\
k_{1}=\sqrt{\left(\frac{1}{\alpha_{1}}\right)^{2}-1} \text { for a linear detection. }
\end{array}\right\}
$$

If we require that the instrument be capable to discriminate frequencies for which (1.6) is verified, independently of any eventual variation of $V_{1}^{\max }$, it is evident that the impulse circuits must possess a memory so that they can remember the value $V_{1}^{\max }$ corresponding to each half period of modulation. The frequency $\nu_{1}^{+}$will be correctly discriminated during the half period for which $(d \nu / d t)>0$ and frequency $\nu_{1}^{-}$during the half period $(d \nu / d t)<0$.

The first channel produces during each period of the modulation voltage two well-defined impulses, the first one appears at the point $A_{1}$ and the second at the point $B_{1}$ of the response curve of the cavity (fig. 1b). The second channel, in turn, produces two similar impulses whose forward fronts correspond to the times at which the klystron passes through the frequencies:

and

$$
\left.\begin{array}{l}
\nu_{2}^{+}=\nu_{2}\left(1+\frac{k_{2}}{2 Q_{2}}\right), \text { when } d \nu / d t>0 \\
\nu_{2}^{-}=\nu_{2}\left(1-\frac{k_{2}}{2 Q_{2}}\right), \text { when } d \nu / d t<0
\end{array}\right\}
$$




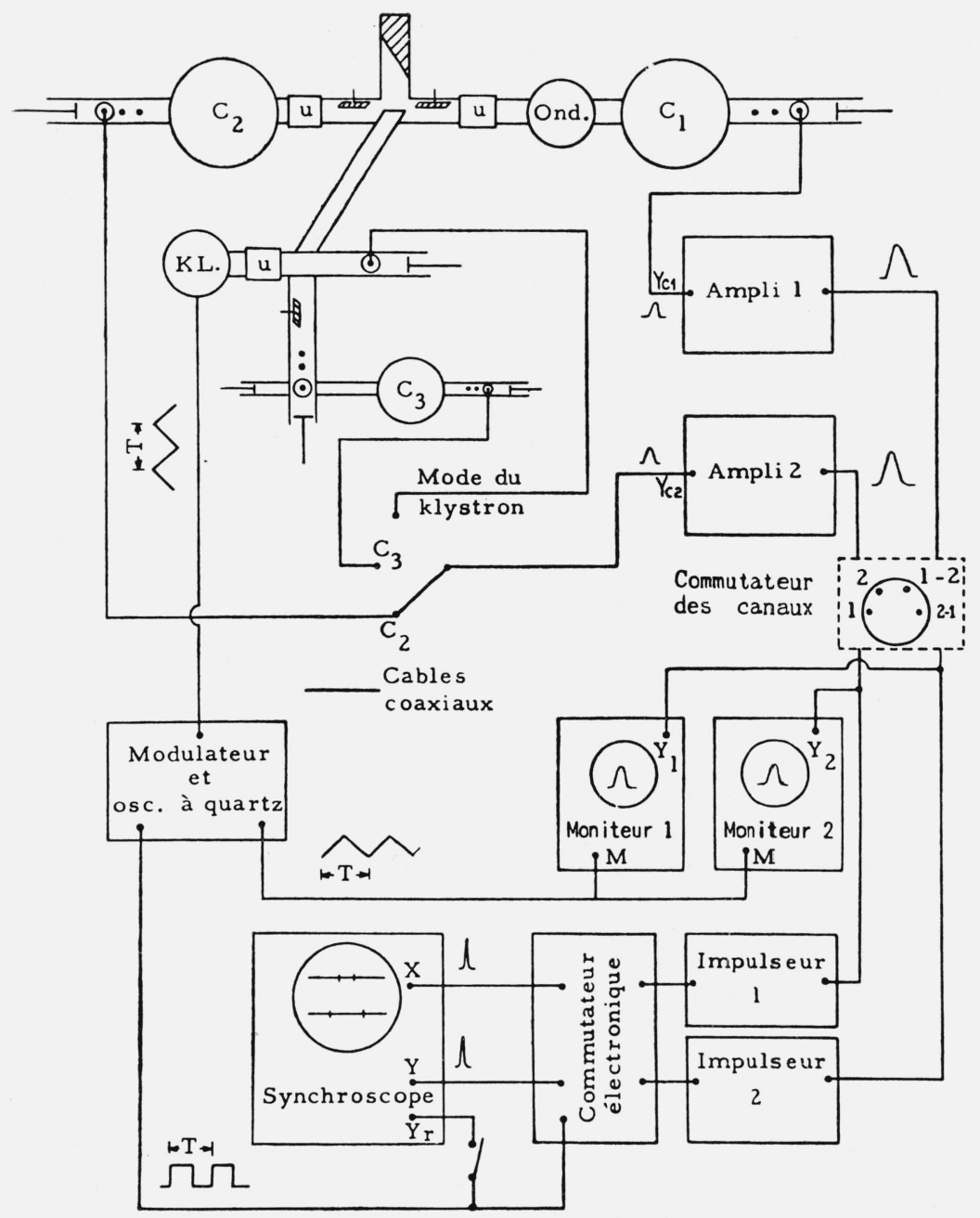

Figure 2. Bloc diagram of the instrument.

$k_{2}$ depends on the detection law of the crystal and on the predetermined parameter:

$$
a_{2}=\frac{V_{2}^{ \pm}}{V_{2}^{\max }},\left(0<a_{2}<1\right)
$$

and $\nu_{2}$ is the resonance frequency of the cavity $C_{2}$ (fig. 1c). To keep the argument more general, one admits that $Q_{1} \neq Q_{2}$ and $k_{1} \neq k_{2}$. Equation (1.8) remains the same with the substitution of $a_{2}$ and $k_{2}$ for $a_{1}$ and $k_{1}$, respectively.

We utilize the pulses produced by the first channel, say, to fire the synchroscope sweep and the pulses from the second channel, retarded by an amount of time $\tau$, which are then applied to the plates fixing the vertical deviation of the synchroscope (see fig. 1d). On these same plates we superimpose a rectangular signal of duration $T / 2$, synchronized with the modulation voltage of the klystron. One then observes on the screen of the synchroscope two parallel traces, each corresponding to a half period of modulation, and on each trace a pulse will appear (fig. 3).

Following (1.3), if $\Delta \nu$ is the difference between the frequencies produced by the two generators during a half period of the modulation voltage, this difference of frequencies corresponds to a time separation of:

$$
\Delta t=\frac{\Delta \nu}{v}
$$

Consequently, the distances $d_{1}^{+}$and $d_{1}^{-}$reckoned from the origin of the sweep of the two pulses as well as the separation $d_{1}$ between the two pulses (fig. 3) are expressed by the relations:

$$
\left.\begin{array}{l}
d_{1}^{+}=\tau+(\Delta t)^{+}=\tau+\frac{\nu_{2}-\nu_{1}}{v}+\frac{1}{2 v}\left(\frac{k_{2} \nu_{2}}{Q_{2}}-\frac{k_{1} \nu_{1}}{Q_{1}}\right), \\
d_{1}^{-}=\tau+(\Delta t)^{-}=\tau-\frac{\nu_{2}-\nu_{1}}{v}+\frac{1}{2 v}\left(\frac{k_{2} \nu_{2}}{Q_{2}}-\frac{k_{1} \nu_{1}}{Q_{1}}\right), \\
d_{1}=\left|d_{1}^{+}-d_{1}^{-}\right|=\left|\frac{2}{v}\left(\nu_{2}-\nu_{1}\right)\right| .
\end{array}\right\}
$$

These distances are measured in seconds and for that reason the screen is calibrated in $\mu \mathrm{sec} / \mathrm{cm}$.

One notes that the separation $d_{1}$ between the two 

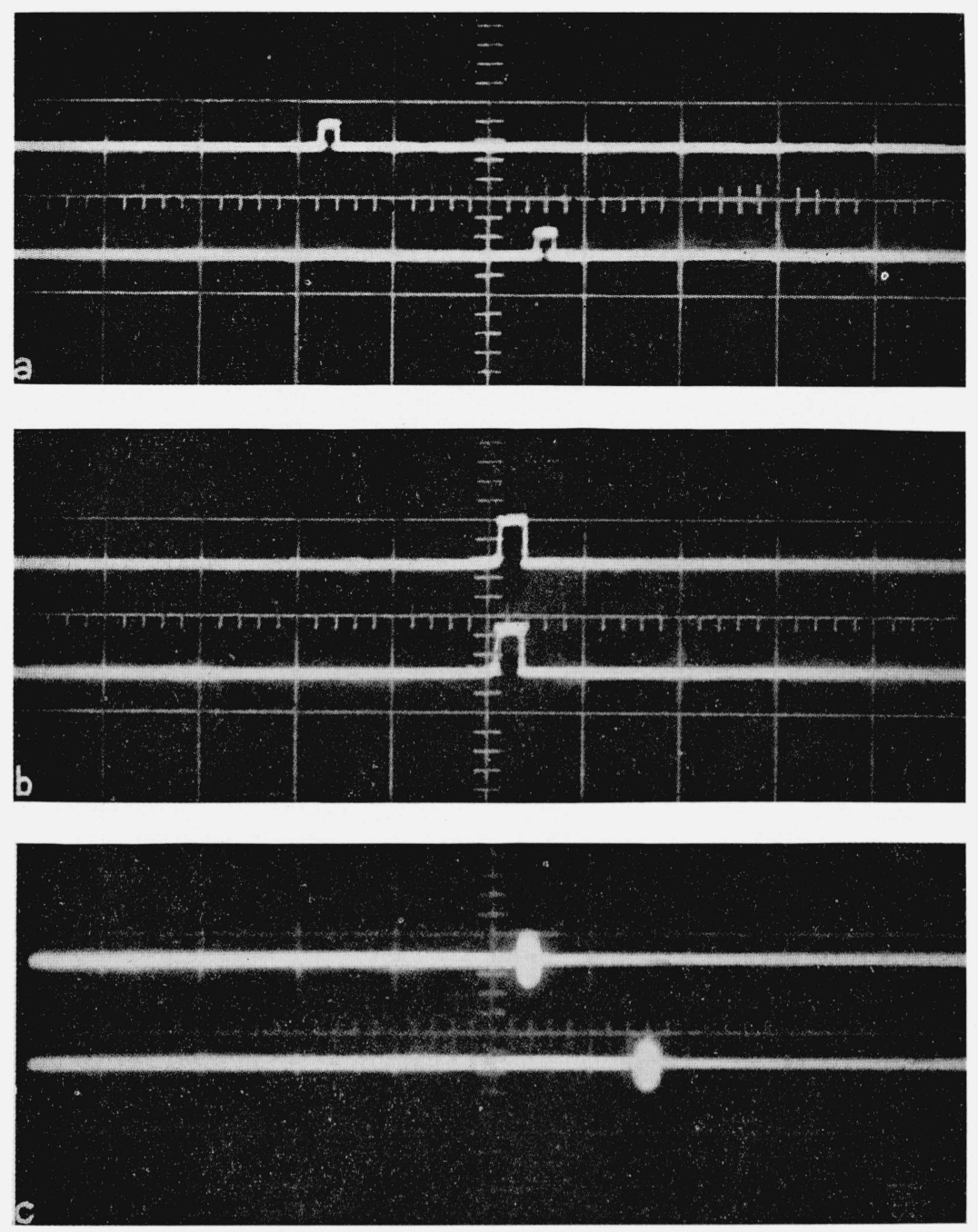

Figure 3. Pictures of two pulses on the screen of the synchroscope:

(a) Pulses normally applied to the plates controlling the vertical deviation of the oscilloscope (scale of $10 \mathrm{microseconds} / \mathrm{cm}$;

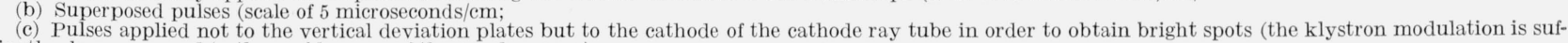
ficiently slow compared to the rapid sweep of the synchroscope).

pulses is directly proportional to the difference between the resonance frequencies of the two cavities. The two pulses will be superimposed, i.e., exactly alined vertically on the screen, only when the two cavities have the same resonance frequencies $\left(\nu_{2}=\right.$ $\left.\nu_{1}=\nu_{c}\right)$.

Let us suppose that at the beginning of the experiment the two cavities have been evacuated and tuned to the same frequency $\nu_{c}$ (superimposed pulses). We introduce then in the measuring cavity, $C_{2}$, gas at a given pressure $p$. The other cavity, $C_{1}$, is still empty and is used as a frequency standard. The resonance frequency of $C_{2}$ is shifted to the new frequency $\nu_{c} / n$ where $n$ is the index of refraction of the gas at pressure $p$ and at the fixed temperature of the cavity $C_{2}$. The pulses will now appear on the screen separated by a distance $d_{1}$ given by the relation:

$$
d_{1}=\left|\frac{2 v_{c}}{v}\left(\frac{1}{n}-1\right)\right| .
$$

If one measures $d_{1}$ in $\mu \mathrm{sec}, \nu$ in $\mathrm{Mc} / \mathrm{s}$ and $v$ in $\mathrm{Mc} / \mathrm{s}^{2}$, we shall have, since the index of refraction is always close to unity:

$$
N=\left(n_{o}-1\right) \cdot 10^{6}=\frac{v}{2 \nu_{c}} d_{1}
$$

It is evident that no principle requires the superposition of the pulses at the beginning of the experiment since the index can generally be measured by the variation of the separation between the pulses 
caused by the introduction of the gas into one of the cavities.

The only parameter that does enter the measurement of the index of refraction is $v$, the instrument constant. The $k$ parameters, the eventual differences between the $Q$ of the cavities and the detection laws of the crystals do not come in at all into the relations.

The principal advantage of this technique resides in the fact that the pulses furnish a precise indication of the relative position of the response curves of the cavities while the comparison between the responses is made in a very short interval of time, even nil if the pulses are superimposed. This for all practical purposes eliminates the effects of possible fluctuation in the frequency or power of the klystron in time.

Let us examine now the effect that the gas absorption, if it exists, will have on the positions of the two pulses. This effect will be a change in the position of the barycenter of the two pulses whose distance from the origin of the sweep is given by:

$$
d_{1 \mathrm{bar}}=\frac{1}{2}\left(d_{1}^{+}+d_{1}^{-}\right)=\tau+\frac{1}{2 v}\left(\frac{k_{2} \nu_{2}}{Q_{2}}-\frac{k_{1} \nu_{1}}{Q_{1}}\right) .
$$

When the two cavities are tuned to the same frequency $\nu_{1}=\nu_{2}=\nu_{c}$ the distance of the two superimposed pulses from the origin of the sweep is:

$$
d_{1 \mathrm{bar}}^{0}=\tau+\frac{\nu_{c}}{2 v}\left(\frac{k_{2}}{Q_{2}}-\frac{k_{1}}{Q_{1}}\right) .
$$

If we introduce gas into the measuring cavity, the two pulses will shift position with respect to each other. They can be brought back together, however, if one retunes the measuring cavity. Then once more the two pulses are superposed but at a new distance from the origin of the sweep given by:

$$
d_{1 \mathrm{bar}}^{\mathrm{gas}}=\tau\left(+\frac{\nu_{c}}{2 v} \frac{k_{2}}{Q_{2}^{\text {gas }}}-\frac{k_{1}}{Q_{1}}\right) .
$$

$Q_{2}^{\text {aas }}$ is the quality factor of the measuring cavity when it is filled with gas. We deduce that:

$$
\Delta d_{1 \mathrm{bar}}=d_{1 \mathrm{bar}}^{\mathrm{gas}}-d_{1 \mathrm{bar}}^{0}=\frac{k_{2} \nu_{c}}{2 v}\left(\frac{1}{Q_{2}^{\mathrm{gas}}}-\frac{1}{Q_{2}}\right),
$$

and recalling (1.2) we finally obtain:

$$
\text { coefficient of absorption } \alpha=\frac{4 \pi v}{k_{2} \cdot c} \cdot\left(\Delta d_{1 \text { bar }}\right) \text {. }
$$

The coefficient of absorption is thus directly proportional to the displacement of the barycenter of the pulses. The superposition of the pulses is not a requirement since the barycenter can be determined from the half sum appearing in (1.17). But this method of superposition has the advantages, among others, to give the absorption for a well-determined frequency $\nu_{c}$.
Absorption measurements require not only the modulation velocity $v$ but also the parameter $k$ whose absolute determination necessitates the knowledge of the law of detection of the crystal. We shall return later to the questions of calibrations.

\subsection{Absorption Measurements by Electronic Com- mutation of Channels}

The preceding analysis supposes that the pulses coming out from one of the two channels are constantly utilized to start the oscilloscope's sweep, while the pulses from the other channel are applied on the plates providing vertical deviation. Let us incorporate now into the pulse generator circuits, an electronic commutator which will insure the permutation of channels every period $T$ of modulation of the klystron. One will then observe four pulses on the screen of the oscilloscope, two of them the same as before, since they are started by channel $C_{1}$, the others being generated by the other channel (fig. 1d). Figure 4 shows a picture of the oscilloscope screen.

Equations (1.12) and (1.13) continue to express the distances from the origin of the sweep for the two pulses which correspond to modulation periods during which the firing is assured through channel $C_{1}$. To other periods correspond two other pulses whose distances from the origin are

$$
d_{2}^{+}=\tau-\Delta t^{+}=\tau-\frac{\nu_{2}^{+}-\nu_{1}^{+}}{v}=\tau-\frac{\nu_{2}-\nu_{1}}{v}-\frac{1}{2 v}\left(\frac{k_{2} \nu_{2}}{Q_{2}}-\frac{k_{1} \nu_{1}}{Q_{1}}\right)
$$

$$
d_{2}^{-}=\tau-\Delta t^{-}=\tau+\frac{\nu_{2}^{-}-\nu_{1}^{-}}{v}=\tau+\frac{\nu_{2}-\nu_{1}}{v}-\frac{1}{2 v}\left(\frac{k_{2} \nu_{2}}{Q_{2}}-\frac{k_{1} \nu_{1}}{Q_{1}}\right) .
$$

The two pulses of the upper traces and the two pulses of the lower traces are distance from each other by amount:

$\left.\begin{array}{l}d^{+}=\left|d_{1}^{+}-d_{2}^{+}\right|=\left|2 \frac{\nu_{2}-\nu_{1}}{v}+\frac{1}{v}\left(\frac{k_{2} \nu_{2}}{Q_{2}}-\frac{k_{1} \nu_{1}}{Q_{1}}\right)\right|, \\ d^{-}=\left|d_{1}^{-}-d_{2}^{-}\right|=\left|-2 \frac{\nu_{2}-\nu_{1}}{v}+\frac{1}{v}\left(\frac{k_{2} \nu_{2}}{Q_{2}}-\frac{k_{1} \nu_{1}}{Q_{1}}\right)\right| \cdot\end{array}\right\}$

One easily verifies the following propositions:

(1) The four pulses will, in general, form, at least for the condition under which we operate, an isosceles trapeze (fig. 4).

(2) If one superimposes either the two pulses on the upper traces $\left(d^{+}=0\right)$ or the two pulses on the lower trace $\left(d^{-}=0\right)$, the pulses on the other trace are distant from each other (within the valid approximation $\left(k_{2} / Q_{2}<<2\right)$ by an amount $d$ given by:

$$
d=\left|\frac{2 \nu}{v}\left(\frac{k_{2}}{Q_{2}}-\frac{k_{1}}{Q_{1}}\right)\right|, \quad\left(\nu \approx \nu_{1} \approx \nu_{2}\right) .
$$

(3) The pulses will superimpose on each other two by two on the upper trace and the lower trace simul- 

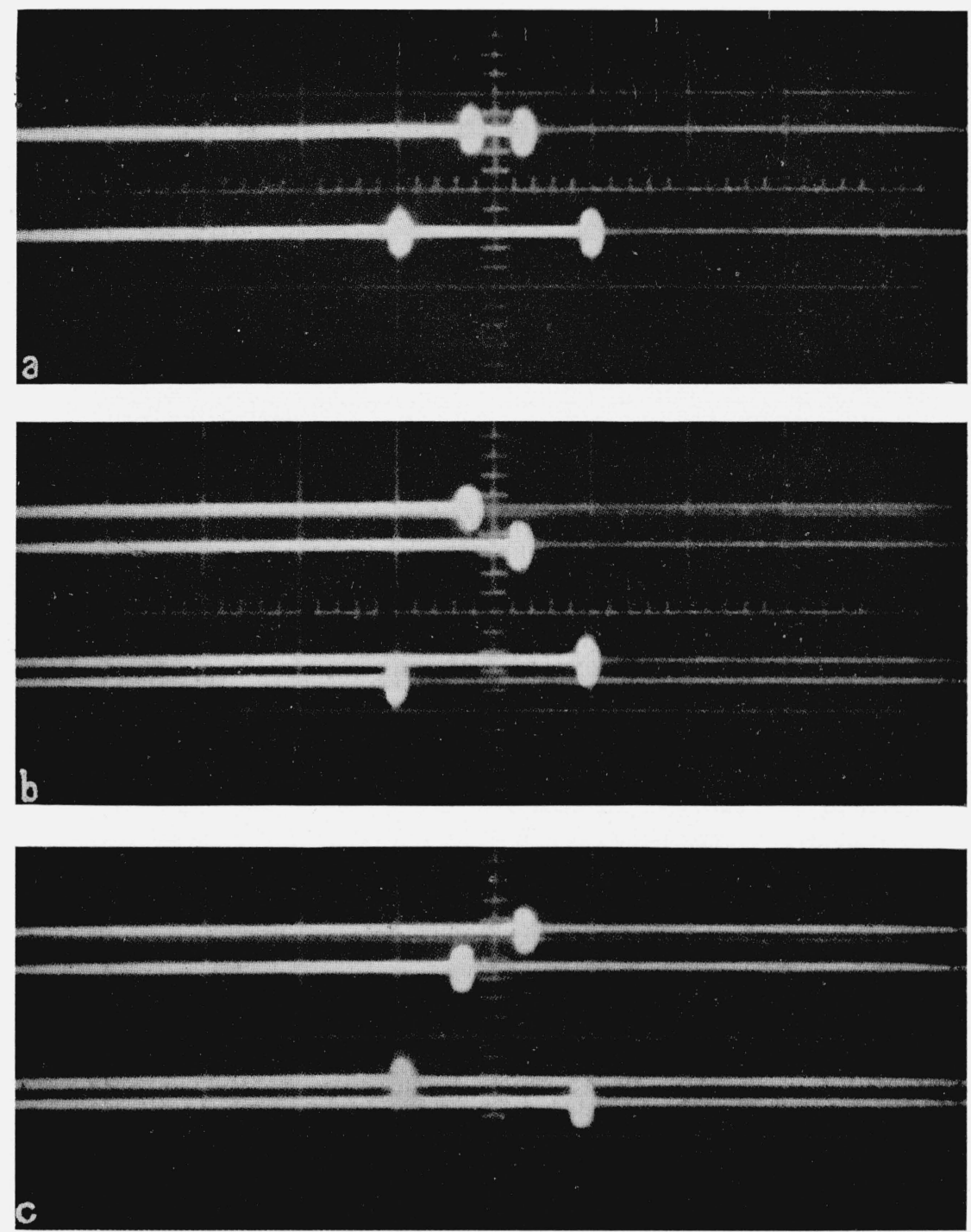

FIgURe 4. Pictures of the four pulses on the synchroscope screen (pulses applied by the cathode of the cathode ray tube, electronic

(a) Without labeling of the pulses; commutation of the operating channels:

b) With labeling of the pulses and with the manual inverting switch turned to one or the other of its two positions;

(c) With labeling of the pulses and with the manual inverting switch turned to one or the other of its two positions.

taneously $\left(d^{+}=0\right.$ at the same time that $\left.d^{-}=0\right)$ only if the two following conditions are fulfilled:

$$
\nu_{1}=\nu_{2} \text { and } \frac{k_{1}}{Q_{1}}=\frac{k_{2}}{Q_{2}}
$$

In order to arrive at formulas which are easily applicable in practice, we make a previous adjustment of the instrument to insure that the simplifying condition $k_{1}=k_{2}=k$ is permanently fulfilled. To assure this, it is sufficient to send the response signal of one cavity to the input terminal of the two pulse generators. This will force these two generators to operate on the same response curve; that is with $Q_{1}=Q_{2}$ and $\nu_{1}=\nu_{2}$. By adjusting the height discrimination $a_{1}$ and $a_{2}((1.6)$ and $(1.10))$ so as to superim- pose not only the pulses of the upper trace but also those of the lower trace, one then will have following $(1.27)$

$$
k_{1}=k_{2}=k .
$$

Then (1.26) provides a simple means for absorption measurement. During a first run, the cavity being empty, one superimposes the pulses on the upper trace, for instance, and one measures the spread between the pulses on the other trace, given by (1.26) with $k_{1}=k_{2}=k$. When during a second run, one introduces a sample of gas into the measurement cavity and one retunes the cavity so that the two pulses of the upper trace are once more superimposed, the distance between the two lower pulses becomes:

$$
d_{\mathrm{gas}}=\left|\frac{2 \nu k}{v}\left(\frac{1}{Q_{2}^{\mathrm{gas}}}-\frac{1}{Q_{1}}\right)\right| .
$$


Let us take the difference:

$$
\Delta d=d_{\mathrm{gas}}-d=\frac{2 v k}{v}\left(\frac{1}{Q_{2}^{\mathrm{gas}}}-\frac{1}{Q_{2}}\right) .
$$

We finally obtain taking into account (1.2):

$$
\text { coefficient of absorption } \alpha=\frac{\pi v}{k c} \cdot(\Delta d) \text {, }
$$

which formula can be applied without modification to mixed units : $v$ in $\mathrm{Mc} / \mathrm{s}^{2}, d$ in $\mu \mathrm{sec}, c$ always equal to $3 \cdot 10^{8} \mathrm{~m} / \mathrm{sec}$ and $\alpha$ in neper $/ \mathrm{m}$.

This last equation indicates through comparison with (1.21) that the sensitivity of the instrument has been increased by a factor of 4 thanks to the technique of channel commutation.

For absorption measurements it is convenient although not necessary to also realize the initial condition $Q_{2}=Q_{1}$. To do this we must first decide which of the two cavities has the higher $Q$ factor. This cavity will be selected as the reference one. To reduce its factor of quality to that of the other cavity one must introduce into the reference cavity an absorbing gas under an appropriate pressure (for instance, argon mixed with a bit of ammonia). After adjusting as before, so that $k_{1}=k_{2}=k$, one reestablishes normal connection between the two cavities and the two pulse generator assemblies and one adjusts the pressure in the reference cavity, the other cavity being empty, so that the upper and lower pulse once more are superimposed. Following (1.27), the supplementary condition $Q_{2}=Q_{1}$ has now been filled.

This four pulse technique is of no particular interest for measurement of the index of refraction. The index can be measured at the same time as the absorption, however, if one uses a commutator that can suppress at will the electronic commutation of the channels. The simultaneous measurement of the index (sec. 3) becomes easy then.

\subsection{Differential Measurement of Dispersion}

The study of dispersion at twice the frequency can be accomplished in the following fashion: Take a third channel $C_{3}$ (fig. 2) identical to the other two but including a cavity $C_{3}$ which resonates at $2 \nu$, twice the frequency of the other cavities and excited by the same klystron through a crystal which doubles the frequency.

Let us, for instance, use the signals coming from this cavity and from cavity $C_{1}$. If one supposes that the gas is introduced only in one of these cavities, the other being empty, one obtains the same results, (1.16) and (1.21), provided that the measured quantities pertain either to the frequency $\nu_{c}$ (gas in $C_{1}$ ) or to the double frequency $2 \nu_{c}$ (gas in $C_{3}$ ). In the latter case, the factor 4 in the absorption formula (see (1.21)) must be replaced by 8 .

Let us suppose now that the gas is introduced simultaneously in both cavities $C_{1}$ and $C_{3}$ under the same pressure and the same temperature. One can easily show that the separation $d_{1}$ between pulses initially superimposed is proportional to the difference between the values of index of refraction at frequencies $\nu_{c}$ and $2 \nu_{c}$

$$
\left|n_{1}-n_{2}\right|=\frac{v}{2 \nu_{c}} d_{1}
$$

The introduction of a third channel allows not only for the measurement of the index of refraction and of the absorption at twice the frequency, but also the precise measurement of the possible variation of the index with frequency even if this variation is very small. One notices that in (1.31) it is the difference of indices and not their absolute values which appears.

It is also possible to measure dispersion at nearby irequencies. To that effect one impresses on the klystron a second modulation by applying on its reflector a voltage of radio frequency of a few Mc/s. We shall mention again this radio frequency modulation, but we will not dwell here on dispersion measurements at nearby frequencies [Battaglia et al., 1957 and 1958].

\subsection{Spectrometer Design}

A schematic drawing of the instrument is presented on figure 2 , and also a photograph of the actual instrument is shown (see sec. 1.10).

The microwave section of the instrument (klystron, microwave circuits, cavities) has been designed to operate in the frequency band of its klystron. Another assembly must be substituted if one wants to work in another frequency band. We have built, so far, two assemblies: One for the band from 7 to $12 \mathrm{Gc} / \mathrm{s}$ (klystrons X $13 \mathrm{~B}$ or $723 \mathrm{~A} / \mathrm{B}$ ), the other for the frequency band 13 to $17 \mathrm{Gc} / \mathrm{s}$ (klystrons X 12).

On the other hand, the electronic circuitry from the input terminals of the amplitiers to the oscilloscope operates only on signals of low frequency and remains, of course, the same for all higher frequency bands. In the following paragraphs we give a short description of the different parts of the instrument as well as a few words on their operation.

A third part of the instrument (not indicated on the drawing of fig. 2) is made up principally of a rotary vacuum pump which can be used in conjunction with a mercury vapor distillation pump, glass lines, valves, and tanks to handle gases as well as a mercury barometer. We shall not go into detail on the high vacuum techniques used although they are very important in practice.

\subsection{Microwave Section of the Instrument}

A uniline decouples the klystron (X 13 B) from the circuits into which it feeds energy. This kind of decoupling is good enough (fig. 5). A "magic 'T" metes the klystron's energy between the two channels which includes cavities $C_{1}$ and $C_{2}$. The cavities are decoupled from the source by means of dampers and 


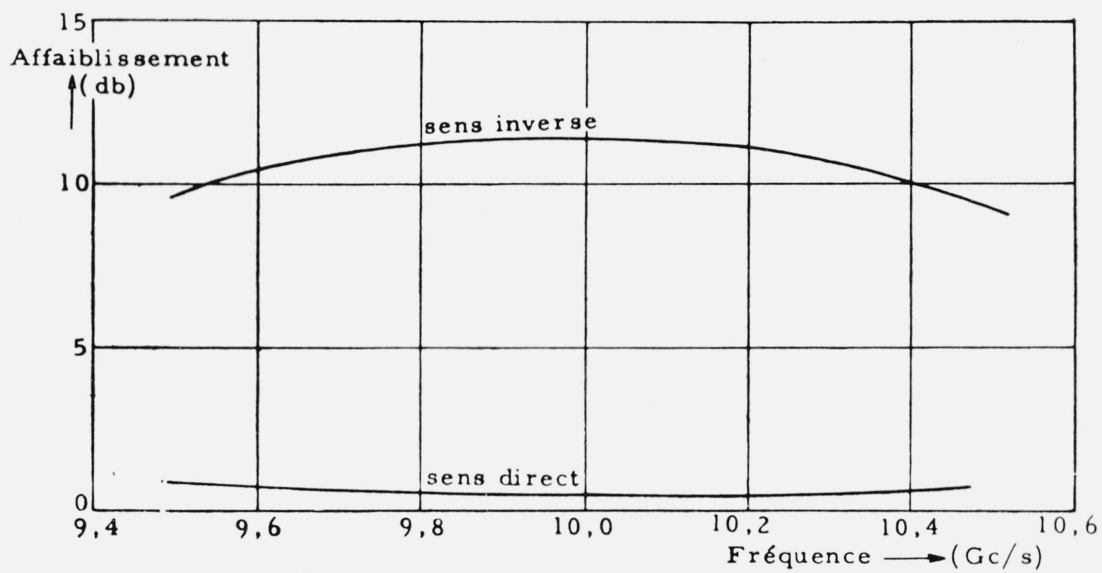

Figure 5. Attenuation curves for a unidirectional line (manufactured by L.T.T. Type DL. H 09.A. No. .008, T.O.S.1.05).

Each unit of a series production has its own attenuation curves.

unilines. This eliminates the interaction between the two channels of the instrument. A wavemeter inserted in the waveguides of the reference channel measures the wavelength of the hyperfrequency energy. In the back of the cavity, at the ends of the channel, the signals detected by crystals are carried away through coaxial cables. The instrument is tuned by means of screws and pistons.

The cavities constitute the most delicate part of this assembly. They must be carefully built so as to be well-protected against mechanical deformation following variations of the gas pressure. Their design must be such that their quality factor $Q$ is as high as possible.

Figure 6 shows the design of our 3 -cm cavities which have been built by the Laboratoires Derveaux. The cavities are overdesigned $(80 \mathrm{~mm}$ inside diameter) and consequently the $Q$ factor is high ( $Q$ of about 35,000 ) and the frequency band large. Protection against the mechanical deformation is provided by a second chamber which receives the gas and which communicates with the cavity proper while completely surrounding it. In this fashion the walls of the principal cavity are not subject to pressure differentials. The motion of a piston insures tuning. The excitation is produced through holes of $5 \mathrm{~mm}$ diam which can be closed with mica windows when we want to evacuate the system. A telescopic system at the entrance and exit of the cavity which has been simplified as much as possible, allows for easy coupling or decoupling of the cavity and is the waveguide circuit.

The $2 \mathrm{~cm}$ cavities are slightly different. They too are overdesigned (inside diameter of $60 \mathrm{~mm}, 4 \mathrm{~mm}$ excitation holes) but thanks to a careful construction (accomplished by Mr. LeBrun) and the application of a new coupling method suggested by Bleaney-Loubser-Penrose [1947], only the mode $\mathrm{TE}_{01 n}$ can be excited and transmitted (fig. 7).

Finally the cavities are installed inside a thermostatically controlled chamber. The thermostat, shown on the photograph allows us to vary the

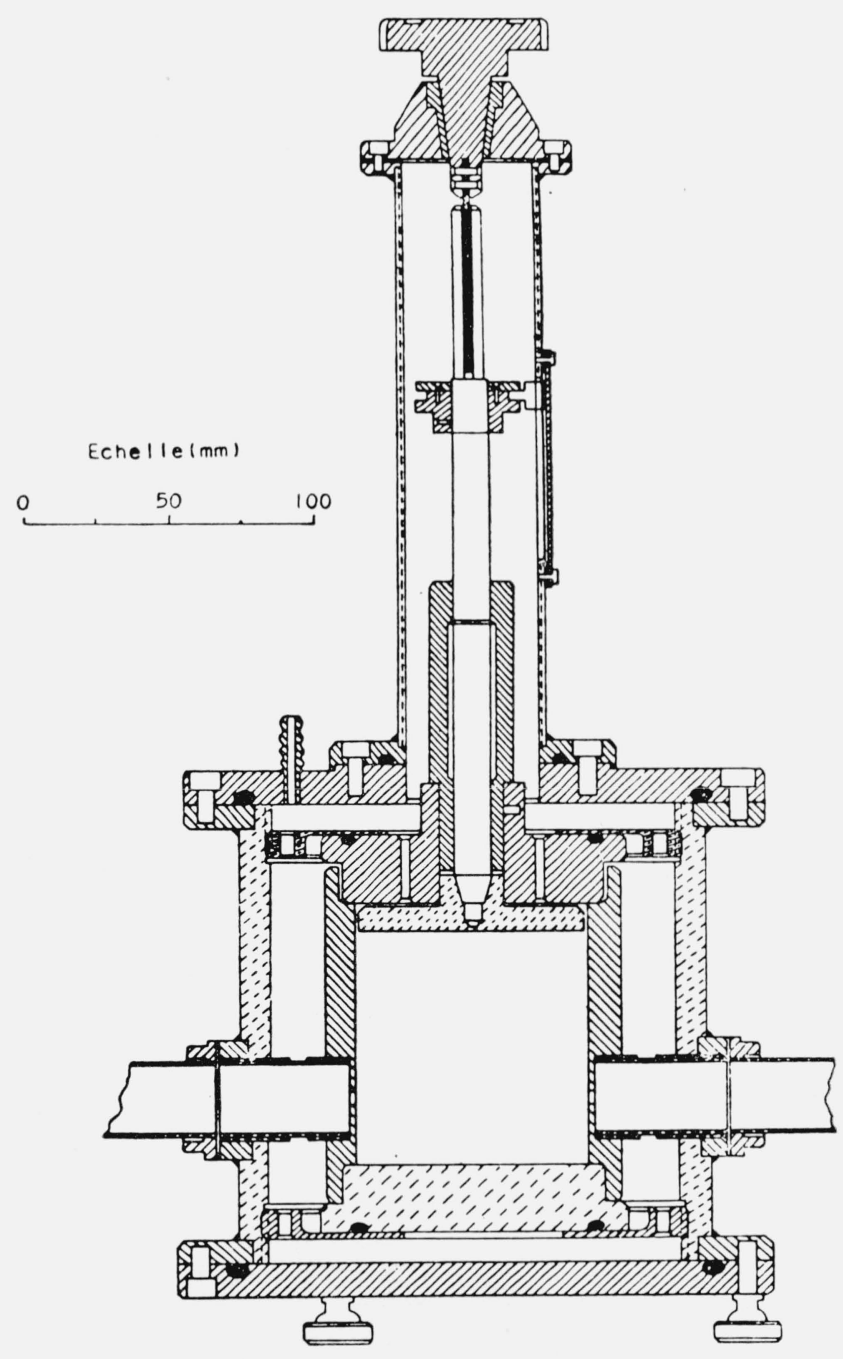

Figure 6. Drawing of a "3-cm" cavity. 


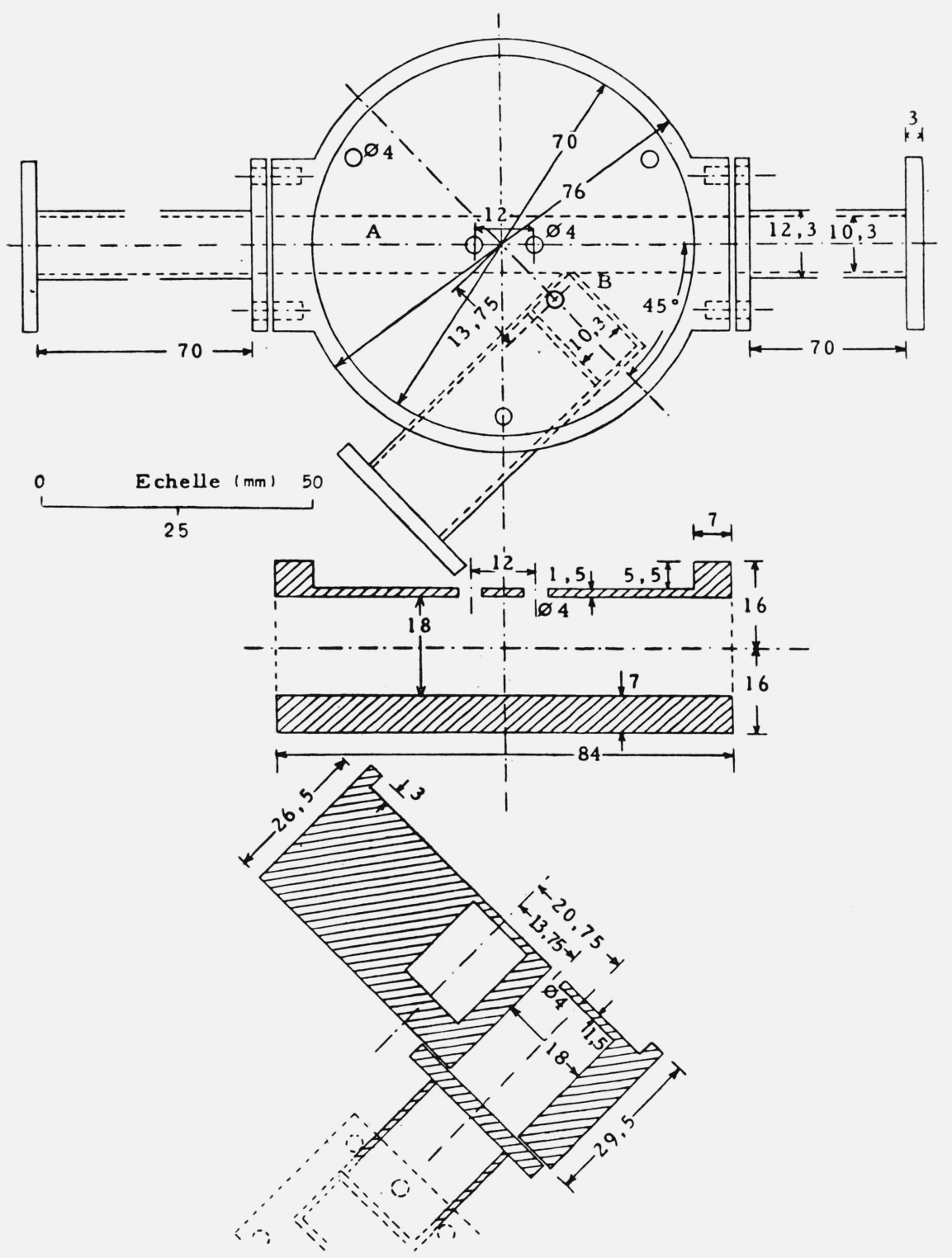

FIGURE 7. Drawing of the lower part of a "2-cm" cavity.

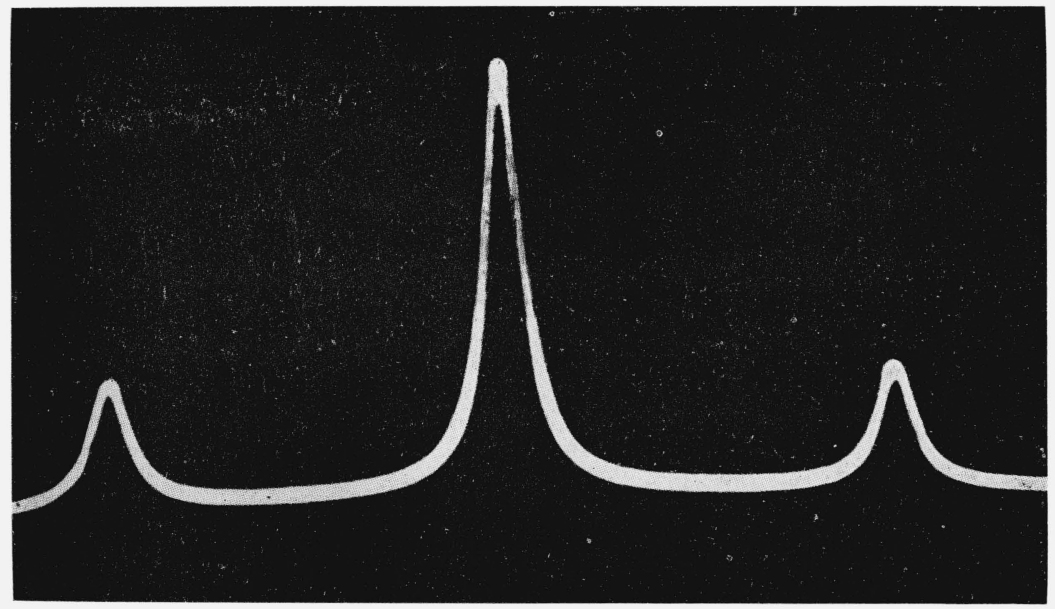

FIGURE 8. Response of a cavity when a radiofrequency modulation is superimposed on the triangular modulation of the klystron. 
temperature from 0 to $50{ }^{\circ} \mathrm{C}$ with a precision of $0.1{ }^{\circ} \mathrm{C}$.

The $Q$ factor of the cavity is measured in the following fashion. We impose on the klystron a second modulation frequency by applying on its reflector a voltage of radio frequency $f$ (from 1 to a few $\mathrm{Mc} / \mathrm{s}$ ). The microwave energy is then distributed in the several lateral bands $\nu \pm n f$, but one can adjust the depth of this second modulation so that the greater part of its energy is concentrated in the first bands $\nu \pm f$. Under those conditions the cavity transmits a response of the form shown on figure 8. The distance in frequency unit between the two lateral resonances being known $(=2 f)$, the horizontal sweep is thus standardized in frequency. One can then measure in frequency units the half width of the principal resonance of the cavity and compute its $Q$ factor. A more precise measurement of $Q$ is possible if one wants to use techniques involving pulses from the totality of the instrument, but such precise knowledge of $Q$ is not necessary.

Klystron X 13 B and X 12 are cooled by water circulating inside bronze chambers which are in contact with the klystron on the reflector side and the cathode side.

\subsection{Electronic Circuitry}

The electronic circuitry is made up of the following sections:

A. Two amplifiers (one for each channel) (fig. 9)The amplifiers have been designed for large total gain (of the order of $100 \mathrm{db}$ ), for low background noise (signal-to-noise ratio of the order of $40 \mathrm{db}$ ) and for a sufficiently large band pass (30 to $20,000 \mathrm{c} / \mathrm{s}$ ).

B. Two monitors (fig. 10), which are used to flash the amplified responses from the cavities.

C. Modulators (fig. 11) - The triangular generator and the oscillator for radio frequencies are incorporated on the same chasis. The shape of the triangular signal is satisfying for its symmetry and linearity (fig. 12).
D. Pulse circuits (fig. 13)-These circuits are the most important part of the electronic circuitry. They include two discriminators, two impulse generators, one electronic commutator, and a circuit to record the pulses.

1. Discriminators - Each discriminator is made up of three tubes $\left(V_{1}, V_{2}, V_{3}\right)$. The signal from the amplifier is sent by the cathode follower $V_{1}$ to the cathode of a thyratron $V_{3}$. This same signal charges the condenser $C_{1}$ through diode $V_{2}$. The plate of thyratron $V_{3}$ is thus brought to the maximum value of the signal while the grid is polarized at a predetermined fraction (see (1.6)) of this voltage. Once the klystron has gone through the resonance frequency of the corresponding cavity, the plate and the grid of the thyratron keep their polarization, the time constant $R_{1} C_{1}$ being sufficiently high, while the voltage of the cathode which follows the response signal, decreases. The thyratron will fire when the cathode voltage reaches the value $a V_{\max }$ of the grid. This process repeats itself every half period so that an eventual variation of the $V_{\max }$ has no appreciable influence on $a$ (electronic memory parameter). The voltage divider allows one to choose the appropriate value of $a=3 / 4$ or $1 / 2$.

2. Electronic commutator for the channels-This circuit which we have improved works in the following fashion:

During one period of the triangular voltage which frequency modulates the klystron, thyratrons $V_{9}$ and $V_{10}$ can be fired by pulses coming from the discriminating circuits; during the following period they cannot be fired since their cathodes have received a strong positive polarization. Thyratrons $V_{8}$ and $V_{11}$ work in the same fashion but the polarizing voltage is obtained through the flip-flop $V_{5}$ which is activated by two rectangular signals coming from the modulator. Their period is thus doubled. When one of the thyratrons fires, the artificial line connected to its plate discharges, and a narrow pulse is obtained on the resistance $R_{L}$.

On each of the two $R_{L}$ resistors, one registers,

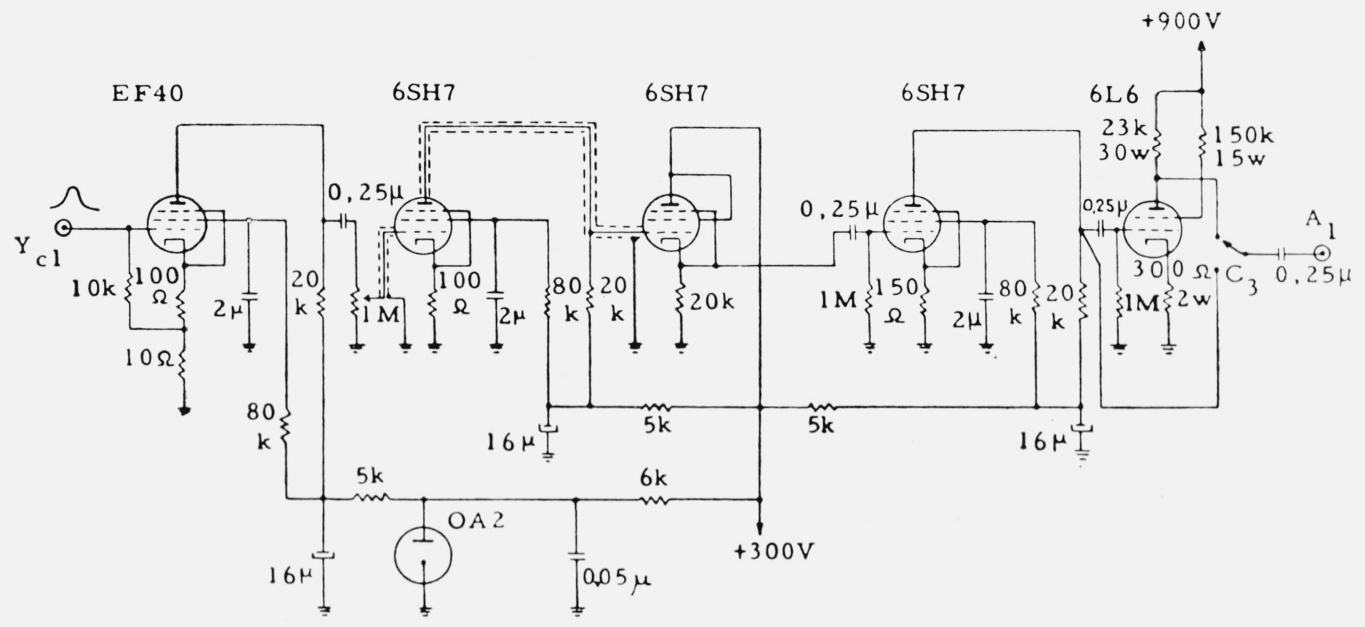

Figure 9. Diagram of one of the amplifier. 


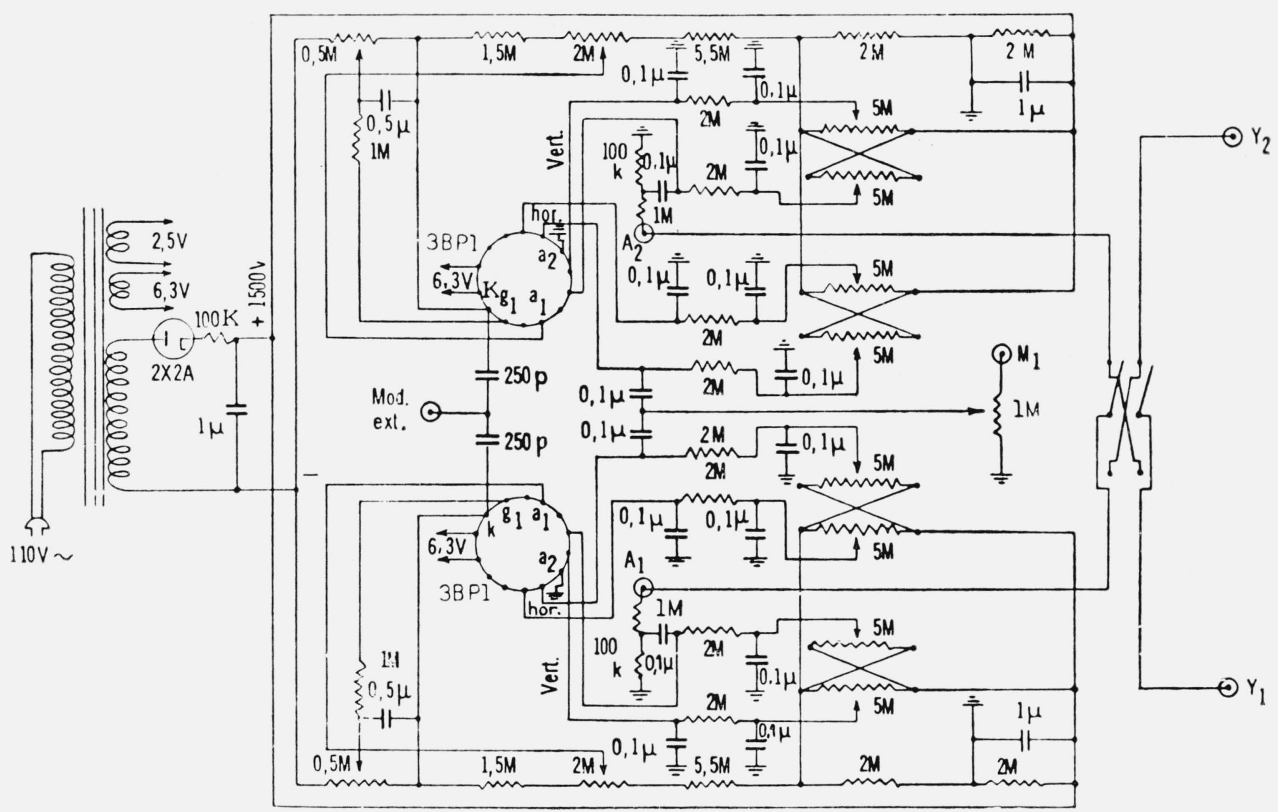

Figure 10. Diagram of one of the monitors.

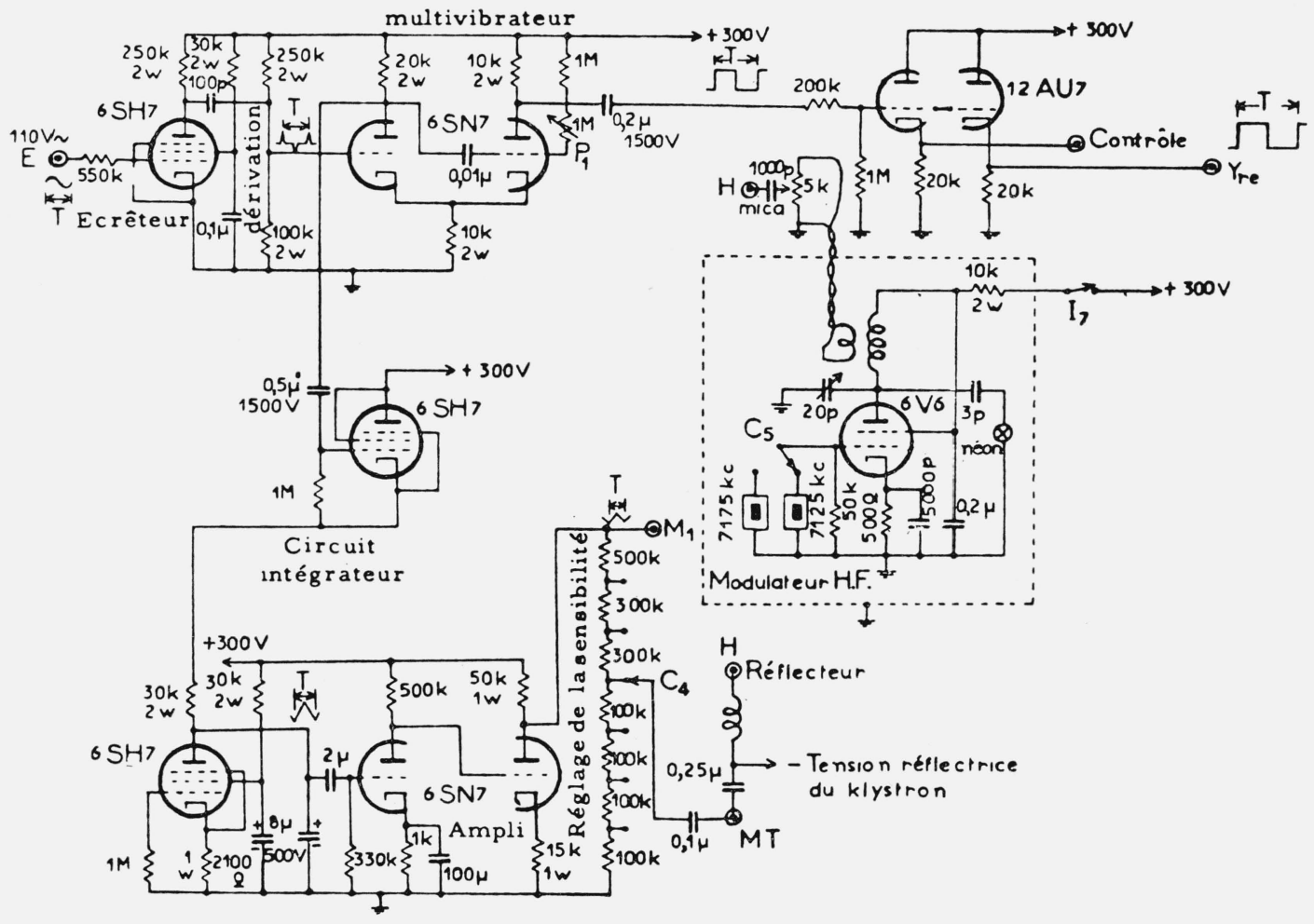

Figure 11. Diagram of the modulators (triangular and H.F.).

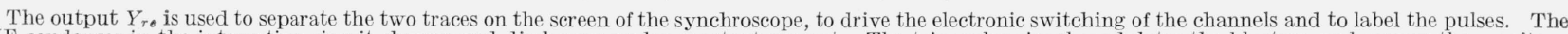

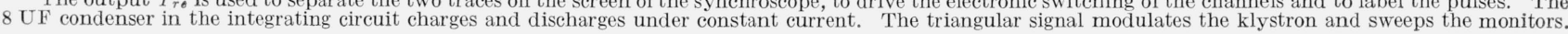




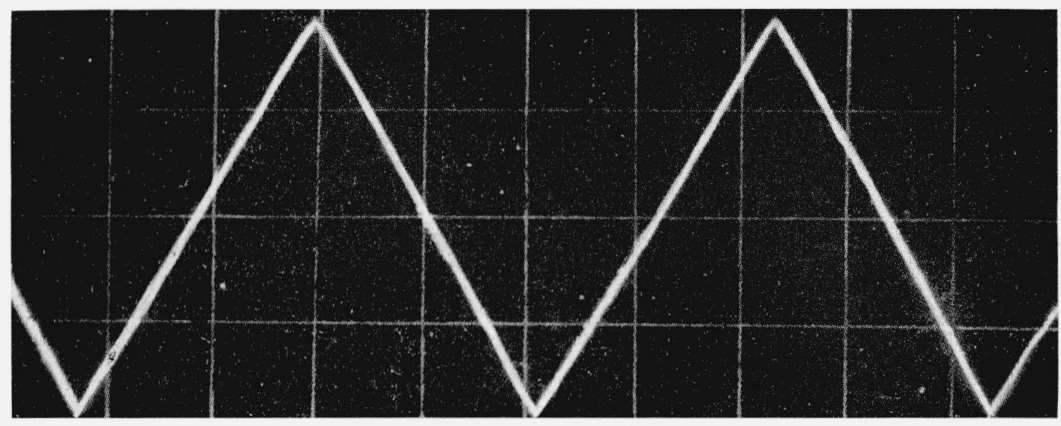

Figure 12. Shape of the triangular voltage produced by the modulator.

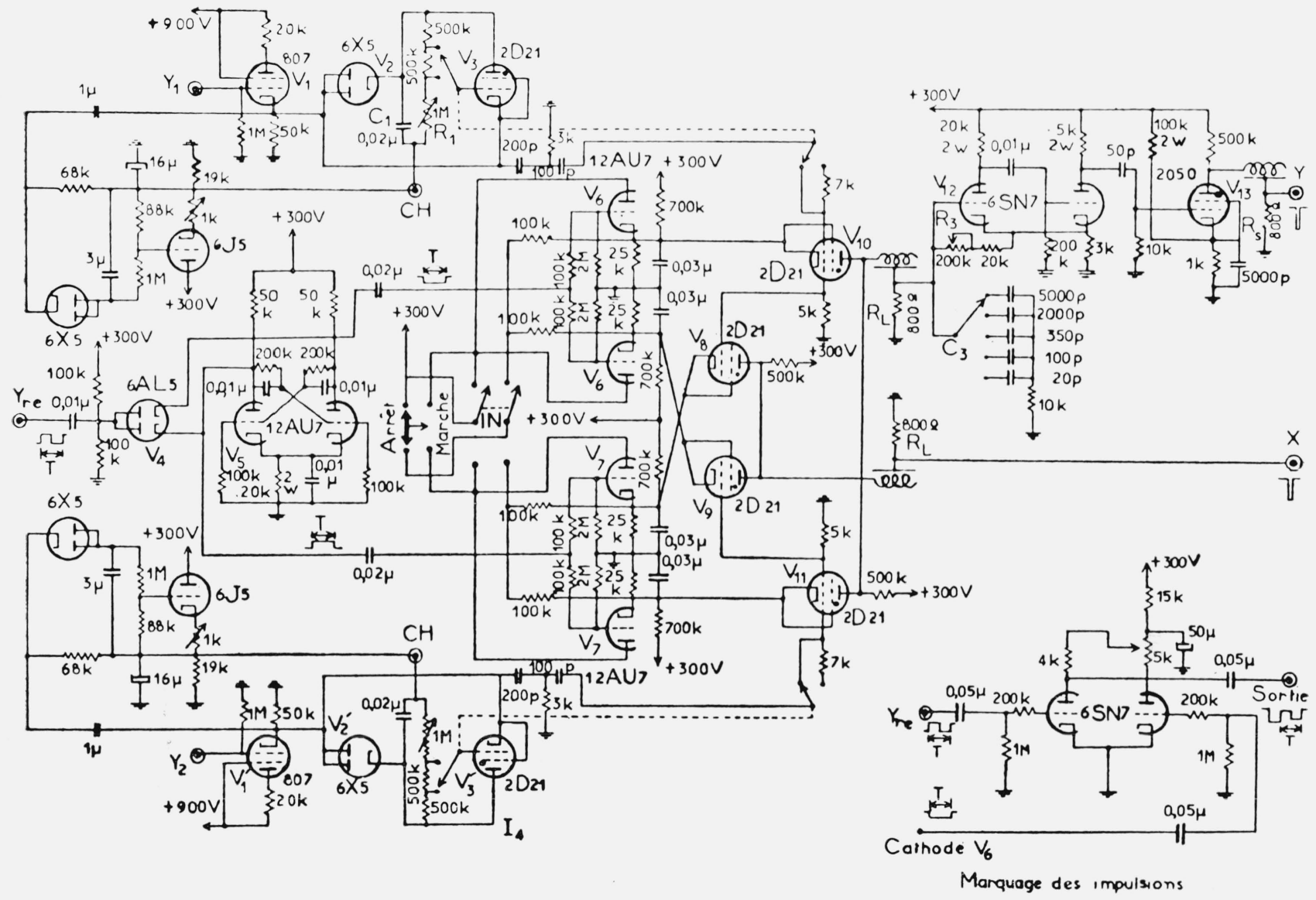

Figure 13. Diagram of the pulse generators circuits.

during one determined period, two pulses originating at one of the discriminators and during the following period two other pulses coming from the other discriminator. This insures the commutation between the two channels.

3. Impulse generators - The pulses produced on one of the resistors $R_{L}$ can be sent directly to the synchroscope. But those pulses which appear on the other resistor $R_{L}$ must be retarded by an amount of time $\tau ; \tau$ should be adjustable. These pulses fire the monostable multivibrator $V_{12}$ which furnishes the square pulse of duration $\tau$ adjusted by the value of the time constant $R_{3} C_{3}$ (40 to $\left.2500 \mu \mathrm{sec}\right)$. This pulse is differentiated and the signal which corresponds to the back of the signal fires the thyratron $V_{13}$. One obtains on resistor $\mathrm{R}_{S}$ a pulse which is sent to the synchroscope and which is retarded by $\tau$ with respect to the discriminator's pulse.

A few other improvements which we have incorporated in the pulse circuits can be resumed by making the following three remarks:

A corrective device to eliminate the effect produced by the variations of the width of the response of the cavity on the height of discrimination $a$-To realize this the voltages of the discriminator itself (6 X $5-2$ D 21) are reported not to the ground but 


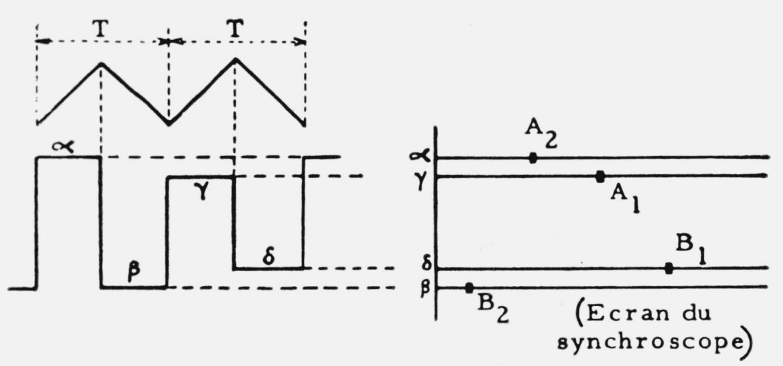

Figure 14. Operation of the circuits which insure the labeling of the pulses, operational scheme of the instrument.

to the cathode of an amplifier for direct current (6 $J 5)$. The input of this d-c amplifier receives a fraction of the voltage coming from the same response signal detected by a diode (6 X 5). Any variation of the continuous component of the response signal, which could effect the parameter $K$ of the instrument, is thus automatically compensated.

$A$ stop and go switch for the electronic commutator When this switch is in the OFF position, tubes $V_{9}$ and $V_{10}$ are saturated by a strong polarization (bias) while the other tubes $V_{8}$ and $V_{11}$ can operate continuously, the flip-flop signal being inoperative because the high voltage of tube $V_{7}$ has been interrupted. When the switch is in the OFF position, one can invert the roles of the two channels by means of the manual switch IN.

Pulses labeling - To distinguish the origin of each of the four pulses, a device split each trace on the screen of the synchroscope. The length of the splitting is adjustable from 0 to a certain value. The circuit of this device is shown in figure 13 and its operation on figure 14 (see also fig. 4). Each pulse will appear as a separate trace. The two pulses which appear on the two outside tracks come from the same channel while the two others come from the other channel. This can be used to determine, for instance, which of the two cavities has the higher Q-factor.

All the electronic circuitry has been built at the Laboratoire de Physique de l'Atmosphere.

\subsection{Adjustment and Calibration of the Instrument}

A. Adjustment-The tuning of the cavities to the frequency selected for the experiment must be made towards the center of the klystron mode to insure maximum linearity.

The amplifier gain is so adjusted that when the two impulse generators are triggered by the response signal of one of the amplifiers (which automatically discards all causes of fluctuation by background noise), the pulses on the screen of the synchroscope remain very stable. In this fashion one insures that the uncertainty on the firing of the thyratron does not introduce appreciable errors. The maximum voltage of the amplified response signal must not exceed a value of the order of 300 so as not to saturate the amplifiers. Once the gain has been adjusted to its optimum value and once the operation of the channel has been established, the positions of the pulses must be independent of the gain within rather large limits. The gain of the measuring channel is readjusted during the experiment so that the maximum voltages of the amplified responses are about the same for the two channels.

Finally, the condition $k_{1}=k_{2}=k_{3}$ is insured as indicated in paragraph 4.

B. Relative calibration - The index of refraction and the absorption are proportional to quantities which are actually measured during the experiment. Consequently, the constants of proportionality can be deduced from measurement made on gases whose index of refraction and the absorption as a function of pressure are sufficiently well known (argon for the index of refraction, ammonia for the absorption).

C. Absolute calibration-To accomplish this, one must determine directly the constants $\alpha$ and $k$ of the instrument.

To measure $v$, one impresses on the klystron the secondary frequency of modulation by applying to its reflector a voltage of radio frequency $f$ (see sec. 1.7 and fig. 11). Then one tunes the cavity on the first two lateral bands $\nu \pm f$ and one superimposes the pulses (two-pulse technique) on the screen of the synchroscope. By acting on the quartz oscillator one changes the radio frequency to $f+\Delta f$. The pulses are now separated and the distance of separation is that one would observe if the cavities were tuned, in the absence of modulation by radio frequency, at two frequencies differing by an amount $2(\Delta f)$. Equation (1.14) gives immediately the value of $v$. In practice one uses a frequency $f$ of a few $\mathrm{Mc} / \mathrm{s}$ and a difference $f$ of a few tens of $\mathrm{kc} / \mathrm{s}$.

To determine $k$, one sends the output signal of one of the amplifiers to the vertical plates of an oscilloscope and one sends the corresponding pulse, picked up at the output of the corresponding discriminator to the cathode of the tube of the same oscilloscope. A picture is taken of the oscilloscope screen. One obtains the curve shown on figure 15 and one tries to describe it by means of an equation $y=f(x)$. The shape of the response is thus determined (this is the same thing as the determination of the crystal detector law, square law for instance) and the height of discrimination is read on the photograph by pegging the position of the pulse on that curve. From these $k$ is deduced (sec. 1.3).

From a practical viewpoint it is preferable to measure the refraction index or the absorption by stages, noting the corresponding positions of the pulses and reading the pressure when the measuring cavity is progressively evacuated.

\subsection{Sensitivity, Stability, and Precision}

A. Sensitivity-The sensitivity of the instrument is limited by the fact that the pulses fluctuate on the screen of the synchroscope. This fluctuation is caused in the last analysis by the background noise from the output of the amplifier. The crystal detectors which feed the amplifier are mainly responsible for the noise. All other causes of fluctuation can be eliminated in practice. 


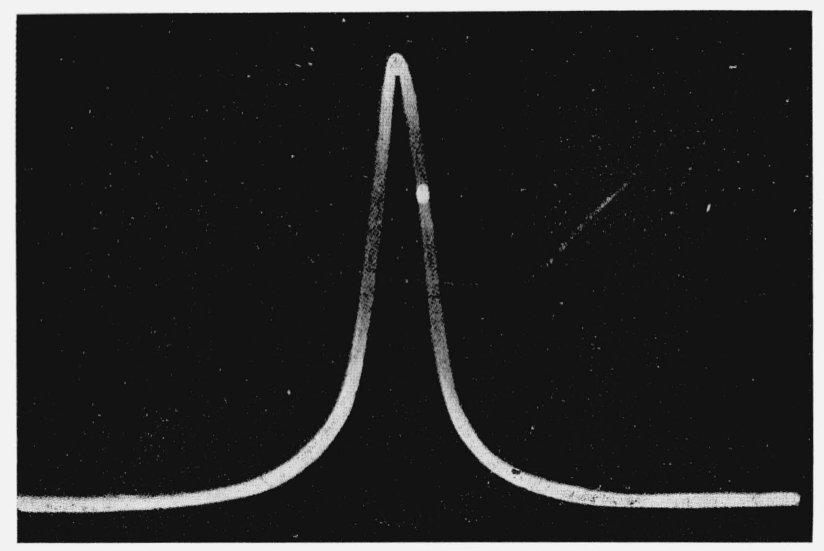

Figure 15. Determination of the constant $\mathrm{k}$ of the instrument.

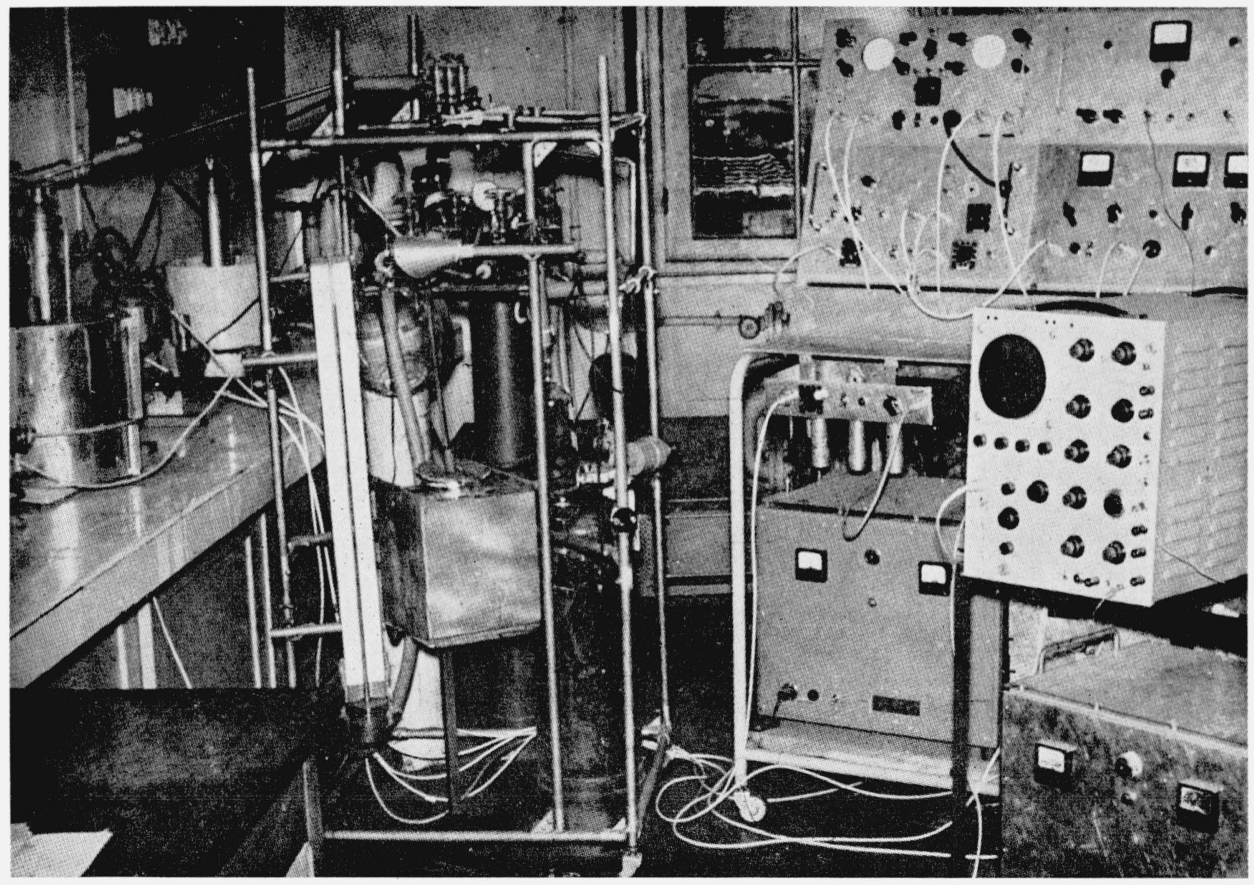

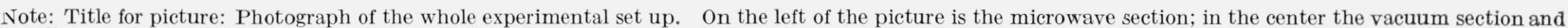

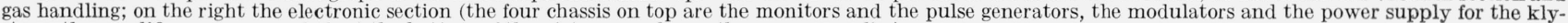
stron; the amplifiers are suspended; at the bottom of the picture are shown the power supplies).

The fluctuation of the output signal of the amplifiers brings about an uncertainty $\Delta \nu$ on the frequencies discriminated by the pulse generators. The instrument sensitivity is thus characterized by the ratio $\left(\frac{\Delta \nu}{\nu}\right)_{\min }$ which we are going to compute.

Let us suppose that the weakest observable effect is the one which produces a pulse separation of the same order of magnitude as the fluctuations. In this case the uncertainty $\Delta \nu$ in frequencies can be computed.

Let $N$ be the noise voltage at the output of one of the amplifiers, $G$ be the amplifiers' gain, and $V(P)$ be the tension at the terminals of the crystal detector. This tension depends upon the power $P$ applied to the crystal. We will have:

$$
N=G \frac{d V}{d P} \cdot \frac{d P}{d \nu} \cdot(\Delta \nu),
$$

$\Delta \nu$ is defined above and $d V / d P$ and $d P / d \nu$ are derivatives which one computes at frequencies $\nu^{+}$ and $\nu^{-}$for which $V / V_{\max }$ admits to a determined value $a_{1}$ or $a_{2}$. From (1.4), (1.5), (1.6), (1.7), and (1.32) one deduces that $\left(\frac{\Delta \nu}{\nu}\right)$ is minimum if the condition:

$$
a=\left(\frac{2+m}{2(m+1)}\right)^{m}
$$


is realized. $1 / m$ is the exponent fixing the detection law of the crystal (1.5). For a quadratic detection ( $m=1$ ) one finds $a=3 / 4$, while for a linear detection ( $m=2$ ) one finds $a=4 / 9$ or about $1 / 2$.

If the power transmitted by the cavity is sufficiently weak $\left(P_{\max }<10 \mu \mathrm{w}\right)$, one has [Gordy, Smith, and Trambarulo, 1953, p. 68]:

$$
N=G \sqrt{k T\left(R_{A}+R\right) B},
$$

where $R_{A}$ is the video resistance of the crystal, $R$ the equivalent resistance of the amplifier and $B$ its band pass ( $k$ Boltzmann constant and $T$ the absolute temperature). From (1.4), (1.32), and (1.34) one has

$$
\left(\frac{3 \sqrt{3}}{4}\right) S Q P_{\max }\left(\frac{\Delta \nu}{\nu}\right)_{\min }=\sqrt{k T\left(R_{A}+R\right) B}
$$

where one assumes $a=3 / 4$ (quadratic detection). If one introduces the "merit factor" $M$ of the video receiver, made up of the crystal and the amplifier

$$
M=\sqrt{\frac{S}{R+R_{A}}},
$$

one will have

$$
\left(\frac{\Delta \nu}{\nu}\right)_{\min }=\frac{4}{3 \sqrt{3}} \frac{\sqrt{k T B}}{M} \frac{1}{Q P_{\max }} .
$$

Taking into account (1.1) and (1.30) one obtains:

$$
\left.\begin{array}{l}
(\Delta n)_{\min }=\left(\frac{\Delta \nu}{\nu_{c}}\right)_{\min } \\
\alpha_{\min }=\frac{2 \pi \nu_{c}}{k c}\left(\frac{\Delta \nu}{\nu_{c}}\right)_{\min }
\end{array}\right\}
$$

$\nu_{c}$ being the resonance frequency of the cavities. Equation (1.39) shows that the smallest detectable absorption depends upon the frequency, because the physical phenomenon used to detect absorption depends on the frequency. In all cases, the sensitivity will be greater for large values of $Q$, those two quantities being directly proportional.

In the frequency band $10 \mathrm{Gc} / \mathrm{s}$ (band $\mathrm{X}$ ) one obtains by using selected crystals, values of $M \approx 100$. By taking $Q \approx 30,000$ and $G \approx 10 \mathrm{kc} / \mathrm{s}$ and $T \approx 300^{\circ} \mathrm{K}$, (1.37) gives:

$$
\left(\frac{\Delta \nu}{\nu}\right)_{\min } \approx 2 \cdot 10^{-9}
$$

In practice the power incident on the crystal can become larger, and if one adjusts it experimentally to its optimum value, the sensitivity can be improved.

To adjust the instrument for high sensitivity, one must appropriately reduce the velocity of modulation of the klystron. One will thus reduce the amplitude (and eventually the frequency itself) of the voltage of triangular modulation. For our instrument, the modulation frequency is fixed at $50 \mathrm{c} / \mathrm{s}$ (another modulator for demultiplication of frequency is planned) and the peak-to-peak voltage of the triangular signal may vary from a few volts (maximum sensitivity) to about $40 \mathrm{v}$ (minimum sensitivity). One works efficiently around $20 \mathrm{v}$ (average sensitivity, but often sufficient).

Under the most favorable conditions, one can detect a variation of $10 \mathrm{c} / \mathrm{s}$ on a background of $10 \mathrm{Gc} / \mathrm{s}\left(\Delta \nu / \nu \approx 10^{-9}\right)$. The maximum sensitivity is thus from $10^{-8}$ to $10^{-9}$ for the index of refraction and of $10^{-6}$ neper $/ \mathrm{m}$ for the absorption ( $\mathrm{X}$ band).

B. Stability-If one takes elementary precautions the instrument's stability is very good. The fluctuation of pulses on the synchroscope screen will remain less than one $\mu$ sec for long periods of time (modulation speed $v \approx 30 \mathrm{Mc} / \mathrm{s}^{2}$ ). Calibration can be reproduced within 1 or 2 percent from one day to the next.

The necessary precaution needed to assure maximum stability can be listed as follows: avoid all high frequency energy leaks, take good care of the movable mechanical components of the microwave section, select your crystals carefully, avoid all mechanical vibrations, pay careful attention to all feed-line problems (good stabilization, independent power for each chassis, and for each channel so as to eliminate all interaction, partial use of a battery to heat the tubes' filaments), control the cavities thermally.

C. Precision-Since most sources of errors which have plagued other types of instruments designed for this same usage, have been eliminated in our apparatus, the finer precision is limited by the determination of the pressure of the gas under study and inaccuracies inherent to every measurement in vacuum (adsorption effect, preparation of mixtures, behavior of each gas). The pressure is read on a mercury barometer, with the help of a micrometric device and one reaches precision of the order of 0.2 percent in the neighborhood of $100 \mathrm{~mm} \mathrm{Hg}$.

When making absolute measurement, one can determine the speed of modulation $v$ with an accuracy of a few parts in a thousand, while the error on the parameter $k$ is of the order of 1 percent (see sec. 1.9).

The final precision depends largely on the type of measurements made. To gain a feel for the precision expected, one should read the chapter devoted to results and their discussions. We will note, however, that the total precision for absorption measurement is of the order of 0.5 to 2 percent, while for the index of refraction measurements where the parameter $k$ is not needed, the precision under favorable conditions is of the order of $a_{5}$ few parts in a thousand.

The instrument which we have just described could be improved and could be transformed in many ways. For instance, we are studying now how the resulting measurements could be automatically and continuously registered on a paper chart. Such a study is being made at the Laboratoire de Physique de l'Atmosphere. 


\section{Theory of the Index of Refraction of Absorption and Dispersion}

This chapter is not intended to be a complete resume of the many theories concerning the dielectric constant, the index of refraction, the absorption and dispersion of radiation by matter. However, the literature on these fundamental questions of physies is so vast that it is often inconvenient to consult it and we want to present here, as clearly and coherently as possible, the essential part of those theories which are of interest to us. This is necessary because most authors consider these subjects from a specific but often different viewpoint (frequency band, state of the matter, electric viewpoint, or spectroscopic viewpoint) and they introduce convenient notations and systems of units. Our field is that of hyperfrequency (from a few $\mathrm{Gc} / \mathrm{s}$ to a few tens of $\mathrm{Gc} / \mathrm{s}$ ), matter is assumed in the gaseous state, the spectroscopic viewpoint is often the dominant one and the M.O.K.S. (or Giorgi) system of units is used all along in this work except when otherwise specified.

\subsection{Molecular Interpretation of the Dielectric Properties of a Gas}

Debye [1912, 1925, 1929, and 1954]; Fröhlich [1949, 1956, and 1948]; Smyth [1955]; and Van Vleck [1932].

A. The Clausius-Mosotti relation - One shows that for an isotropic, homogeneous and nonpolar gas one has

$$
\frac{\epsilon_{r}-1}{\epsilon_{r}+2}=\frac{\alpha N}{3 \epsilon_{0}}
$$

$\boldsymbol{\epsilon}_{r}$ is the dielectric constant of the gas with respect to vacuum (for the time being it is a real number), $\alpha$ its induced molecular polarization (induced electric moment per molecule and per unity of field), $N$ the number of molecules per unit volume (per $\mathrm{m}^{3}$ ) and $\epsilon_{0}=8.8545 \times 10^{-2} \mathrm{farad} / \mathrm{m}$ (the permittivity of vacuum in the rationalized MKS system of units).

This relation assumes that the molecules interact weakly with each other and that the applied electric field is not too strong so that the elastic displacement of the charge centers of the molecule are proportional to the field.

One can then write the same relation as:

$$
R_{M}=\frac{\epsilon_{r}-1}{\epsilon_{r}+2} \frac{M}{\rho}=\frac{\epsilon_{r}-1}{\epsilon_{r}+2} \frac{R T}{p_{i}}=\frac{\alpha N_{A}}{3 \epsilon_{0}},
$$

$R_{M}$ is the molecular refractivity of the gas, $M / \rho$ its specific molecular volume $\left(22.414 \mathrm{~m}^{3}\right.$ at $0^{\circ} \mathrm{C}$ and 760 $\mathrm{mm} \mathrm{Hg}$ ), $p_{i}$ the gas pressure in newtons per $\mathrm{m}^{2}$ (the gas is assumed ideal), $T$ its absolute temperature in ${ }^{\circ} \mathrm{K}$ and $R=8.314 \times 10^{3}$ joule $/{ }^{\circ} \mathrm{K} M\left(\mathrm{~kg}^{2}\right), N_{A}=6.023$ $\times 10^{26}$ molecules per $M \mathrm{~kg}$ (Avogadro's number).

Equation (2.2) expresses the law of ClausiusMosotti. If one supposes that in the case of gases $\epsilon_{r}$ is very close to unity and if one uses the relation: $N=\frac{N_{A} \rho}{M}=\frac{N_{A}}{R} \cdot \frac{p_{i}}{T}$, or: $N=0.96525 \cdot 10^{25} \cdot \frac{p_{i}}{T},\left(m^{-3}\right)$, where $p_{i}$ is measured in $\mathrm{mm} \mathrm{Hg}$, one finally obtains :

$$
(n-1) \cdot 10^{6}=54.52 \cdot 10^{40} \alpha \frac{p_{i}}{T},
$$

where the index of refraction $N$ is introduced to the relation:

$$
\left.\epsilon_{r}-1=n^{2}-1 \approx 2(n-1) \text { (refer to sec. } 2.2 \mathrm{~A}\right) .
$$

This relation requires that the index $(n-1) \times 10^{6}$ be proportional to the gas pressure and inversely proportional to the absolute temperature insofar as the induced molecular polarization $\alpha$ is a constant which depends only on the molecular structure of the gas under study. We must not lose sight of the fact that this result has been obtained by making a certain number of hypotheses and assumptions.

The law of Clausius-Mosotti has received within the limits of its application, numerous experimental verifications; these include our own measurement. The theory of the dielectric constant of nonpolar gas has been improved during these last few years [Hill, 1958; Jansen and Mazur, 1955; Jansen and Solem, 1956; Mandel, 1958; Mandel and Mazur, 1958; Mazur and Mandel, 1956a and 1956b].

B. The Debye-Langevin relation - The molecular polarization of polar gas is made up of two terms: one is the result of induction, the other resulting from the orientation of the permanent moments $\mu$ of the molecules. The total molecular polarization is (if $\mu E<k T$ ):

$$
\alpha_{t}=\alpha+\left(\mu^{2} / 3 k T\right),
$$

where $K=1.3804 \times 10^{-23}$ joule $/{ }^{\circ} K$ is the Boltzmann constant

If one substitutes $\alpha$ for $\alpha$ in (2.2) one obtains the Debye-Langevin relation. The same substitution could be made into (2.4)

The computation of the total polarization has been taken up again using quantum mechanical methods. Van Vleck [1932 (ch. VII)] obtained a relation almost identical with (2.5) with this difference that the term $\mu^{2} / 3 k T$ is multiplied by a correction factor $(1-f(T))$. This factor is usually very close to unity and we will not have to take it into account when we interpret our results.

The law of Debye-Langevin predicts, for polar substances, a decrease of the dielectric constant with temperature. This decrease will be faster than $1 / T$. Many experiments verify this prediction and in a general way this law does express, within the limits of its validity, the dielectric constant of gases for ordinary pressures. It is approximately valid for the solutions of polar substances in nonpolar solvents. But it is not valid any more for pure polar liquids. For these it predicts a saturation effect which is too rapid. It is a kind of "ferroelectric" effect rarely observed in certain solids. Several corrected formulas have been suggested to remedy this fault but we will not discuss them here.

C. Nonresonant absorption of the Debye type- So far we have considered only the static case. The 
applied field frequency is assumed null or low enough so that the dielectric is at all times in equilibrium with the field.

If the field frequency increases, it gets out of phase with the effect (induction orientation) on the dielectric. Equilibrium will be reached after the applied field has been removed in accordance with a law of the type $\exp (-t / \tau)$ where $\tau$ is the relaxation time. It is independent of $t$ but does depend upon the temperature.

Debye [1929, 1912, 1925, and 1954] has derived most of the classical relations which define quantities such as power losses caused by relaxation phenomenon ( $\epsilon_{r}^{\prime \prime}$ is the loss factor), the dielectric constant which has now become a complex number $\boldsymbol{\epsilon}_{r}=\epsilon_{r}^{\prime}-$ $i \epsilon_{r}^{\prime \prime}$, the field frequency, the molecule dimension and the "viscosity" of the dielectric medium. From this work we quote the following expression for the complex dielectric constant [Debye, 1929, 1912, 1925, and 1954; Fröhlich, 1949, 1956, and 1948]:

$$
\left.\begin{array}{c}
\epsilon_{r}^{\prime}=\epsilon_{r \infty}+\frac{\epsilon_{r 0}-\epsilon_{r \infty}}{1+(\omega \tau)^{2}} \\
\epsilon_{r}^{\prime \prime}=\frac{\left(\epsilon_{r 0}-\epsilon_{r \infty}\right) \omega \tau}{1+(\omega \tau)^{2}}
\end{array}\right\}
$$

$\boldsymbol{\epsilon}_{r 0}$ and $\boldsymbol{\epsilon}_{r \infty}$ are the dielectric constants (real numbers) measured for frequencies $\omega \rightarrow 0$ or $\omega \rightarrow \infty$. The loss factor $\epsilon_{\nu}^{\prime \prime}$ corresponds to a power absorption of the nonresonant type, it becomes maximum for a critical frequency $\omega_{m}$ such that $\omega_{m} \tau=1$ [Foley, 1942].

\subsection{Index of Refraction, Absorption, and Normal Dispersion}

Stratton [1941]; Bruhat [1954]; and Boudouris [1957].

A. Definitions - The index of refraction of a medium is defined by

$$
n=\sqrt{\epsilon_{r} \mu_{r}}=n^{\prime}-i n^{\prime \prime} ;
$$

where the dielectric constant and the magnetic permeability of the medium are usually complex numbers. Consequently, the index of refraction will also be complex.

The real part $n^{\prime}$ of the index, often called index of refraction and designated by $n$, determines the phase velocity of the wave $\left(v=c / n^{\prime}, \quad c=2.9979 \times 10^{8}\right.$ msec). The imaginary part $n^{\prime \prime}$ (coefficient of extinction) determines the absorption of the transmitted power and one has:

$$
\alpha=\frac{2 \omega}{c} n^{\prime \prime}=\frac{4 \pi \nu}{c} n^{\prime \prime},(\text { neper } / \mathrm{m}),
$$

where $\alpha$ is the coefficient of absorption for the medium and $\nu$ the frequency of the applied field.

We should notice that the concept of index of refraction of a medium is intimately tied to phenomenas of electromagnetic wave propagation, while the constant $\epsilon_{r}$ and $\mu_{r}$ are "static" in origin and must be deduced from separate electrical and magnetic measurements. A knowledge of the index of refraction does not in general imply a knowledge of $\boldsymbol{\epsilon}_{r}$ and $\mu_{r}$. It is only if one admits that $\mu_{r}=1$, as we shall assume in the following treatment, that one has:

$n=\sqrt{\epsilon_{r}}=n^{\prime}-i n^{\prime \prime}, \epsilon_{r}=\epsilon_{r}^{\prime}-i \epsilon_{r}^{\prime \prime}=n^{2}, \quad\left(\mu_{r}=1\right)$.

The result of this is (if, as is the case for gases, $\left.n^{\prime \prime}<<n^{\prime} \approx 1\right)$ :

$$
\begin{array}{r}
\epsilon_{r}^{\prime}=n^{\prime 2}-n^{\prime \prime 2} \approx n^{\prime 2}, \epsilon_{r}^{\prime \prime}=2 n^{\prime} n^{\prime \prime} \approx 2 n^{\prime \prime}=\frac{c}{2 \pi \nu} \alpha, \\
\left(\mu_{r}=1\right) .
\end{array}
$$

B. Normal dispersion-The fundamental problem of the interaction between matter and radiation can be treated from the viewpoint of classical physics or from the view point of quantum physics. Taking the classical physics viewpoint one must select a microscopic model which will represent as well as possible the matter's structure. Since our aim is to discuss the phenomenon of dispersion (where the index of refraction is a function of frequency), we will take a very simple model. The molecule is made up of "charge centers" of charge $q$ and mass $m$, these centers will be displaced by the influence of an effective field $E_{\text {eff. }}$. The displacement $s$ is perfectly elastic and devoid of any friction. The equation of motion is

$$
m\left(\partial^{2} s / \partial t^{2}\right)+k s=q E_{\mathrm{eff}},
$$

where $k$ is a measure of the elastic forces (the vectorial sign will be omitted above the quantities $E$, $s$. . . because they are all parallel to each other). The frequency of the characteristic oscillations is

$$
\omega_{0}=2 \pi \nu_{0}=\sqrt{k / m}
$$

and the equation of motion becomes:

$$
\left(\partial^{2} s_{/}^{\prime} \partial t^{2}\right)+\omega_{0}^{2} s=(q / m) E_{\text {eff }} .
$$

Under the influence of the field $E_{\text {eff }}=E_{\text {eff }}^{0} \times$ $\exp (i \omega t)$, the charge center will oscillate according to the law $s=s_{0} \exp (i \omega t)$. The equation of motion admits the particular solution:

$$
\frac{s}{E_{\mathrm{eff}}}=\frac{q}{(2 \pi)^{2} m} \frac{1}{\nu_{0}^{2}-\nu^{2}} .
$$

The polarization per unit of volume can be expressed by the relation:

$$
p_{v}=N q s=\epsilon_{0}\left(\epsilon_{r}-1\right)\left(E_{\mathrm{eff}}-\frac{p_{v}}{3 \epsilon_{0}}\right) .
$$

It results from the above relations that:

$$
\epsilon_{r}=1+X \frac{\nu_{0}^{2}}{\nu_{0}^{2}\left(1-\frac{1}{3} X\right)-\nu^{2}} \approx 1+X \frac{\nu_{0}^{2}}{\nu_{0}^{2}-\nu^{2}}
$$




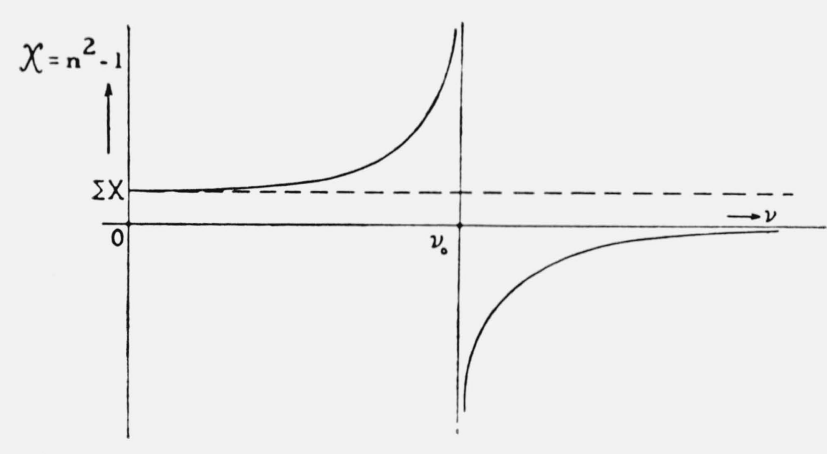

Figure 16. Normal dispersion curve.

with

$$
X=\frac{q^{2} N}{(2 \pi)^{2} \nu_{0}^{2} \epsilon_{0} m}, \text { (without dimensions), }
$$

the second equation (2.16) gives a good approximation for the case of gaseous media.

If the molecules making up the medium present an assembly of charges of the type $(q, m)$, all of them subjected to the action of a field, we will have (Sellmeier's relation):

$$
\epsilon_{r}-1=n^{2}-1=\sum X \frac{\nu_{0}^{2}}{\nu_{0}^{2}-\nu^{2}}=\sum X+\sum X \frac{\nu^{2}}{\nu_{0}^{2}-\nu^{2}} .
$$

Here the dielectric constant and the index of refraction are real numbers since all causes of absorption have been discarded. The quantity $\Sigma X$ is equal to $\epsilon_{\tau 0}-1, \epsilon_{\tau 0}$ being the static dielectric constant measured for $\nu \rightarrow 0$.

Equation (2.18) and figure 16 show, for a typical case, how the index of refraction varies with frequency (normal dispersion). Here we notice the anomaly according to which the index of refraction would become infinite for the resonance frequency $\nu=\nu_{0}$. This is caused by the fact that we neglected all absorption effects. This, as we shall see later, will determine the real behavior of the index of refraction in the neighborhood of the resonance frequency.

\subsection{Resonant Absorption and Âbnormal Dispersion}

\section{(Theory of Van Vleck-Weisskopf)}

Bruin [1956]; Fröhlich [1949, 1956, and 1948]; Gordy, Smith and Trambarulo [1953]; Townes and Schalow [1955]; and Van Vleck and Weisskopf [1945].

The original Lorentz theory of the shape of spectrolines for the visible [Lorentz, 1906] has been properly modified so as to be compatible with the Debye theory [1929 (sec. 2.1C)] and to be valid in the hertzian domain. This work has been done by Van Vleck and Weisskopf [1945]. We are going to discuss the parts of this modified theory which are essential for the interpretation of the absorption and dispersion produced by molecular resonance. The other theories concerning the shock broadening of spectral lines will be reviewed.

A. Theory of Van Vleck-Weisskopf (VV-W) The process of radiation absorption by matter is influenced by several factors. In the hertzian domain the most important of these factors is the effect produced by collisions between molecules. When two molecules (eventually several) come near enough to each other to be subject to intermolecular forces we say that collisions have occurred. This does not necessarily imply a direct collision between molecules which, in the kinetic theory of gas, had been represented by little spheres. The following hypotheses are made:

1. The gas pressure is low enough so that molecules collide only two at a time. The possibility of triple or high order collisions is discarded.

2. The collisions have a short duration:

$$
\tau_{c} \ll \tau,
$$

$\tau_{c}$ is the mean duration of a collision. It depends upon the type of molecular interaction. $\tau$ is the average time between collisions.

3. The collisions are strong. Consequently, the initial condition characterizing the motion of a molecule immediately after a collision bears no relation to those existing immediately before the collision. We say that the process of molecular interaction has no memory.

4. The initial conditions are, however, influenced by the intensity of the radiation field at the time of the shock. For this to be true we must have

$$
\tau_{c}<1 / \omega
$$

This tells us that the results of this theory will be valid only for frequencies which are sufficiently low.

5. The initial conditions characterizing the motion just after a collision are determined under the assumption that the positions and velocities of the charge centers of the molecules reach a Boltzmann distribution (thermal equilibrium is supposedly maintained).

The equation of motion for the charge centers subjected to the action of an external field will be

$$
\left(\partial^{2} s / \partial t^{2}\right)+\omega_{0}^{2} s=(q / m) E,
$$

during the interval of time $\tau$ which separates two successive collisions and if the five assumptions hold. This equation is identical to (2.13) where $\omega_{0}=2 \pi \nu_{0}$ is the characteristic frequency of the oscillating particle. We note that no friction term appears in the equation. The results, however, will produce absorption lines which are collision-broadened by shock. This happens because the transient terms which had been neglected in the solution of the particular (2.14) 
have been kept:

$$
s(t)=\frac{q E_{0} \exp (i \omega t)}{(2 \pi)^{2} m\left(\nu_{0}^{2}-\nu^{2}\right)}+C_{1} \exp \left(i \omega_{0} t\right)+C_{2} \exp \left(-i \omega_{0} t\right) .
$$

This general solution supposes that the particle under consideration oscillates with a constant amplitude between two successive collisions. However, the phase of the oscillation can be modified by collisions and a Fourier analysis would indicate the appearance of new frequencies of absorption around the resonance frequency. It is in this fashion that one talks of the shock or collision-broadening of absorption lines.

The constants $C_{1}$ and $C_{2}$ are determined with the help of the initial conditions: position $s\left(t_{0}\right)$ and velocity $v\left(t_{0}\right)=(d s / d t)_{t=t_{0}}$ of the oscillating particle at time $t_{0}$ right after a collision. Under the assumption of a Boltzmann distribution one obtains for the average value of $s\left(t_{0}\right)$ :

$$
\left\langle s\left(t_{0}\right)\right\rangle=\int s \cdot \exp (-H / k T) d s d v / \int \exp (-H / k T) d s d v,
$$

where

$$
H=(1 / 2) m v^{2}+(1 / 2) m\left(\omega_{0} s\right)^{2}-q s E_{0} \cos \omega t_{0},
$$

hence:

$$
\left\langle s\left(t_{0}\right)\right\rangle=\left(q E_{0} / m \omega_{0}^{2}\right) \exp \left(i \omega t_{0}\right) \text {, and }\left\langle v\left(t_{0}\right)\right\rangle=0 .
$$

Taking into account these initial conditions, one obtains from $(2.22)$

$$
\begin{array}{r}
s(t)=\frac{q E_{0} \exp (i \omega t)}{m \omega_{0}^{2}}\left(\frac{\nu_{0}^{2}}{\nu_{0}^{2}-\nu^{2}}-\frac{1}{2} \frac{\nu}{\nu_{0}-\nu} \exp \left\{-i\left(\omega-\omega_{0}\right) t^{\prime}\right.\right. \\
\left.+\frac{1}{2} \frac{\nu}{\nu_{0}+\nu} \exp \left\{-i\left(\omega+\omega_{0}\right) t^{\prime}\right\}\right), \quad(2.26)
\end{array}
$$

where $t^{\prime}=t-t_{0}$ is the time measured since the last collision. To arrive at the expression for the mean displacement $\langle s(t)\rangle$ we must compute the average value of (2.26) with respect to $t^{\prime}$ considered as the time at which the next collision occurs.

According to the kinetic theory of gas [Kennard, 1938] the probability $w$ for a molecule to collide after a time $t^{\prime}$ following its last collision is $w=(1 / \tau)$ $\exp \left(-t^{\prime} / \tau\right)$. We then have $\langle s(t)\rangle=\int_{0}^{\infty} s \cdot w \cdot d t^{\prime}\left(t^{\prime}\right.$ variable, $t$ is fixed) which leads after integration to:

$$
\langle s(t)\rangle=\frac{q E_{0} \exp (i \omega t)}{m \omega_{0}^{2}} \frac{(1+i \omega \tau)+\left(\omega_{0} \tau\right)^{2}}{(1+i \omega \tau)^{2}+\left(\omega_{0} \tau\right)^{2}} .
$$

The knowledge of the mean displacement gives us (see $(2.15)$ :

$$
\epsilon_{r}=1+X \frac{(1+i \omega \tau)+\left(\omega_{0} \tau\right)^{2}}{(1+i \omega \tau)^{2}+\left(\omega_{0} \tau\right)^{2}}, \quad\left(X=\frac{q^{2} N}{\omega_{0}^{2} m \epsilon_{0}}\right) .
$$

This classical result can be transformed [Van Vleck and Weisskopf, 1945] with the help of the correspondence principle, so as to become valid in the domain of quantum mechanies [Heitler, 1954; Pauling and Wilson, 1935; Ruark and Urey, 1930; Schiff, 1949].

The classical resonance frequency $\nu_{0}$ is first considered to be equal to the quantic resonance frequency $\nu_{i j}$ expressed by the Bohr relation:

$$
\nu_{0}=\nu_{i j}=\left(E_{j}-E_{i}\right) / h,
$$

where $h=6.6252 \times 10^{-34}$ joule $\cdot$ sec is the Planck constant, $E_{i}<E_{j}$ the energies of levels $i$ and $j$ of the molecule between which the absorbing transition is taking place. The rotational spectrum of a symmetric molecule, for instance, is made up of lines whose resonance frequencies are

$$
\nu_{i j}=2 B(J+1), \quad(B=3302 \mathrm{Mc} / \mathrm{s} \text { for chloroform })
$$

(sec. 3.2).

$B$ is the rotational constant of the molecule and $J=0,1,2, \ldots$ the quantic number of the lower levels of each transition $J \rightarrow J+1$ [Townes and Schalow, 1955].

To the quantity $q^{2} / \omega_{0}^{2} m$ which appears in the classical expression for $X(2.28)$ we substitute the quantum quantity $2\left|\mu_{i j}\right|^{2} / 3 k T$, since thermal equilibrium is assumed. This substitution brings in the matrix element of the dipole moment $\left|\mu_{i j}\right|$ which is called the dipole moment for the transition $i \rightarrow j$. If one admits that the classical dipole moment is directed along a principal axis of inertia (a) of the molecule, we will have along another fixed direction $x$ in space:

$$
\left.\begin{array}{l}
\left|\mu_{i j}\right|_{x}=\left(i\left|\mu_{x}\right| j\right)=\int \psi_{i} \mu \cos (\alpha, x) \psi_{j}^{*} d \tau, \\
\left(\left|\mu_{i j}\right|^{2}=\left|\mu_{i j}\right|_{x}^{2}+\left|\mu_{i j}\right|_{y}^{2}+\left|\mu_{i j}\right|_{z}^{2}\right),
\end{array}\right\}
$$

$\psi_{i}$ and $\psi_{j}$ being the wave function corresponding to states $i$ and $j$. The factor determines the selection rules for the transitions: a transition is "allowed" if one at least of the components of the matrix $\left|\mu_{i j}\right|$ along the axis $x, y$, and $z$ is different from zero [Gordy, Smith, and Trambarulo, 1953; Townes and Schalow, 1955].

Instead of the total number of molecules, $N$, per unit volume, we will introduce into (2.28) the quantity $N f_{i j}, f_{i j}$ being that fraction of the total number of molecules which are in the lowest state $i$. Writing these relations by means of an equality we have

$$
X=\frac{q^{2} N}{\omega_{0}^{2} m \epsilon_{0}}=\frac{2 N f_{i j}\left|\mu_{i j}\right|^{2}}{3 \epsilon_{0} \cdot k T} .
$$

This substitution allows us to pass from the classical formulae (2.28) to the corresponding quantic relations. At the same time the quantum mechanical expressions acquire a more general meaning; they become valid for any dipole transition (between vibrational states, rotation states, etc. . . .) and lend themselves to the determination of the absolute value 
of the absorption. Thus the problem reduced itself to the evaluation of the quantities $\nu_{i j}, \mu_{i j}$, and $f_{i j}$. Although we shall give some detail for the particular eases considered in the chapter titled "Results and Discussion" (3.2), one would do well to consult references on hertzian spectroscopy if one wishes to acquire more complete information [Gordy, Smith, and Trambarulo, 1953; Ingram, 1955; Townes and Schalow, 1955].

The final expression for the absorption and the index of refraction are obtained in the following manner: one introduces into $(2.28)$ the substitutions indicated by (2.29) and (2.32), one then separates the real part from the imaginary part $\epsilon_{r}$, one takes (2.10) into account and one sums over all possible transitions $i \rightarrow j$. The following formulas, due to Van Vleck-Weisskopf (VV-W), are thus obtained (rationalized MKS units):

$$
\begin{aligned}
& \alpha=A_{p} \nu^{2} \sum_{i j}\left(f_{i j}\left|\mu_{i j}\right|^{2} \cdot\left(F_{i j} / \nu_{i j}\right)\right),(\text { neper } / \mathrm{m}), \\
& \text { and } \\
& A_{p}=2 \pi N / 3 c \epsilon_{0} k T \\
& F_{i j}=\nu_{i j}(\Delta \nu)_{i j}\left(\frac{1}{\left(\nu_{i j}-\nu\right)^{2}+(\Delta \nu)_{i j}^{2}}\right. \\
& \left.+\frac{1}{\left(\nu_{i j}+\nu\right)^{2}+(\Delta \nu)_{i j}^{2}}\right)
\end{aligned}
$$

with

$$
n-1=B_{p} \sum_{i j}\left(f_{i j}\left|\mu_{i j}\right|^{2} G_{i j}\right)+\bar{n},
$$

and

$$
B_{p}=(c / 2 \pi) A_{p}=N / 3 \epsilon_{0} k T
$$

$G_{i j}=\frac{\nu_{i j}^{2}}{\nu_{i j}^{2}-\nu^{2}}\left[1-\frac{\nu(\Delta \nu)_{i}^{2}}{2 \nu_{i j}^{2}}\left(\frac{\nu_{i j}+\nu}{\left(\nu_{i j}-\nu\right)^{2}+(\Delta \nu)_{i j}^{2}}\right.\right.$

$$
\begin{aligned}
\left.\left.-\frac{\nu_{i j}-\nu}{\left(\nu_{i j}+\nu\right)^{2}+(\Delta \nu)_{i j}^{2}}\right)\right] \equiv & \frac{1}{2}\left[\frac{\nu_{i j}\left(\nu_{i j}+\nu\right)+(\Delta \nu)_{i j}^{2}}{\left(\nu_{i j}+\nu\right)^{2}+(\Delta \nu)_{i j}^{2}}\right. \\
& \left.+\frac{\nu_{i j}\left(\nu_{i j}-\nu\right)+(\Delta \nu)_{i j}^{2}}{\left(\nu_{i j}+\nu\right)^{2}+(\Delta \nu)_{i j}^{2}}\right] .
\end{aligned}
$$

In these relations we have taken:

$$
\Delta \nu=1 / 2 \pi \tau,
$$

$\tau$ is the time required by a molecule to cover a distance equal to the mean free path and $\Delta \nu$ is the line width parameter which is assumed to be proportional to the gas pressure although this is not always the case. $F_{i j}$ and $G_{i j}$ are dimensionless shape factors. For $F_{i j}$ we prefer the definition given by (2.35) which renders this factor a dimensionless quantity, slightly different definitions appear in the literature [Bleaney and Penrose, 1947; Van Vleck and Weisskopf, 1945].

Let us note that, in accordance with the principle of spectroscopic stability, the integrated intensity of line, defined by $\int_{0}^{\infty}\left(\alpha / \nu^{2}\right) d \nu$, must be independent of
$\Delta \nu$ and of the process responsible for the line broadening [Townes and Schalow, 1955]. The eq (2.33) gives:

$$
I_{\mathrm{int}}=\int_{0}^{\infty} \frac{\alpha}{\nu^{2}} d \nu=\frac{2 \pi^{2} N f_{i j}\left|\mu_{i j}\right|^{2}}{3 c \epsilon_{0} k T},
$$

and this condition is fulfilled by the result of the theory of $\mathrm{VV}-\mathrm{W}$.

The index of refraction is expressed (see (2.36)) by the sum of two terms: the first term represents the dipole contribution (the contribution of the permanent moments of the molecule); the second term $\bar{n}$ which is added in (2.36), although it does not come out of (2.28), takes into account the contribution of induced polarization and we will have (sec. 2.1) $\bar{n}-1=\left(C^{\iota e}\right) \cdot p_{i}$. It can be shown that the first term $\left(N / 3 \epsilon_{0} k T\right) \sum_{i j} f_{i j}\left|\mu_{i j}\right|^{2} G_{i j}$ reduces, under appropriate conditions, to the dipole term $(1 / 2) .\left(N_{A} p_{i} / R T\right)\left(\mu^{2} / 3 \epsilon_{0} k T\right)$ of the Debye-Langevin relation (see sec. $2.1 \mathrm{~B}$ ). It is sufficient to notice that for the "static" case $(\nu \rightarrow 0)$ we have (2.38) $G_{i j}=1$ and that $N=N_{A} p_{i} / R T$ (2.3) along with $\left.\sum_{i j} f_{i j}\left|\mu_{i j}\right|^{2}=1 / 2\right) \mu^{2} \quad$ [Birnbaum, 1953; Townes and Schalow, 1955; Van Vleck, 1932].

B. Line with parameter $(\Delta \nu)$-The parameter $\Delta \nu$ (which determines the line shape) must be considered, according to the VV-W theory, as an empirical parameter. Its knowledge is required if one wants to use (2.33) to (2.38) for numerical applications. The following considerations are intended to give the reader a more precise understanding of this fundamental parameter.

If we anticipate what will be said in the discussion of the absorption formula, we then recognize that for the case of a narrow absorption line $(\Delta \nu)_{i j}$ represents the half width of the line at the half intensity points. This interpretation is not valid for sufficiently broad lines when the gas pressure is increased. Although then $\Delta \nu$ loses its precise meaning of half width it still retains the meaning of a line width parameter (or "impact parameter," sometimes called half width or half-half width).

This parameter is introduced into the $\mathrm{VV}-\mathrm{W}$ formula to express the ratio $1 / 2 \pi \tau ; \tau$ is the time required by the molecule to cover a distance equal to its mean free path. One can use the methods of the kinetic theory of gases (2.38) while delaying until later the justification of its use.

According to the kinetic theory of gases (molecules are treated as small rigid elastic spheres) and if the velocity distribution function of the molecule is isotropic and Maxwellian, the mean free path $l$ is given by:

$$
l=1 / \sqrt{2} N \sigma,
$$

where $\sigma$ is the collisional cross section:

$$
\sigma=\pi b^{2},(b \text { the effective diameter of shocks) }
$$

and $N$ the number of molecules per unit of volume. 
We have on the other hand:

$$
l=\tau \bar{v}_{1},
$$

where $\bar{v}_{1}$ is the molecules mean velocity with respect to the laboratory coordinates system. The following formulas have been established in kinetic theory:

$$
\bar{W}=(1 / 2) M \bar{V}_{1}^{2}=(3 / 2) k T, \bar{V}_{1}=\sqrt{3 k T / M}, \bar{V}_{11}=\sqrt{2} \bar{V}_{1},
$$

$$
\bar{v}_{1}=\sqrt{8 / 3 \pi} \cdot \bar{V}_{1}=\sqrt{8 k T / \pi M}, \bar{v}_{11}=\sqrt{2} \bar{v}_{1}
$$

$\bar{W}$ is the mean kinetic energy, $M$ the molecular mass in kilograms, $\bar{V}_{1}$ the root mean square of velocity with respect to the laboratory coordinates system and $\bar{V}_{11}$ and $\bar{v}_{11}$ the actual velocity and the mean velocity of a molecule with respect to another (we consider only the case of a pure gas; an example for a mixture of gas will be given in sec. $3.2 \mathrm{~F}$ ).

Recalling the well-known relation, we will have

$$
\Delta \nu=\frac{1}{2 \pi \tau}=N \frac{\bar{v}_{1}}{\sqrt{2}} b^{2}=N \frac{\bar{v}_{11}}{2} b^{2}
$$

for a given temperature, $\bar{v}$ and $b$ are constants while $N$ is proportional to the gas pressure. Consequently, $\Delta \nu$ is also proportional to the pressure.

This simple relation of proportionality $(\Delta \nu \sim p)$ has been experimentally verified in the pressure domain from a few hundredths of tenths of $\mathrm{mm} \mathrm{Hg}$ to a fraction of an atmosphere and sometimes to an atmosphere itself. Departures become more frequent as the pressure is increased. It has been established, for instance, that for ammonia, the parameter $\Delta \nu$ increases more slowly than the pressure when the pressure is above $150 \mathrm{~mm} \mathrm{Ho}$ (sec. 3.2C).

As one would expect, the hypothesis and methods used in kinetic theory should not be valid in such a large domain. This is due to the fact that in kinetic theory one does not take into account the exact nature of the molecular interactions which, in the hertzian domain, determines the cross sections. Generally, the values of cross sections predicted by the kinetic theory are smaller than the observed ones. A more accurate evaluation of the impact parameter $\Delta \nu$ and of the cross sections has been attempted in several theories of line-broadening which will be briefly discussed in section 2.4. We quote the following references: Anderson [1949c and 1950]; Bleaney and Penrose [1947a and 1948b]; Hill and Smith [1950]; Howard and Smith [1950a]; Margenau [1949a and 1949b]; Mizushima [1951a and 1951b]; Smith [1956]; and, Smith and Howard [1950].

C. Discussion of the $V V-W$ absorption formula (2.33) - The formulas (2.33) and (2.36) which resume the results of the $\mathrm{VV}-\mathrm{W}$ theory [1945] have been independently derived by Fröhlich [1946] using a calculus of difference method. Starting with the same hypotheses, Karplus, and Schwinger [1948] have reproduced the same results by using quantum mechanical methods without appealing to the correspondence principle. The VV-W theory, checked once more by Van Vleck and Margenau [1949], seems thus well-established within conditions for which it has been formulated. Experience lends a solid justification to this theory while suggesting some reservations to be mentioned later.

We are going to underline the essential characteristics of this phenomenon of absorption and, in particular, its dependence on frequency, pressure, and gas temperature. The influence of temperature manifests itself in a very complicated fashion. Thus it seems advisable to isolate the effect of temperature which we considered at first as a constant; we will come back to this later in the discussion of results (sec. 3.2D).

This discussion on the effect of frequency and pressure will be conducted, under the assumption that the resonant frequencies, $\nu_{i j}$, are generally independent of the gas pressure $p$ while the line width parameters $(\Delta \nu)_{i j}$ are directly proportional to the pressure. The limitation of these hypotheses for certain cases will require some modification which will be mentioned for the case of ammonia (sec. 3.2). Finally, we will assume that the pressure is not too high so that $(\Delta \nu)_{i j}$ is not larger than a few tenths of $\nu_{i j}$.

One can then write the fundamental eq (2.33) with the help of the dimensionless parameters:

$$
\delta=(\Delta \nu)_{i j} / \nu_{i j} \text { and } x=\nu / \nu_{i j}
$$

and making $\left(N=N_{A} p_{i} / R T\right.$, see $\left.(2.3)\right)$ :

$$
A_{p}=A_{T} p_{i}, \text { with } A_{T}=\frac{2 \pi N_{A}}{3 c \epsilon_{0} k R T^{2}} .
$$

We then have, when we consider the effect of one line assumed "isolated" (examples where the absorption results from the contributions of several lines are treated in sec. 3.2$)$ :

$$
\alpha_{i j}=A_{i j} x^{2} F_{i j} p_{i}=A_{i j} x^{2} \delta\left(\frac{1}{(1-x)^{2}+\delta^{2}}+\frac{1}{(1+x)^{2}+\delta^{2}}\right) p_{i},
$$

with

$$
\begin{gathered}
A_{i j}=A_{T .} f_{i j}\left|\mu_{i j}\right|^{2} \nu_{i j}, \\
F_{i j}=\delta\left(\frac{1}{(1-x)^{2}+\delta^{2}}+\frac{1}{(1+x)^{2}+\delta^{2}}\right)
\end{gathered} .
$$

In (2.49) which expresses the absorption, $A_{i j}$ is a constant factor (for a fixed temperature and a given line), $x$ is proportional to the frequency selected for the measurement, and the pressure $p$ appears explicitly and implicitly through the mean shape factor $F_{i j}$. This factor contains the damping constant $\delta$ assumed proportional to the pressure. Let us 
notice that the expression (2.51) allows for the presentation of the shape factor under the form of nomograms which could be very useful when one seeks a general view of the phenomenon of absorption. The following conclusions are somewhat obvious:

1. If the pressure is fixed and not too small, the general shape of a line is definitely asymmetric. This asymmetry appears on the example of figure 17 (see also sec. 3.2 ).

2. In the neighborhood of the resonant frequency and if the pressure is not too high, we will have

$$
(1-x)^{2}+\delta^{2} \ll(1+x)^{2}+\delta^{2},
$$

and (2.49) can be written:

$$
\alpha_{i j}=A_{i j} x^{2} \frac{\delta}{(1-x)^{2}+\delta^{2}} p_{\imath},
$$

which is typical of resonance absorption.

The absorption becomes maximum when $\left(\alpha_{i j}\right)^{\prime} \nu=0$ and this gives:

$$
\left(\alpha_{i j}\right)_{\max }=A_{i j} \frac{1+\delta^{2}}{\delta} p_{i},
$$

when

$$
x=x_{\max }^{\alpha}=1+\delta^{2} .
$$

This result can be further simplified if one supposes that besides condition (2.52) one has a gas pressure so weak that:

We then have

$$
\delta^{2} \ll 1 \text {. }
$$

$$
\left(\alpha_{i j}\right)_{\max }=A_{i j}\left(p_{i} / \delta\right), \text { for } x=x_{\max }^{\alpha}=1,
$$

and it is only in this case that the absorption maximum is located at the resonance frequency $\left(\nu=\nu_{i j}\right)$. In that case the absorption maximum is independent of pressure. There exists a number of experimental verifications [Birnbaum, 1953; Bleaney and Penrose, 1948a; Townes, 1946].

3. Away from the resonance frequency and for moderate pressures, we have

$$
\delta^{2} \ll(1-x)^{2},
$$

and the absorption in the line wings is (2.49):

$$
\left(\alpha_{i j}\right)_{\mathrm{wing}}=A_{i j} x^{2} \delta\left(\frac{1}{(1-x)^{2}}+\frac{1}{(1+x)^{2}}\right) p_{i} ;
$$

if $\delta$ is assumed proportional to the pressure, the absorption appears proportional to $p_{i}^{2}$ at a given frequency:

$$
\left(\alpha_{i j}\right)_{\mathrm{wing}}=(C t e) \cdot p_{i}^{2} .
$$

Several experimental verifications confirm this result (sec. 3.2) [Battaglia, Bruin, and Gozzini, 1958; Birnbaum, Kryder, and Lyons, 1951; Birnbaum and Maryott, 1953a and 1953b].

If one goes further away from the resonance frequency in the direction of the higher frequency so that:

$$
\delta^{2} \ll 1 \ll x,
$$

eq (2.49) becomes:

$$
\left(\alpha_{i j}\right)_{H . F}=2 A_{i j} \delta p_{i} .
$$

Then the absorption, while still proportional to $p_{i}^{2}$, becomes independent of frequency [Bleaney and Loubser, 1950 ; Loubser, Klein, and Townes, 1949] . . . It would be false to extrapolate this conclusion of optical frequencies where the $\mathrm{VV}-\mathrm{W}$ theory ceases to be valid.

4. For the particular case where the resonance frequencies are very low $(x \gg 1),(2.49),(2.33)$, and (2.36) give :

$\left.\begin{array}{l}\alpha=2 A_{i j} p_{i} \frac{x^{2} \delta}{x^{2}+\delta^{2}}=\frac{4 \pi f_{i j}\left|\mu_{i j}\right|^{2}}{3 c \epsilon_{0} k T} N \frac{\nu^{2}(\Delta \nu)}{\nu^{2}+(\Delta \nu)^{2}}, \\ n-1=\frac{A_{i j} c}{2 \pi \nu_{i j}} p_{i} \frac{\delta^{2}}{x^{2}+\delta^{2}}+\bar{n}=\frac{f_{i j}\left|\mu_{i j}\right|^{2}}{3 \epsilon_{0} k T} N \frac{(\Delta \nu)^{2}}{\nu^{2}+(\Delta \nu)^{2}}+\bar{n}\end{array}\right\}$

One sees that for the limiting case $\nu_{i j}=0$, the VV-W formulas become of the nonresonant type, that is, reduced to the Debye formulas (2.36) where $2 \pi \tau=1$ ) $\Delta \nu$, and where the constant factors are different.

Keeping the same condition and adding $\nu \ll(\Delta \nu)_{i j}$ so that $\nu_{i j} \ll \nu \ll(\Delta \nu)_{i j}$ we find that if the frequency of measurement is sufficiently low and the gas pressure sufficiently high, the absorption becomes independent of the pressure while the index of refraction is proportional to it. Absorption of the Debye type has been indeed observed for some gases. Birnbaum and Maryott [1953b], for instance, have observed that $\mathrm{NH}_{3}$ absorption passed from the resonant type to the nonresonant type in the neighborhood of $90 \mathrm{~mm} \mathrm{Hg}$ while the resonance frequency of the unified line of ammonia (sec. 3.2) becomes practically zero at about $2 \mathrm{~atm}$ [Bleaney and Loubser, 1948 and 1950].

C. Anomalous dispersion-We now consider the VV-W formula for the index of refraction (2.36). With the same substitution as before we write for an "isolated" line:

$$
n-1-\bar{n}=\left(A_{i j} c / 2 \pi \nu_{i j}\right) p_{i} G_{i j},
$$

with

$$
\begin{array}{r}
G_{i j}=\frac{1}{1-x^{2}}\left[1-\frac{x \delta^{2}}{2}\left(\frac{1+x}{(1-x)^{2}+\delta^{2}}-\frac{1-x}{(1+x)^{2}+\delta^{2}}\right)[\right. \\
\equiv \frac{1}{2}\left[\frac{1+x+\delta^{2}}{(1+x)^{2}+\delta^{2}}+\frac{1-x+\delta^{2}}{(1-x)^{2}+\delta^{2}}\right] .
\end{array}
$$

The presence in (2.65) of the factor $A_{i j}$ which appears in the evaluation of the absorption underlines the connection between the phenomena of absorption and dispersion. This connection could not have appeared for the case of normal dispersion (sec. 2.2B).

1. In the neighborhood of resonance frequency, 
when conditions (2.52) and (2.56) are fulfilled, we have

$$
n-1-\bar{n}=\frac{\alpha_{i j} c}{2 \pi \nu_{i j}} \frac{1-x}{x^{2} \delta}=\frac{\left(\alpha_{i j}\right)_{\max } c}{2 \pi \nu_{i j}} \frac{(1-x) \delta}{(1-x)^{2}+\delta^{2}}
$$

$\alpha_{i j}$ and $\left(\alpha_{i j}\right)_{\max }$ being expressed by relations $(2.53)$ and (2.57).

Starting with this last relation, information concerning the connection between the absorption and dispersion can be gathered by means of a classical analysis. Results are displayed in figure 17 which gives a picture of the phenomenon known as anomalous dispersion.

From (2.67), one easily deduces that, under the accepted conditions, the dispersion $\partial n / \partial \nu$ is inversely proportional to the gas pressure at resonance frequency.

The type of interdependence between absorption and dispersion described by the $\mathrm{VV}-\mathrm{W}$ theory constitutes only a particular case of the general relations of Kramers-Kroning [Kerr, 1951].

2 . In the wing of a line and far enough from line center so that $\delta^{2} \ll(1-x)^{2}$ (condition (2.58)), eq (2.65) becomes:

$$
n-1-\bar{n}=\frac{A_{i j} c}{2 \pi \nu_{i j}} p_{i} \frac{1}{1-x^{2}}\left[1-\frac{x \delta^{2}}{2}\left(\frac{1+x}{(1-x)^{2}}-\frac{1-x}{(1+x)^{2}}\right)\right] .
$$

This relation, where $\delta$ and $\bar{n}$ are assumed proportional to the pressure, represents the way in which the index of refraction depends on the gas pressure in the neighborhood of an absorption line (for a given frequency of measurement and for fixed temperature). One notes that for sufficiently weak pressures the slope $\partial(n-1) / \partial p$, instead of remaining constant (sec. 2.1), increases with the pressure if $x>1\left(\nu>\nu_{i j}\right)$ and decreases if $\nu<\nu_{i j}$.

The experimental results concerning this dispersion effect and concerning their comparison with the absorption are rather rare. We shall come back to this when we discuss the results in section $3.2 \mathrm{E}$.

D. The validity of the $V V-W$ theory - The formulas of $\mathrm{VV}-\mathrm{W}$ have been the object of several works of experimental verification, although almost all of these were concerned with the phenomenon of absorption. Several positive verifications bearing on the essential characters of the absorption have already been mentioned above (see sec. 2.3B). The list of these will be more or less completed here to allow us to underline the weak points of the theory in the domain of average pressures in particular (see also sec. 3.2). Numerous are the studies concerning: oxygen [Carrara, 1956; Tinkham and Strandberg, 1955; Van Vleck, 1947]. Measurements by Beringer [1945 and 1946] at $60 \mathrm{Gc} / \mathrm{s}$ have shown that the absorption measured in the line wings is superior to what the theory of $\mathrm{VV}-\mathrm{W}$ had predicted. An explanation has been attempted by invoking the fact that the fine structure of oxygen is not completely hidden at atmospheric pressure [Beringer and Castle, 1951; Strandberg, Meng, and Ingersoll, 1949]. Artman [1953] has shown good agreement between his results and theory.

An absorption stronger than predicted by theory in the high frequency wing has been observed by Becker and Autler [1946] for water vapor in air (measurement made in the band 15 to $45 \mathrm{Gc} / \mathrm{s}$ ). However, a comparison between several experimental results relating to the same question indicates that experimental errors could be considerable.

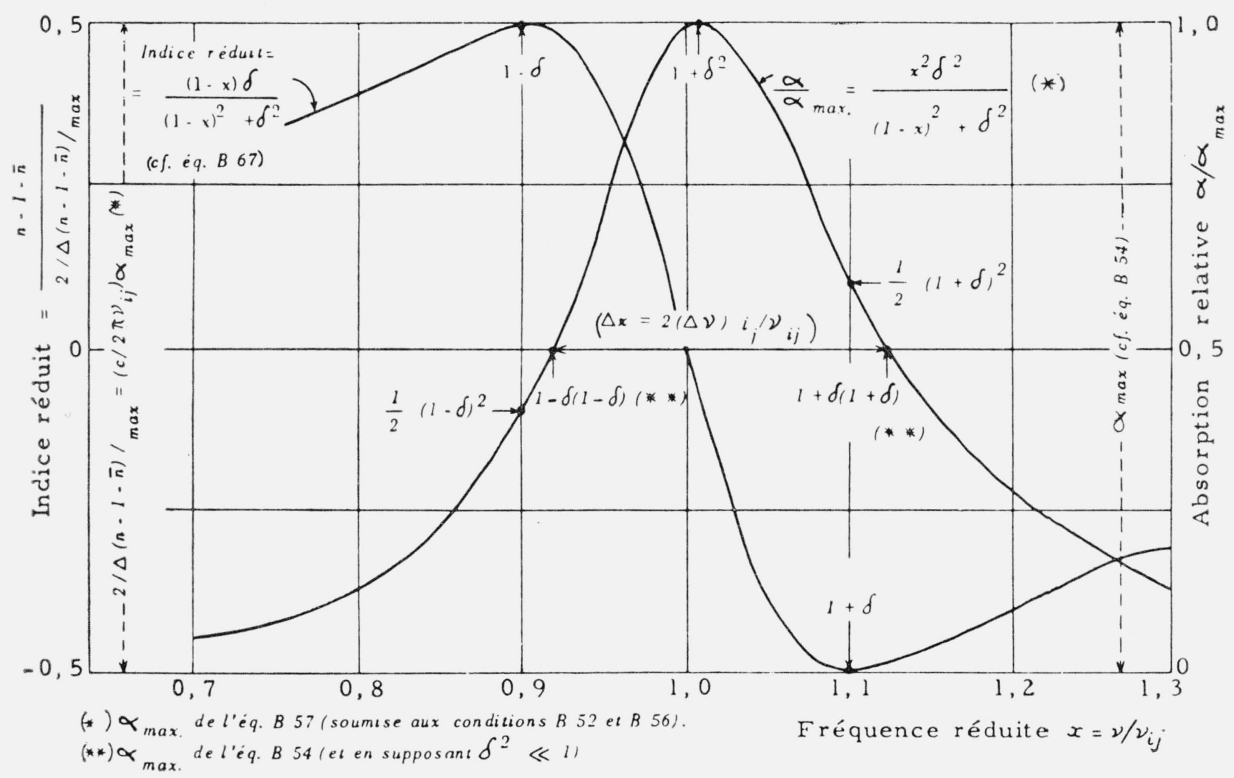

FiguRE 17. Discussion of the interdependence between absorption and dispersion (anomalous dispersion). 
Values calculated for the absorption are also affected by the uncertainty on some of the quantities which are needed for its computation, notably $\Delta \nu$ (which varies from one line to the next).

Similarly large departures have been observed by Weidner [1948] on IC1 measurement and they seem to suggest that under certain circumstances the validity of the $\mathrm{VV}-\mathrm{W}$ theory is rather doubtful, while in general its foundation is basically sound. The weakness of the absorption predicted by the theory in the wings of the unified line of ammonia, seem again to be confirmed (sec. 3.2). Finally, we must admit that generally the $\mathrm{VV}-\mathrm{W}$ theory tends to predict, for frequencies far from the resonance frequencies, a weak absorption (sometimes twice to three times smaller than the measured values).

No proper corrective formulas have been proposed so far, and the $\mathrm{VV}-\mathrm{W}$ formulas continue to be the main theoretical tool in the domain where they apply.

\subsection{Other Theories of Collisional Broadening}

Anderson [1949a and 1949c]; Artman [1953]; Artman and Gordon [1954]; Lorentz [1906 and 1909]; Margenau [1935, 1949a and 1949b]; Margenau and Bloom [1959], Mizushima [1951a and 1951b]; and, Townes and Schalow [1955].

The weak point of the $\mathrm{VV}-\mathrm{W}$ theory is the fact that the line broadening parameter $\Delta \nu$ is considered as an essentially empirical one (sec. 2.3B). A more complete theory should be capable to evaluate this parameter by making use of intermolecular forces (Van der Waals' forces).

The general formulation of the problem presents no difficulties. In trying to give the problem a general solution, Jablonski $[1940,1945,1946$, and 1948] treats the ensemble of the gas as a system, a kind of large unique molecule, inside of which transition takes place between a multitude of levels. The mathematical difficulty quickly becomes very serious and if one wishes to arrive at useful results, simplification and approximations are necessary. It is at this time that the several collision theories and statistical theories have been deveoloped.

The problem which is the object of these theories is the one of line broadening by collision (determination of line shapes and width parameters). Collisional broadening is by far the most important cause of line broadening in the microwave spectroscopy of gases. Other causes are present at weak pressures or under special conditions. They are natural broadening, Doppler broadening, broadening through collisions with the container's wall, broadening by Zeeman and Stark effects, and broadening through saturation. We shall not worry about these. The fact that for average pressure, and if one stays away from saturation effect, collisional broadening is the most important one, and renders microwave spectroscopy the most fruitful field for the study of this kind of broadening. Consequently, important conclusions may be made on molecular structure and interaction.
A. Collisional theories - In these theories it is generally admitted that the molecules are sufficien tly distant from each other so that they can be considered as isolated. The molecular interactions at the time of "collisions" are responsible for the perturbations of the energy levels and for the broadening of the absorption lines. But since the absorption during impact is usually neglected, the validity of these theories becomes somewhat doubtful for higher pressures (a few atmospheres). Their validity is also somewhat limited by some of the simplifying hypotheses of the kind mentioned in the $\mathrm{VV}-\mathrm{W}$ theory (sec. 2.3A).

Collisional theories belong usually (with the exception of Anderson's theory [1949a and 1949c]), to the adiabatic type. That is, the phase of the molecular oscillator is perturbed without affecting the energy. Let us recall, for instance, that it is only after passing. to quantum mechanics that the idea of transition is introduced in the $\mathrm{VV}-\mathrm{W}$ theory. It appears, however, that contrary to what happens in the optical domain, collisions in the hertzian domain are almost always "diabatic."

Lorentz [1906] was the first one who, starting with older concepts yet, has formulated a theory for collision broadening of lines in the optical domain. One could reproduce his result by using the method (sec. 2.3) in which one would suppose that the initial position and velocities of the molecular oscillators are distributed at random immediately following impact $\left\langle\left\langle s\left(t_{0}\right)\right\rangle=0\right.$ and $\left.\left\langle v\left(t_{3}\right)\right\rangle=0\right)$. Bruin [1956] develops, in his thesis, a generalized method of computation where the old friction model of Helmholtz and the collisional models of Lorentz and VV-W appear as particular cases.

Before the publication of the $\mathrm{VV}-\mathrm{W}$ theory, Weisskopf [1932] investigated carefully the process of collision by means of Fourier integrals. This method was followed by other theories: Anderson [1949a and 1949c]; Leslie [1951]; Mizushima [1951a and 1951b]; and many others [Foley, 1942 and 1946; Lenz, 1933; Lindholm, 1941, 1942, and 1945].

Among all the theories of collision broadening, that of Anderson's appears to be the most complete, it also benefits from experimental verifications [Anderson, 1949b and 1950; Artman, 1953; Artman and Gordon, 1954].

B. Statistical theories-Around 1934-1937 another type of collisional broadening theory was developed. It is seen mostly in the work of Kuhn [1934 and 1937], Kuhn and London [1934], in the work of Margenau [1935], Margenau and Watson [1936], and other workers (see [Ishiguro, Arai, Mizushima, and Kotani, 1952]). Under the statistical approach each molecule is considered under the permanent perturbing inference of all the other molecules (one could also take into account the effect of multiple collisions). Although this general hypothesis seems justified, the statistical theories take on a statistical character by admitting that molecules are almost motionless. They deprive themselves of the possibility of taking into account molecular interactions. An improved version of Margenau's theory [1949a and 1949b] does 
interpret correctly the experimental results of ammonia measurements.

It can be shown generally that the statistical theories reduce to collisional theories as soon as the collisions are sufficiently slow or when the measured frequency is sufficiently far from the resonant frequency [Holstein, 1950; Margenau and Bloom, 1950]. Discussions on the possibility of a synthesis of the two types of theories as well as on their validity continue: Anderson [1952], and Margenau [1956]. . .

The comparison of the several available theories of collisional broadening with experimental results shows that agreement is rather rare (the dipoledipole interaction of ammonia being the only exception). The causes seem to be many: cases where only one kind of molecular interaction dominates are infrequent; some of the molecular constants to which theories are appealing are unknown or poorly known; experimental results are often contradictory.

\section{Results and Discussions}

\subsection{Index of Refraction of Atmospheric Gases, Water Vapor, and Air}

A. Introduction-Recent developments in microwave techniques have furnished a new means of measuring fundamental physical quantities, such as the speed of propagation of electromagnetic waves and the index of refraction. The precision obtained is comparable, sometimes superior, to that reached by the classical means of optics.

Knowledge of the index of refraction of the atmosphere and of the speed of propagation allows, to quote only one practical application, the determination of distances by means of electromagnetic detection (radar). The velocity of propagation in vacuum has been measured recently in the hyperfrequency domain by several workers whose work has been resumed in the very interesting article by Froome [1956]. The value given by him is $299.793 \times 10^{8} \pm 300 \mu$ sec. The precision on the index of refraction measured by hertzian method in the works of Crain, Birnbaum, Essen and Froome, and Gozzini, is in the best cases of the order of a few parts in ten thousand, but it often falls to a few parts in a thousand. Previous determinations in the low frequency bands $(1 \mathrm{Mc} / \mathrm{s})$ suffer from a much larger dispersion [Hector and Woernley, 1946]. This discrepancy could have been caused principally by systematic errors introduced through parasitical circuits, poorly known in those days, and as far as water vapor is concerned, by the high sensitivity of isolating supports and condenser plates to adsorption. The hyperfrequency domain where the resonant cavity technique shows itself to be far superior, constitutes a favorable domain and because of the desired precision it is not without interest to dwell on measurements of the index of the atmosphere. Let us note that the principle of measurement proposed by Gozzini which we have used, seems to lead to a precision slightly superior to that obtained by Birnbaum, etc., without requiring quartz fre quency standards such as those used in the EssenFroome experiment. Finally, we wanted to extend these measurements to all the atmospheric constituents and, consequently, the measurements on rare gases (krypton, neon, and xenon) are, to our knowledge, original.

B. Experimental conditions-Measurements have been made at fixed frequencies between 7 and 12 Gc/s (klystrons $723 \mathrm{~A} / \mathrm{B}$ and X $13 \mathrm{~B} /$ Varian) and with the gas pressure of the measurement cavity decreasing progressively from $1 \mathrm{~atm}$ to a fraction of 1 $\mathrm{mm} \mathrm{Hg}$ (except for water vapor). Each series of measurements produced a curve $(n, p)$ determined by a few tenths of points and for each gas several series of measurements have been repeated ( 5 to 10 times). The gas temperature is maintained within $\pm 0.1{ }^{\circ} \mathrm{C}$ between $0{ }^{\circ} \mathrm{C}$ and $50{ }^{\circ} \mathrm{C}$. The sensitivity used corresponded to a few volts of triangular tension (top value) applied to the klystron reflector. The instrument was frequently calibrated with respect to argon for which the sensitivity used corresponded to $\mu$ secs per $\mathrm{mm} \mathrm{Hg}$. The index of argon has been measured by means of absolute calibration (ch. 1).

The limitation on the precision was caused more by the difficulty of reading the pressure than by the electronic components. The reading of the barometric gauge has been improved in the case of water vapor by the usage of a cathetometric reader.

C. Dry air and its constituents - Atmospheric gases have been collected into bottles or vials purchased from commercial firms. Results have been corrected for the principal impurities mentioned by the manufacturers. These corrections are usually smaller than the probable error. Those gases are dehydrated by sending them through a succession of tubes containing $\mathrm{CaSO}_{4}$ and $\mathrm{P}_{2} \mathrm{O}_{5}$ prior to the filling of the measuring cavities. The dehydrating process used for atmospheric air was a bit more involved, a cold trap of solid $\mathrm{CO}_{2}$ was added. Other processes to dry the air have been tried or utilized such as those making use of concentrated sulfuric acid and anhydrous sodium carbonate. Small traces of humidity will result in a higher measured value of the index of refraction. This question has been well discussed by Watson et al., [1931 and 1934]. A reproducible measure of the minimum value of the index of refraction constitutes the best criterion insuring that the sample has been well dehydrated.

The general results of those measurements are indicated on figure 18 . One notices that the dependence of the index of refraction on the gas pressure is, in all cases, linear, which is in accordance with the Clausius-Mosotti law for nonpolar gases (sec. 2.1). The variation of the index of refraction with the inverse of the absolute temperature, also predicted by this law, has been equally verified, although these results are not indicated on figure 18 so as not to clutter the drawing.

Table 1 resumes the results, after the Van der Waals correction for real gases, for normal temperature and pressure (NTP) $\left(0^{\circ} \mathrm{C} 760 \mathrm{~mm} \mathrm{Hg}\right)$. Our 


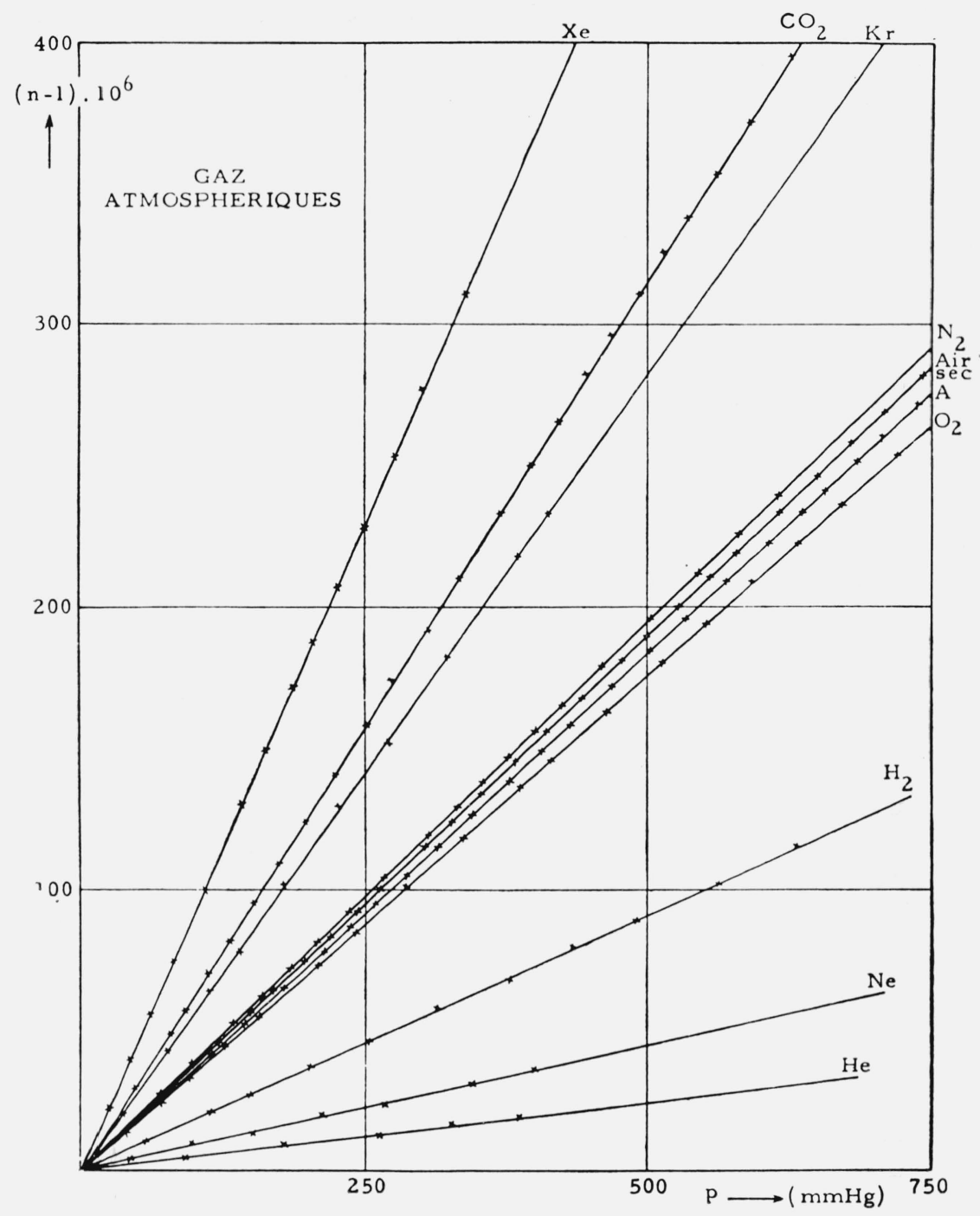

FIGURE 18. Index of refraction of atmospheric gases and dry air as a function of the pressure in the centimetric waves domain $\left(\mathrm{t}=0^{\circ} \mathrm{C}\right)$

results with an indication of the probable error for each gas are presented along with results of other authors, in order to give a more complete picture of the present day situation of the problem. An average value of the principal results is given in the domain of hyperfrequencies for each gas. These averages have been weighted in proportion to the inverse of the square of the probable error.

The results in the hyperfrequency domain can be compared with those obtained for low frequencies (around $1 \mathrm{Mc} / \mathrm{s}$ ) or with the results in the optical domain. The present table is concerned with the totality of these results. In the first case, the results should be equivalent as long as the frequency is sufficiently lower than $60 \mathrm{Gc} / \mathrm{s}$ where an absorption band of oxygen is located. One notes discrepancies. With the exception of air and oxygen at $72 \mathrm{Gc} / \mathrm{s}$ these discrepancies are caused by experimental error and not by an abnormal dispersion effect (sec. 2.3C).
Such an effect manifests itself only for oxygen at 72 $\mathrm{Gc} / \mathrm{s}$ and then remains small as predicted by Van Vleck [Kerr, 1951]. Measurements in the optical frequency range are located on the other side of these absorption bands and are not generally directly comparable with measurements in the hyperfrequency range unless one extrapolates for infinite wavelengths [Barrell, 1951].

The paramagnetic property of oxygen (which is in fact responsible for the microwave absorption of that gas) makes it unrealistic to apply the simplifying relation:

$$
\epsilon_{r}=n^{2}
$$

between the dielectric constant and the index of refraction. One must use the exact relation:

$$
\mu_{\tau} \epsilon_{r}=n^{2}
$$


TABLE 1. Results of Measurements of the Index of Refraction of Atmospheric gases (without water vapor) at $N T P$ ( $O{ }^{\circ} C$, $760 \mathrm{~mm} \mathrm{Hg}$ )

\begin{tabular}{|c|c|c|c|c|}
\hline Date & Author & $(n-1) \cdot 10^{6}$ & $\left(\epsilon_{r}-1\right) \cdot 10^{6}$ & $\begin{array}{c}\text { Frequency } \\
(\mathrm{Mc} / \mathrm{s})\end{array}$ \\
\hline
\end{tabular}

1-Dry air (287.98)

\begin{tabular}{|c|c|}
\hline (1951) & Barrell. \\
\hline 1934 & Watson etc. \\
\hline 1946 & Hector-Woernley \\
\hline 1948 & Crain \\
\hline 1948 & Lyons-Birn.-Kryder \\
\hline 1950 & Philips__. \\
\hline 1951 & Birnbaum-Kr.-Lyons_ \\
\hline 1951 & Essen-Froome_.. \\
\hline 1951 & Gozzini_. \\
\hline 1952 & Zieman $_{---}$ \\
\hline 1952 & Hugues-Armstrong_.. \\
\hline 1953 & Essen \\
\hline 1955 & Froome_- \\
\hline 1955 & Saito _. \\
\hline 1957 & Batt-Boud.- \\
\hline 1958 & Boudouris_. \\
\hline
\end{tabular}

\begin{tabular}{c|}
$287.8 \pm 0.1$ \\
$(288)(\boldsymbol{\beta})$ \\
$(283.5 \pm 0.5)$ \\
$(286)$ \\
$(288.5 \pm 0.5)$ \\
$(299)$ \\
$287.9 \pm 0.7$ \\
$288.15 \pm 0.10$ \\
$(289.3 \pm 1.5)$ \\
$287.9 \pm 1.0$ \\
$(28.5 .5 \pm 2)$ \\
$288.10 \pm 0.1$ \\
$287.66 \pm 0.11$ \\
$(287.0 \pm 1.2)$ \\
$288.0 \pm 0.3$ \\
$288.0 \pm 0.3$ \\
\end{tabular}

$(575.7 \pm 0.2)$
576
$566.9 \pm 1.0$
572.0
$577.0 \pm 1.0$
$598(\lambda)$
$575.4 \pm 1.4$
$576.0 \pm 0.2$
$578.6 \pm 3(\delta)$
$575.4 \pm 2.1$
$569 \pm 4(\epsilon)$
$575.8 \pm 0.2$
$574.9 \pm 0.22$
$574.0 \pm 2.5$
$575.6 \pm 0.6$
$575.6 \pm 0.6$

$(\alpha)$
0.5
1.0
9000
9000
3000
9000
24000
9000
9000
3000
9000
72000
9000
9000
9000

2-Argon (A) (277. 79)

\begin{tabular}{|c|c|c|c|c|}
\hline 1908 & Burton & 283.7 & $(567.5)$ & $(5890 \dot{\mathrm{A}})$ \\
\hline (1948) & $\begin{array}{l}\text { Burton citê par Kaye- } \\
\text { Laby. }\end{array}$ & 279.2 & (558.5) & $(\alpha)$ \\
\hline 1932 & Cuthbertson & 277.3 & $(554.7)$ & $(\alpha)$ \\
\hline 1934 & Watson-Rao-Ram & $\stackrel{(275)}{(27)}$ & 550 & 0.5 \\
\hline $\begin{array}{l}1946 \\
1948\end{array}$ & Jelatis & $(277.1+0.45)$ & $\begin{array}{l}540.1 \pm 0.0 \\
554.2+0.9\end{array}$ & 1.0 \\
\hline 1949 & Clay-Maesen & $(278 \pm 1)$ & $556+2$ & 1.0 \\
\hline 1951 & Essen-Froome & $277.8 \pm 0.2$ & $555.7 \pm 0.4$ & 24000 \\
\hline 1951 & Michels-Seld.-Over-. & $(299,3)$ & 598,6 & 0.5 \\
\hline 1954 & Heineken-Bruin & $277.0 \pm 1.0$ & $(554.0 \pm 2.0)$ & 25000 \\
\hline 1955 & Froome_ & $277.7 \pm 0.2$ & $(555.4 \pm 0.4)$ & 72000 \\
\hline 1958 & Gozzini-Batt.-Bruin_ & $277.8 \pm 0.1$ & $(555.6 \pm 0.2)$ & 9000 \\
\hline 1958 & Boudouris........ & $277.8 \pm 0.1$ & $(555.6 \pm 0.2)$ & 9000 \\
\hline
\end{tabular}

3-Azote $\left(\mathrm{N}_{2}\right)$ (294.09)

\begin{tabular}{|c|c|c|c|c|}
\hline 1874 & Mascart. & 297.2 & $(594.4)$ & $(5890 \dot{\mathrm{A}})$ \\
\hline (1948) & Scheel citè par Kaye-Laby & 290.6 & $(581.3)$ & \\
\hline 1932 & Michels-Michels & (286) & 573 & 0.5 \\
\hline 1934 & Watson-Rao-Ram_.... & (293.5) & 587 & 0.5 \\
\hline 1946 & Hector-Woernley_ & $(289.8 \pm 0.5)$ & $579.6 \pm 1.0$ & 1. 0 \\
\hline 1948 & Lyons-Birn.-Kryder_ & $293.5 \pm 1.0$ & $587.0 \pm 2.0$ & 9000 \\
\hline 1951 & Birnbaum-Kr.-Lyons & $(293,5 \pm 1,5)(\boldsymbol{\beta})$ & $586.9 \pm 2.9$ & 9000 \\
\hline 1951 & Essen-Froome. & $294.1 \pm 0.1$ & $588.3 \pm 0.2$ & 24000 \\
\hline 1952 & Zieman & $(293.5 \pm 1.0)$ & $587.0 \pm 2.0$ & 9000 \\
\hline 1953 & Essen_. & $294.1 \pm 0.1$ & $(588.2 \pm 0.2)$ & 9000 \\
\hline 1954 & Heineken-Bruin & $\begin{array}{l}294.1 \pm 0.1 \\
294 \pm 1\end{array}$ & $(588 \pm 2)$ & 25000 \\
\hline 1958 & Boudouris & $294.3 \pm 0.5$ & $(588.6 \pm 1.0)$ & 9000 \\
\hline
\end{tabular}

4-Gaz Carbonique $\left(\mathrm{CO}_{2}\right)(493.90)$

\begin{tabular}{|c|c|c|c|c|}
\hline $\begin{array}{l}1927 \\
(1952)\end{array}$ & Tausz-Hornug _. & 449.1 & (898. 2) & $(5880 \dot{A})$ \\
\hline $\begin{array}{l}(1952) \\
1934\end{array}$ & $\begin{array}{l}\text { citè Par Guerin } \\
\text { Watson-Rao-Ram }\end{array}$ & $\begin{array}{c}450 \\
(494.5)\end{array}$ & $\begin{array}{l}(900) \\
989\end{array}$ & $(5890 \AA$ \\
\hline $\begin{array}{l}1954 \\
1946\end{array}$ & Hector-Woernley. & $(493.7 \pm 1.0)$ & $\begin{array}{c}989 \\
987.5 \pm 2.0\end{array}$ & $\begin{array}{l}0.5 \\
1.0\end{array}$ \\
\hline 1948 & Lyons-Birn.-Kryder- & $(494.0 \pm 1.0)$ & 988. $0 \pm 2.0$ & 9000 \\
\hline 1949 & Clay-Maesen_. & $(508.5 \pm 1.5)$ & $1017 \pm 3$ & 1.0 \\
\hline 1951 & Birnbaum-Kr.-Lyons. & $(492.7 \pm 1.5)$ & $985.5 \pm 3.0$ & 9000 \\
\hline 1951 & Essen-F roome_... & $494.0 \pm 1.0$ & $988.0 \pm 2.0$ & 24000 \\
\hline 1951 & Gozzini_- & $(495.0 \pm 2.0)$ & $990.0 \pm 4.0$ & 9000 \\
\hline 1952 & Zieman_. & $(493.7 \pm 2.0)$ & $987.5 \pm 4.1$ & 9000 \\
\hline 1954 & Heineken-Bruin & $496 \pm 2$ & $(992 \pm 4)$ & 25000 \\
\hline 1958 & Gozzini-Batt.-Bruin - & $555.4 \pm 0.2(\zeta)$ & $(1110.8)$ & 9000 \\
\hline 1958 & Boudouris . & $493.5+1.0$ & $(987.0+2,0)$ & 9000 \\
\hline
\end{tabular}

(a) Values obtained from optical measurements after extrapolation to infinite wavelength.

which, since $\epsilon_{r}$ and $n$ are closa to unity, can be rewritten as:

$$
\epsilon_{r}-1=2(n-1)-\left(\mu_{r}-1\right)
$$

where $\mu_{r}-1=1.8 \times 10^{6}$ for oxygen and about $0.4 \times 10^{-6}$ for air (containing $21 \%$ of oxygen) at N'TP [Birnbaum, Kryder, and Lyons, 1951]. This correction has been applied to our results as

\begin{tabular}{|c|c|c|c|c|}
\hline Date & Author & $(n-1) \cdot 10^{6}$ & $\left(\epsilon_{1}-1\right) \cdot 10^{6}$ & $\begin{array}{c}\text { Frequency } \\
(\mathrm{Mc} / \mathrm{s}\end{array}$ \\
\hline \multicolumn{5}{|c|}{ 5-Helium (He) (35.06) } \\
\hline $\begin{array}{c}1932 \\
(1952) \\
1946 \\
1948 \\
1951 \\
1953 \\
1958\end{array}$ & $\begin{array}{l}\text { Cuthbertson } \\
\text { cité par Guerin } \\
\text { Hector-Wornley } \\
\text { Jelatis_.... } \\
\text { Birnbaum-Kr-Lyons } \\
\text { Essen } \\
\text { Boudouris_... }\end{array}$ & $\begin{array}{l}34.6 \\
34 \\
(34.2 \pm 0.3) \\
(34.6 \pm 0.1) \\
(35.2 \pm 0.6) \\
35.0 \pm 0.2 \\
35.3 \pm 0.5\end{array}$ & $\begin{array}{c}(69.2) \\
(68) \\
68.4 \pm 0.5 \\
69.2 \pm 0.2 \\
70.5 \pm 1.1 \\
(70.0+0.4) \\
(70.6 \pm 1.0)\end{array}$ & $\begin{array}{c}(\alpha) \\
(5890 \AA) \\
1 \\
1 \\
9000 \\
9000 \\
9000\end{array}$ \\
\hline \multicolumn{5}{|c|}{ 6-Hydrogene $\left(\mathrm{H}_{2}\right)$ (136.16) } \\
\hline $\begin{array}{l}(1952) \\
1951 \\
1953 \\
1956 \\
1958\end{array}$ & $\begin{array}{l}\text { cité par Guerin } \\
\text { Gozzini } \\
\text { Essen } \\
\text { Gozzini-Batt.-Bruin. } \\
\text { Boudouris... }\end{array}$ & $\begin{array}{c}139 \\
(138.8 \pm 1.0) \\
136.0 \pm 0.2 \\
176.7 \pm 0.1(\zeta) \\
137.5 \pm 1.0\end{array}$ & $\begin{array}{l}(278) \\
277.6 \pm 2.0 \\
(272.0 \pm 0.4) \\
(353.4 \pm 0.2) \\
(275.0 \pm 2.0)\end{array}$ & $\begin{array}{c}(5890 \AA) \\
9000 \\
9000 \\
9000 \\
9000\end{array}$ \\
\hline
\end{tabular}

7-Krypton (Kr) (429.0)

\begin{tabular}{|c|c|c|c|c|}
\hline $\begin{array}{l}(1952) \\
1958\end{array}$ & $\begin{array}{l}\text { cité par Guerin } \\
\text { Boudouris... }\end{array}$ & $\begin{array}{c}428 \\
429.0 \pm 2.5\end{array}$ & $\begin{array}{c}(856) \\
(858.0 \pm 5.0)\end{array}$ & $\begin{array}{l}(5890 \AA) \\
9000\end{array}$ \\
\hline \multicolumn{5}{|c|}{ 8-Neon $(\mathrm{Ne})(68.1)$} \\
\hline $\begin{array}{c}(1952) \\
1958\end{array}$ & $\begin{array}{l}\text { cité par Guerin } \\
\text { Boudouris }\end{array}$ & $\begin{array}{c}67 \\
68.1 \pm 0.7\end{array}$ & $\begin{array}{c}(134) \\
(136.2 \pm 1.4)\end{array}$ & $\begin{array}{l}(5890 \AA) \\
9000\end{array}$ \\
\hline
\end{tabular}

9-Oxygene $\left(\mathrm{O}_{2}\right)(266.33)$

\begin{tabular}{|c|c|c|c|c|}
\hline 1908 & Rentschler.- & 271.8 & $(543.6)$ & $(5890 \AA)$ \\
\hline (1948) & $\begin{array}{l}\text { Rentschler cité par Kaye- } \\
\text { Laby }\end{array}$ & & $(532.7)$ & \\
\hline 1934 & Watson-Rao-Ram. & $(265.5)(\beta)$ & 531 & 0.5 \\
\hline 1946 & Hector-Woernley _. & $(261.7 \pm 0.5)$ & 523. $3 \pm 1.0$ & 1.0 \\
\hline 1948 & Jelatis & $(266.3 \pm 0.7)$ & $532.5 \pm 1.3$ & 1.0 \\
\hline 1948 & Crain & $(265)$ & 530 & 9000 \\
\hline 1948 & Lyons-Birn.-Kryder. & $(266 \pm 1)$ & $532 \pm 2.0$ & 9000 \\
\hline 1951 & Birnbaum-Kr.-Lyon & $265.9 \pm 1.0$ & $530 \pm 1.9$ & 9000 \\
\hline 1951 & Essen-Froome. & $266.4 \pm 0.2$ & 531. $0 \pm 0.4$ & 24000 \\
\hline $\begin{array}{l}1951 \\
1952\end{array}$ & Zieman & $\begin{array}{l}200.9 \pm 0.2 \\
266.9 \pm 1.0\end{array}$ & $532.0 \pm 2.1$ & 9000 \\
\hline $\begin{array}{l}1952 \\
1953\end{array}$ & Essen & $\begin{array}{l}200.9 \pm 1.0 \\
266.2 \pm 0.2\end{array}$ & $530.6 \pm 0.4$ & 9000 \\
\hline 1954 & Heineken-Bruin & $268.0 \pm 1.5$ & $534.2 \pm 3.0$ & 25000 \\
\hline 1955 & Froome & $263.9 \pm 0.2$ & $526.0 \pm 0.4$ & 72000 \\
\hline 1958 & Boudouris... & $266.7 \pm 1.0$ & $531.6 \pm 2.0$ & 9000 \\
\hline
\end{tabular}

10-Xenon (Xe) (691.5)

\begin{tabular}{|c|c|c|c|c|}
\hline $\begin{array}{c}(1952) \\
1958\end{array}$ & $\begin{array}{l}\text { Cité par Guerin } \\
\text { Boudouris }\end{array}$ & $\begin{array}{c}704 \\
691.5 \pm 4.5\end{array}$ & $\begin{array}{c}(1408) \\
(1383 \pm 9)\end{array}$ & $\begin{array}{l}(5890 \AA) \\
9000\end{array}$ \\
\hline
\end{tabular}

( $\beta$ ) These values, in parentheses, are deduced from the relation $(n-1)=(1 / 2)$ $\left(\epsilon_{r}-1\right)$. In the case of oxygen and for the case of air, the correction for magnetic permeability is neglected. Let us recall that, although many authors give their results in terms of the quantity $\epsilon_{r}-1$ measurements in the microwave region results in terms of the quantity $\epsilon_{r}-1$ measurement

$(\gamma)$ This high value seems to be caused by the method used by Phillips. The probable error is of the same order of magnitude as the difference with respect to other measurements.

${ }^{(\delta)}$ Measurements reduced to ambient temperature.

(є) The authors discuss improvements of their experimental setup as well as necessary precautions to be taken to obtain a higher accuracy.

(5) The authors mention that these high values could be caused by the presence of impurities.

well as to the measurements of other workers presented in table 1.

As far as hydrogen is concerned, efforts have been made to compute its induced molecular polarization using quantum mechanical techniques (see sec. 2.1). Ishiguro and his colleagues [1952] who have revised and improved this type of computation have found a calculated polarization corresponding to $(n-1)$ $\times 10^{6}=133.3$ at N'TP [Essen, 1953a and 1953b]. 
This theoretical value is roughly 2 percent lower than the measured one, but it is, of course, obvious that the theoretical computation would give a larger value if one could keep a larger number of terms in the wave function of the molecule.

A check on the consistency of our results would be provided by the computation of the index of refraction from the measured values of the indices of its constituents [Vassy, 1956]. Limiting ourselves to the main constituents of air: 78.06 percent nitrogen, 21 percent of oxygen, and 0.94 percent of argon, we find $(n-1) \times 10^{6}=288.3 \pm 0.6$ to be compared with $288.0 \pm 0.3$ measured for dry air.

D. Water vapor The water vapor used came from a container filled with distilled water whose temperature was kept $5^{\circ}$ below that of the measuring cavity (except for measurement at $0{ }^{\circ} \mathrm{C}$ ). The maxima of pressure for nonsaturated vapor were between a few $\mathrm{mm} \mathrm{Hg}\left(0{ }^{\circ} \mathrm{C}\right)$ and $150 \mathrm{~mm} \mathrm{Hg}$ $\left(63^{\circ} \mathrm{C}\right)$. The observed pressure $(p)$ was reduced to the pressure $\left(p_{i}\right)$ corresponding to vapor treated as an ideal gas and obeying the relation:

$$
p_{i}=\left[1-\frac{p}{R T}\left(\frac{a}{R T}-b \cdot p,\right)\right]
$$

$a$ and $b$ are the constants of Van der Waals. The difference between $p$ and $p_{i}$ is of the order of 1 percent under the conditions of our experiment.

A large number of measurements have been made for each of the selected temperatures in the interval $0^{\circ}$ to $63{ }^{\circ} \mathrm{C}$. Measurements at $0^{\circ}$ and $21{ }^{\circ} \mathrm{C}$ have been made at the University of Pisa [Battaglia, Boudouris, and Gozzini, 1957]; they have been completed for other temperatures at the Laboratoire de Physique de l'Atmosphere. The curves obtained for the index $(n-1) \times 10^{6}$ versus pressure at fixed temperature were always straight lines whose slopes are indicated on figure 19. This important effect is contradictory with the results of certain radio frequency measurements where an abnormal dependence of the index on the pressure had been established, but it is in good accord with the hyperfrequency measurement of Birnbaum and Chatterjee $[1952]$.

This anomaly in the radio frequency range is blamed on the water adsorption of the insulating supports of the condensers [Stranathan, 1935]. This influence of adsorption is practically eliminated in the hyperfrequency range when one uses a resonance cavity of sufficiently large dimensions [Becker and Autler, 1946; Birnbaum and Chatterjee, 1952; Froome, 1955]. We have noticed no appreciable effect from the association of water vapor molecules nor have we noticed an effect of anomalous dispersion in the band from 7 to $12 \mathrm{Gc} / \mathrm{s}$ (Saxton [1943, 1944, and 1947] reports an anomalous dispersion in the band from 3 to $18 \mathrm{Mc} / \mathrm{s}$ for water vapor at $100{ }^{\circ} \mathrm{C}$ and $1 \mathrm{~atm})$. The measurements of Essen and Froome at 9 [Essen, 1953a and 1953b], 24 [Essen and Froome, 1951 and 1952], and $72 \mathrm{Gc} / \mathrm{s}$ [Froome, 1955] show but a slight dependence of the index on the frequency. This is in accord with theoretical

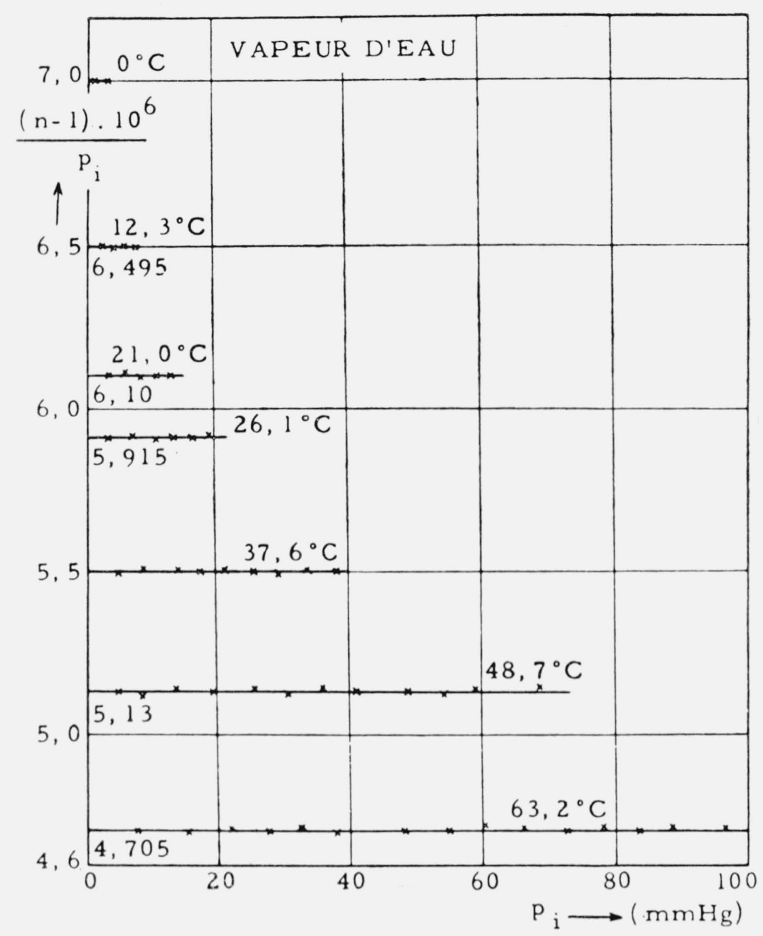

Figure 19. Slope of the index of refraction of water vapor $(\mathrm{n}-1) \times 10^{3} / \mathrm{pi}$ as a function of the pressure and with the temperature as a parameter $(\nu=9300 \mathrm{Mc} / \mathrm{s})$.

predictions which show a weak frequency dependent for the absorption band of water vapor around $300 \mathrm{Gc} / \mathrm{s}(\lambda=1 \mathrm{~mm})$ and a negligible dependence for the band around $22 \mathrm{Gc} / \mathrm{s}(\lambda=1.35 \mathrm{~cm})$ [Kerr, 1951]. One should not expect an anomalous dispersion as long as the frequency remains lower than a hundred $\mathrm{Gc} / \mathrm{s}$.

These measurements lead us to the following expression relating the index of refraction to the pressure and temperature (see sec. 2.1B):

$$
R_{M}=\frac{\epsilon_{r}-1}{\epsilon_{r}+2} \frac{R T}{p_{i}}=A+\frac{B}{T},
$$

$A$ and $B$ are the Debye constants. This relation can be written $\left(R=8.314 \times 10^{3} J /{ }^{\circ} \mathrm{K} \quad M_{k g}, 760 \mathrm{~mm}\right.$ $\left.\mathrm{Hg}=101324 \mathrm{Nw} / \mathrm{m}^{2}\right)$ :

$$
\frac{(n-1) \cdot 10^{6}}{p_{i}}=24.05 \cdot\left(A+\frac{B}{T}\right) \frac{10^{3}}{T}=\frac{K_{2}}{T}+\frac{K_{3}}{T},
$$

where $p_{i}$ is expressed in $\mathrm{mm} \mathrm{Hg}$ and $K_{2}$ and $K_{3}$ are two new constants.

For each measured value of the slope $(n-1) \times$ $10^{6} / p_{i}$ (fig. 18) one deduces $R_{M}=A+B / T$ and this quantity is plotted versus $1 / T$ in figure 19. A curve is then drawn through those points. The method of least squares is used. One obtains the values:

$$
A=(3.99 \pm 0.60) \cdot 10^{-3}, B=20.81 \pm 0.20\left(\mathrm{~m}^{3}\right),
$$

which agree well with the values obtained by other authors [Birnbaum and Chatterjee, 1952]. From 


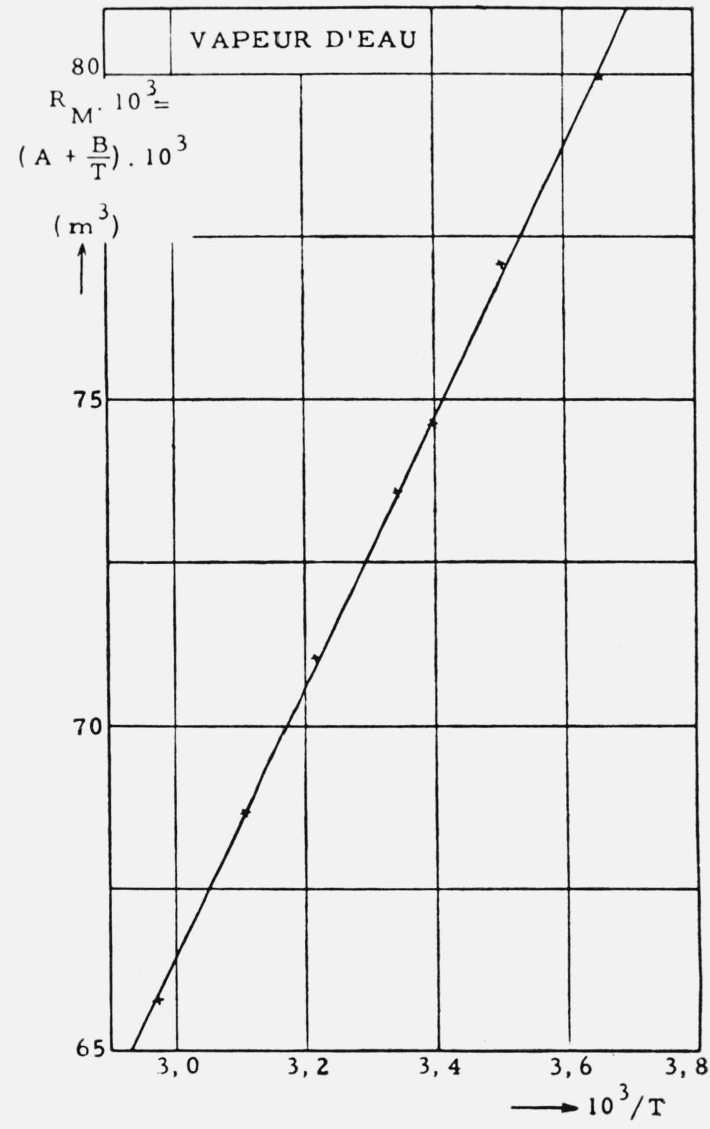

Figure 20. Determination of the constants $A$ and $B$ in the Debye Formula for water vapor.

these values of $A$ and $B$ one obtains:

$$
K_{2}=95.95 \pm 14, K_{3}=(500.5 \pm 4) \cdot 10^{3},
$$

and (3.4) can be written:

$(n-1) \cdot 10^{6}=61.5 \pm 0.8$ to $p=10 \mathrm{~mm} \mathrm{Hg}$ and $t=20^{\circ} \mathrm{C}$.

This last result is compared in table 2 with other results obtained previously.

TABLE 2. Index of refraction of nonsaturated water vapor at $\mathrm{p}=10 \mathrm{~mm} \mathrm{Hg}$ and $\mathrm{t}=20^{\circ} \mathrm{C}$ (average is 60.78)

\begin{tabular}{|c|c|c|c|c|c|}
\hline Date & Author & $(n-1) 10^{6}$ & $\begin{array}{c}\text { Moment dipolaire } \\
\mu \text { (debye) }\end{array}$ & $\begin{array}{l}\text { Fréqu. } \\
(\mathrm{Mc} / \mathrm{s})\end{array}$ & $\begin{array}{l}\text { Tempéra- } \\
\text { ture }\left({ }^{\circ} \mathrm{C}\right)\end{array}$ \\
\hline 1939 & Barrell-Se: & 2. 94 & & $(\alpha)$ & \\
\hline 1930 & Sanger & 62.7 & $1.842 \pm 0.008$ & 1 & (3 points) \\
\hline 1935 & Stranath & $61.3 \pm 0.4$ & & 0.5 & 21-189 \\
\hline 1935 & Groves-Sugden. & $61.6 \pm 1.7$ & $1.845 \pm 0.040$ & & $110-211$ \\
\hline 1942 & Hurdis-Smyth ..... & $61.3 \pm 1.7$ & $1.850 \pm 0.040$ & & $111-249$ \\
\hline 1948 & Crain .... & 61.3 & 1.84 & 9000 & $20-68$ \\
\hline 195 & Phillips. & 62.4 & & 3000 & (amb.) \\
\hline 1951 & Essen-Froome & $607 . \pm 0.1$ & ( $) 1.839 \pm 0.002$ & 24000 & (amb.) \\
\hline 19 & Birnbaum-Chatt_.- & $618 . \pm 0.7$ & ( $) 1.846 \pm 0.005$ & 9000 & 25-103 \\
\hline 195 & Essen & $607 . \pm 0.2$ & ( 1.832 & 9000 & (amb.) \\
\hline 1955 & Froome & $610 . \pm 0.2$ & & 72000 & (amb.) \\
\hline 1957 & $\begin{array}{l}\text { Batt. Boud- } \\
\text { Gozzini }\end{array}$ & 6 & & 9000 & ( 2 points) \\
\hline 1958 & Boudouris....... & $61.5 \pm 0.8$ & $1.848 \pm 0.015$ & 9000 & $0-65$ \\
\hline
\end{tabular}

( $\alpha$ See note of table 1 .

( $)$ The range of temperature variation being small, the authors have taken for the constant $A$ of the Debye formula, a value of the order of 2 .

$(\theta)$ This is an average, taking into account not only the authors' results, but also those of Groves-Sugden, Hurdis-Smyth, and Stranathan.
Once we know the constants $A$ and $B$ we can compute the induced molecular polarization $\alpha$ and the dipole moment $\mu$ of a water vapor molecule (see 2.1B):

$$
A=\frac{\alpha N_{A}}{3 \epsilon_{0}}, B=\frac{\mu^{2} N_{A}}{3 \epsilon_{0} \cdot 3 k}
$$

from which

$$
\left.\begin{array}{l}
\alpha=(1.76 \pm 0.26) \cdot 10^{-40} \frac{C b \cdot m}{V / m} \\
\mu=(6.165 \pm 0.050) \cdot 10^{-30} \mathrm{Cb} \cdot \mathrm{m}
\end{array}\right\}
$$

or :

$$
\left.\begin{array}{l}
\alpha=(52.76 \pm 7.90) \cdot 10^{-12} \frac{\text { debye }}{V / m} \\
\mu=(1.848 \pm 0.015) \text { debye }
\end{array}\right\} \cdot(3.8)
$$

The values obtained by other workers are also given in table 2. Although there is good agreement, a dipole moment of 1.94 debye has been measured using Stark effect at hyperfrequencies [Townes and Schalow, 1955].

E. Damp air - The index of refraction of mixtures of dry air and water vapor can be expressed, according to the hypothesis of additivity of the contributions of the two constituents, by the relation:

$$
(n-1) \cdot 10^{6}=K_{1} \cdot \frac{p_{a}}{T}+K_{2} \cdot \frac{p_{w}}{T}+K_{3} \cdot \frac{p_{w}}{T^{2}}
$$

where $p_{a}$ and $p_{w}$ are the partial pressures of dry air in water vapor $\left(p_{t}=p_{a}+p_{w}, p_{t}\right.$ is the total pressure of the mixture), $K_{2}$ and $K_{3}$ are the constants pertaining to water vapor (see (3.4)) and $K_{1}$ is the constant for dry air. Our results for dry air and water vapor taken separately give:

$$
\begin{array}{r}
K_{1}=103.45 \pm 0.1 \frac{{ }^{\circ} \mathrm{K}}{\mathrm{mm} \mathrm{Hg}}, K_{2}=95.95 \pm 14 \frac{{ }^{\circ} \mathrm{K}}{\mathrm{mm} \mathrm{Hg}} \\
K_{3}=(500.5 \pm 4) \cdot 10^{3} \frac{{ }^{\circ} \mathrm{K}^{2}}{\mathrm{~mm} \mathrm{Hg}} \cdot
\end{array}
$$

Smith and Weintraub [1953a and 1953b] have proposed that the best values to be used in practice should be

$$
\begin{aligned}
K_{1}=103.5 \frac{{ }^{\circ} \mathrm{K}}{\mathrm{mm} \mathrm{Hg}}, K_{2}=96 \frac{{ }^{\circ} \mathrm{K}}{\mathrm{mm} \mathrm{Hg}}, \\
K_{3}=5 \cdot 10^{5} \frac{{ }^{\circ} \mathrm{K}^{2}}{\mathrm{~mm} \mathrm{Hg}}
\end{aligned}
$$

so that

$$
(n-1) \cdot 10^{6}=103.5 \frac{p_{a}}{T}+96 \frac{p_{w}}{T}+5 \cdot 10^{5} \frac{p_{w}}{T}
$$

These resulted from an intensive survey of all previous works in the field. 
Since measurements on dry air are made without $\mathrm{CO}_{2}$, we have increased by 0.01 percent the measured index in order to take into account the presence of 0.03 percent of $\mathrm{CO}_{2}$. (3.12) should be accurate within 0.5 percent in the temperature interval from -50 to $+40{ }^{\circ} \mathrm{C}$, a total pressure between 140 and $760 \mathrm{~mm} \mathrm{Hg}$, a partial pressure of water vapor from 0 to $20 \mathrm{~mm} \mathrm{Hg}$ and for frequencies between 0 and $30 \mathrm{Gc} / \mathrm{s}$.

Because of the obvious advantage of a relation such as (3.12), we have decided to test it. The totality of the result obtained from measurement on dry air made at Pisa [Battaglia, Boudouris, and Gozzini, 1957] from 0 to $21^{\circ} \mathrm{C}$ as well as those made in Paris (1958) are indicated on figure 20. The precision is here limited by the difficulty in preparing mixtures of precise and well-known concentration and sufficient stability. The mixtures are being prepared in separate containers titrated by the partial pressure of water vapor. A precision of 1 to 3 percent on the measured value of the slope $(n-1)$ $\times 10^{6} / p_{t}$ is obtained for mixtures whose partial pressures of water vapor does not exceed 10 percent of the total pressure. The corresponding degree of humidity is already greater than the one usually encountered in the atmosphere. For water vapor content higher than this, the precision is not as good.

Figure 21 proves the validity of (3.12). The lines have been drawn in accordance with this relation while the points have been obtained experimentally. The agreement is very good.

Previous works have suggested slightly higher values for $K_{1}$ and $K_{3}$ and slightly lower value for $K_{2}$ (let us note that in (3.12) the term containing $K_{3}$ is much larger than that one containing $K_{2}$ ). Stickland [1942] had suggested:

$$
\begin{aligned}
K_{1}=105^{\circ} \mathrm{K} / \mathrm{mm} \mathrm{Hg}, K_{2}=90^{\circ} \mathrm{K} / \mathrm{mm} \mathrm{Hg}, \\
K_{3}=5.022 \cdot 10^{5}{ }^{\circ} \mathrm{K}^{2} / \mathrm{mm} \mathrm{Hg} .
\end{aligned}
$$

Saito [1955] has recently made direct measurements on damp air from 0 to $9,000 \mathrm{Mc} / \mathrm{s}$ for water vapor content less than about 2 percent. He finds for the constant $K_{1}$ the value $K_{1}=103$ which is in good agreement with our results. He sees, however, no reason to change the other Stickland coefficient. The preceding discussion justifies, as far as we are concerned, the definitive adoption of relation (3.12) from which the index of refraction of atmospheric air can be computed as soon as the degree of humidity is known.

\subsection{A Study of Absorption and Dispersion in Relation With the Theory of Van Vleck-Weisskopf}

A. Introduction-The formulas of Van VleckWeisskopf (VV-W) constitute the base for the computation made to estimate the absorption and the molecular dispersion of microwaves in the atmosphere (see sec. 2.3 [Anderson, 1949c; Kerr, 1951; Vassy, 1956]) . . . . At present we do not have the proper frequency bands needed for a direct study of atmospheric gases and air $(20$ to $35 \mathrm{Gc} / \mathrm{s}$ for water vapor, 55 to $65 \mathrm{Gc} / \mathrm{s}$ for oxygen), consequently, we have undertaken a research program on the absorption and dispersion of other gases such as ammonia

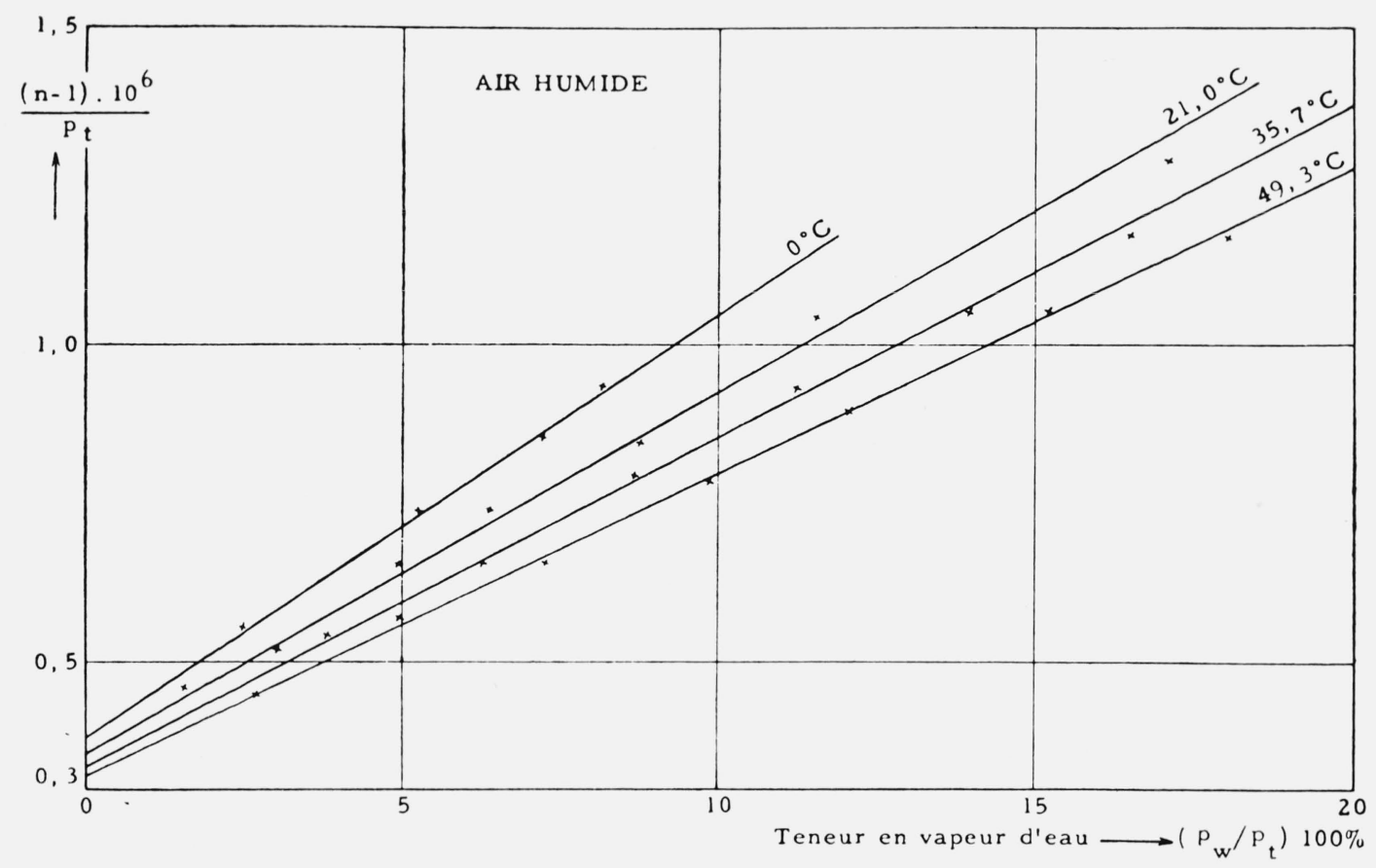

Figure 21. Direct measurements on damp air ( $\mathrm{p}_{\mathrm{t}}$ total pressure of the mixture dry air + water vapor, $p_{u}$ water vapor partial pressure.) 
and chloroform. The results of this study and the establishment of new techniques give us some information of a sufficiently general nature to be useful in the study of the behavior of the atmosphere.

For example, the study of ammonia can be used to approximate the behavior of oxygen. These two gases display hertzian spectra made up of several lines distributed in relatively narrow frequency bands, from this viewpoint a comparison becomes possible. One should bear in mind that in the case of oxygen the absorption is of magnetic origin (interaction between the magnetic moment of the molecule and the magnetic field of the incident electromagnetic wave). Since the magnetic moment is rather weak, the effective cross section, which determines the width of the lines, is very close to the cross section computed in the Kinetic Theory of Gases. This is why the oxygen spectrum in the air appears rather well resolved even for pressures of the order of half an atmosphere [Artman, 1953 and 1954]. It appears probable that the study of ammonia, where the fine structure appears around $100 \mathrm{~mm} \mathrm{Hg}$, could give qualitative information on the behavior of oxygen at high pressure [Maryott and Birnbaum, 1955].

The study of chloroform gives us an example of the complexity of the absorption and dispersion problem when we have to take into account the blending of closely neighboring lines. Since here absorption is studied in connection with dispersion, the results underline the fact that a notable dispersion can only be observed if there is a high degree of absorption. This result is used in the study of the atmosphere [Kerr, 1951].

B. The spectra of ammonia and chloroform-The ammonia molecule $\left(\mathrm{NH}_{3}\right)$ is symmetric and has the shape of a regular pyramid whose apex is occupied by a nitrogen atom (see fig. 22a). Although the lines of the rotational and vibrational spectra of this molecule are in the infra red [Herzberg, 1951], there exists absorption in the hertzian domain. It is caused by a phenomenon of "inversion" which corresponds to the "passage" of the nitrogen atom from one side of the plane $\mathrm{HHH}$ to the other. The vibrational energy of the nitrogen atom "on the right" of this plane is slightly different from the vibrational level "on the left" so that the vibrational level is split. The transition between those two states, which is made possible by a "tunnel effect" and known as "inversion," is responsible for the observed absorption of the molecule in the ground state (around 24 $\mathrm{Gc} / \mathrm{s}$ ) (fig. 22b).

The inversion spectrum of ammonia belongs to the more general class of spectra caused by internal motion of a molecule. One will find an extensive discussion of this phenomenon in chapter 12 of the book by Townes and Schalow [1955]. Consequently, we limit ourselves to indicating the aspects of this phenomenon which are of particular interest to us.

The inversion spectrum of ammonia presents a fine structure. This fine structure is caused by the interaction between the inversion and the rotation of the molecule. A classic but qualitative explanation is to suppose that rotation modifies the energy level of vibration by means of centrifugal forces Computations give:

$$
\nu_{J K}=\nu_{0}-a\left[J(J+1)-K^{2}\right]+b K^{2}+\ldots,
$$

$J$ and $K$ are the quantic numbers of the rotational levels, $\nu_{0}=23787 \mathrm{Mc} / \mathrm{s}$ and $a$ and $b$ are the fine structure constants for which experience gives $a=151.5 \mathrm{Mc} / \mathrm{s}$ and $b=59.9 \mathrm{Mc} / \mathrm{s}$.

When the gas pressure becomes larger than 50 to $100 \mathrm{~mm} \mathrm{Hg}$, the fine structure disappears and the spectrum is then a unique line. But the "resonance frequency" $\nu_{0}$ which corresponds to this line is not pressure independent any more. It decreases with increasing pressure and for pressures close to or greater than 2 atm the frequency $\nu_{0}$ must be considered as 0 . The absorption loses its resonant character. This phenomenon has been observed experimentally [Bleaney and Loubser, 1948; Smith, 1948; Weingarten, 1948] and theoretical explanations have been given [Anderson, 1949b; Margenau, 1949a and 1949b].

Another phenomenon becomes important for relatively high pressures. The width parameter $\Delta \nu$ of the line ceases to be proportional to the gas pressure, it increases more slowly as the pressure nears $1 \mathrm{~atm}$. It is completely stable above $4 \mathrm{~atm}$ [Birnbaum and Maryott, 1953a and 1953b; Bleaney and Loubser, 1948 and 1950; Weingarten, 1948]. This effect is attributed to multiple collisions (more than two molecules collide at the same time) [Bleaney and Loubser, 1948 and 1950], while more complicated explanations have been proposed by the several theories of shock broadening of lines.

The ammonia molecule has been the object of numerous experimental and theoretical works. Although it is the best known molecule from a microwave spectroscopy viewpoint, the sustained interest in its study continues to bring forth new works even today.

The chloroform molecule is not as well known in the hyperfrequency range. It is also a symmetric molecule but its rotational spectrum is located squarely in the centimetric wave region. The structure of the molecule (fig. 23) and its rotational constant $B$ have been determined by the techniques of microwave spectroscopy [Ghosh, Trambarulo, and Gordy, 1952; Smith and Unterberger, 1950; Unterberger, Trambarulo, and Smith, 1950]. The rotational spectrum is made up of lines whose frequency are given by the general relation [Townes and Schalow, 1955]:

$$
\nu_{J}=2 B(J+1), \quad(J=0,1,2 \ldots),
$$

for chloroform

$$
B=3302 \mathrm{Mc} / \mathrm{s} \text {. }
$$

Spectroscopic measurements are usually made under low pressure so as to maintain high resolution. This type of measurement has yielded information concerning the position of the lines of different substances, on the fine and hyperfine structure, 
a

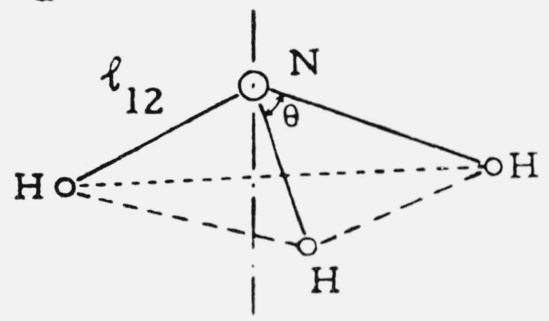

$$
\begin{aligned}
h_{12} & =1,014 \cdot 10^{-10} \mathrm{~m} \\
& =106^{\circ} 47^{\prime}
\end{aligned}
$$

$\mathrm{b}$

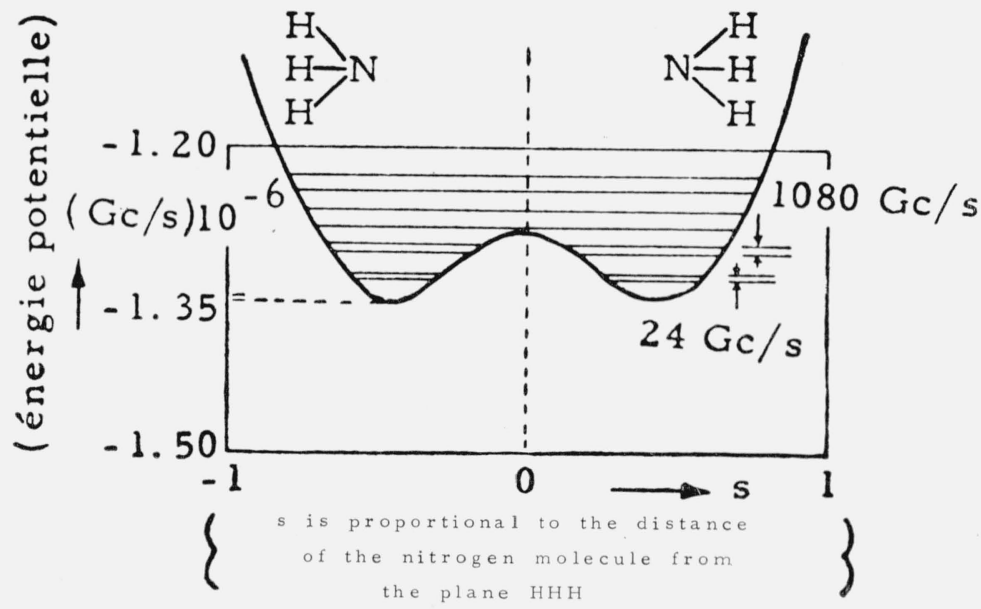

Figure 22. Ammonia molecule.

a. Lire $1-106^{\circ} 47^{\prime}$. Molecular structure of ammomia [Herzberg, 1951; Weiss and Strandberg, 1951]. b. Its inversion mechanism [Townes and Schalow, 1955].

CHLOROFORME

Structure de la molécule du chloroforme

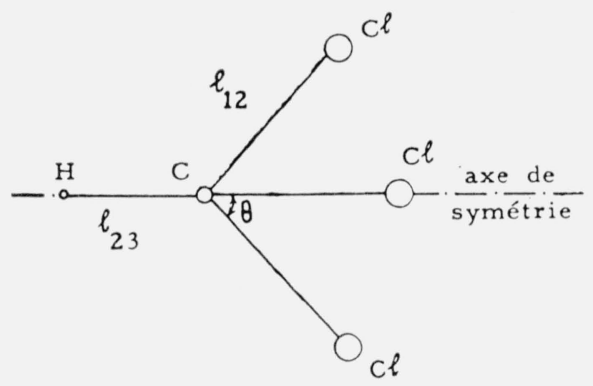

$$
\begin{aligned}
\ell_{12} & =1,767 \cdot 10^{-10} \mathrm{~m} \\
\ell_{23} & =1,073 \cdot 10^{-10} \mathrm{~m} \\
\theta & =110^{\circ} 24^{\prime} \\
B & =3302 \mathrm{Mc} / \mathrm{s}
\end{aligned}
$$

Figure 23. Chloroform molecule [Gosh et al. 1952; Unterberger et al, 1950].

etc. . . [Townes and Schalow, 1955]. Nevertheless, with a few exceptions (ammonia, OCS ...), we lack data on the absolute intensity of the absorption and on the shapes of the lines at high pressure. Our instrument is well-adjusted to this type of study and we have used it to study chloroform.

C. Study of the absorption at constant temperatures1. Absorption measurements for ammonia have been made in the band from 7 to $12 \mathrm{Gc} / \mathrm{s}$ (klystron X $13 \mathrm{~B}$ ). For each selected frequency in this band, we obtain a curve of the absorption $(\alpha)$ versus the pressure $(p)$ by decreasing the pressure from about $300 \mathrm{~mm} \mathrm{Hg}$ to $\mathrm{O}$. The temperature of the cavity is maintained at $0{ }^{\circ} \mathrm{C}$ (except for absorption versus temperature measurement). The absolute calibration of the instrument (sec. 1.9) is used during these measurements (for instance, $v=900 \mathrm{Mc} / \mathrm{s}^{2}$ and $k=0.70$ which correspond to a sensitivity of $1.346 \times 10^{-6}$ neper $/ \mathrm{m} /$ $\mu \mathrm{sec})$.
Three curves $(\alpha, p)$ are shown on figure 24 for frequencies 7.30, 9.33, and $11.40 \mathrm{Gc} / \mathrm{s}$. The parabolic shape of these curves (this was expected since the used frequencies are located on the lower wing of the ammonia line, see sec. B and 2.3) is well-marked. This feature appears even better on figure 24 where the same curves are drawn on a logarithmic scale. One notes that the absorption is of the form $\alpha=\left(C^{t e}\right) \times p^{\beta}$, the slope $\beta$ is equal to 1.96 for $50<p<150 \mathrm{~mm} \mathrm{Hg}$. The slope tends toward 2 for $p<50 \mathrm{~mm} \mathrm{Hg}$ and decreases progressively above $150 \mathrm{~mm} \mathrm{Hg}$. The absorption is thus given, as a function of pressure by a quadratic parabola as predicted by the theory (sec. 2.3). The constant proportionality is such that we have

$$
\begin{gathered}
\alpha=5.0 \cdot 10^{-3} \text { neper } / \mathrm{m} \text { to } 9.33 \mathrm{Gc} / \mathrm{s} \\
100 \mathrm{~mm} \mathrm{Hg} \text { and } 0{ }^{\circ} \mathrm{C} .
\end{gathered}
$$




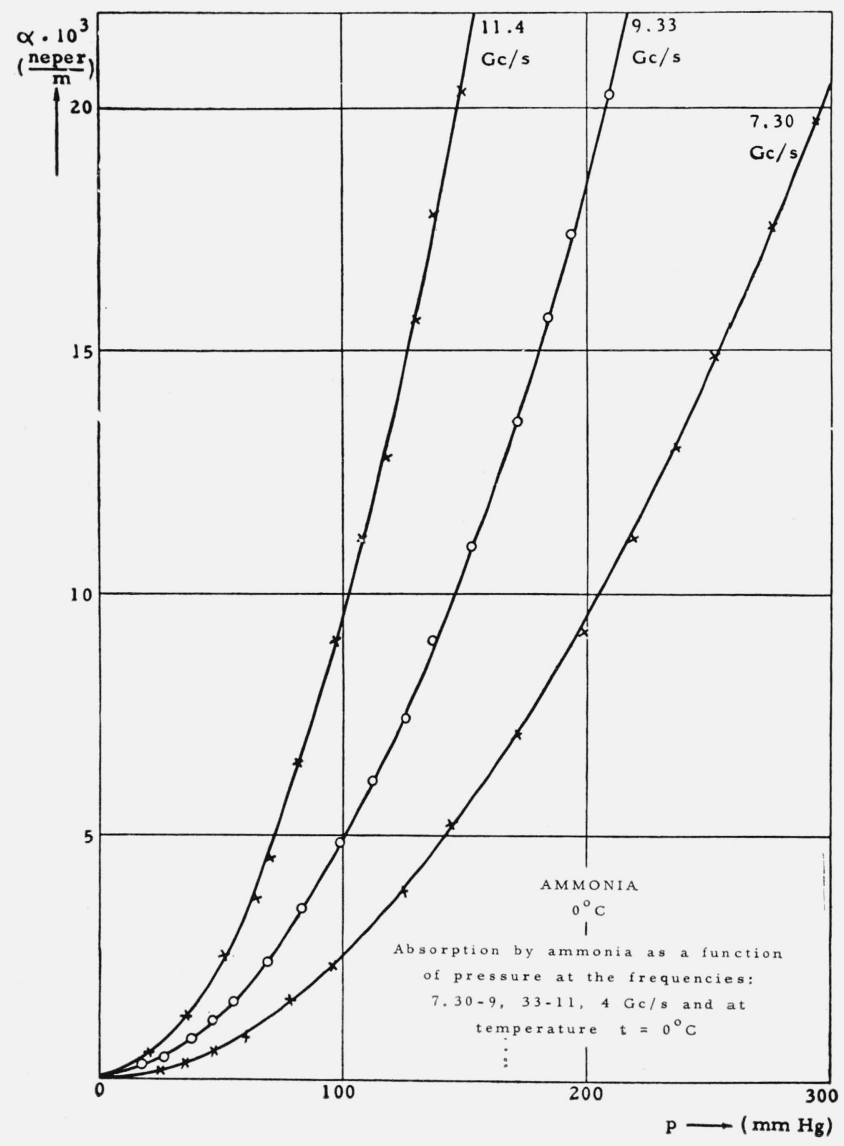

Figure 24. Ammonia absorption (linear scale).

The absorption is expressed in accordance with the theory of Van Vleck-Weisskopf by (2.33). When the gas pressure is sufficiently high (above 50 $\mathrm{mm} \mathrm{Hg}$ ) the fine structure of ammonia is masked (see sec. 3.2B) because the width of the lines becomes large with respect to the separation between the different resonant frequencies $\nu_{j k}$.

This fact can be expressed analytically if one is willing to lump all the $\nu_{j k}$ frequencies into one "resonant" frequency $\nu_{0}$, although the frequencies $\nu_{j k}$ occupy a rather large band "from 20 to $30 \mathrm{Gc} / \mathrm{s}$." Since the band occupied by the principal lines is narrower (from 21 to $26 \mathrm{Gc} / \mathrm{s}$ ) while the line width parameter becomes of the order of $10 \mathrm{Gc} / \mathrm{s}$ at half an atmosphere of pressure, the approximation $\nu_{j k} \approx \nu_{0}$ seems legitimate. It becomes even more so as one moves further away from the resonant frequency $\nu_{0}$ toward the wing of line. (2.33) then takes the form

$$
\begin{aligned}
& \alpha=g \cdot 10^{-5}\left(\frac{273}{T}\right)^{2.5} \cdot \nu^{2} \cdot\left[\frac{\Delta \nu}{\left(\nu_{0}-\nu\right)^{2}+(\Delta \nu)^{2}}\right. \\
&\left.+\frac{\Delta \nu}{\left(\nu_{0}+\nu\right)^{2}+(\Delta \nu)^{2}}\right] \cdot p,(\mathrm{np} / \mathrm{m}) .
\end{aligned}
$$

In this relation $g$ is a constant factor (of the order of 3 as we shall see later), $p$ is measured in $\mathrm{mm} \mathrm{Hg}$, $\nu$ and $\nu_{0}$ in $\mathrm{Gc} / \mathrm{s}, \Delta \nu$ keeps its usual meaning of width parameter, while the factor $(273 / T)^{2.5}$ (equal to 1 for $t=0^{\circ} \mathrm{C}$ ) takes into account the influence of the temperature.

Such a representation of absorption by a single line has been suggested for different reasons and under different conditions by several authors [Birnbaum, 1949 and 1950; Birnbaum and Maryott, 1953a and 1953b; Bleaney and Loubser, 1948 and 1950; Walter and Hershberger, 1946; Weingarten, 1948]. Our viewpoint is the following: we will try to apply (3.17) to the totality of known results concerning the absorption of ammonia at hyperfrequency energies (bands from 2 to $45 \mathrm{Gc} / \mathrm{s}$ ). These results have been given by many workers and we are going to pay special attention to the pressure domain which corresponds to the operating range of our instrument $(10 \mathrm{~mm} \mathrm{Hg}$ to $1 \mathrm{~atm})$. Our aim is twofold:

$\alpha$. Check, under the condition mentioned above, on the validity of the VV-W formula. Studies of this type have been undertaken by other workers but they had different goals and used different means. They would limit themselves to a region around resonance and would make direct use of the spectroscopic data of ammonia [Bleaney and Penrose, 
1947b]. Or they would verify the law $\alpha=\left(C^{t e}\right) p^{2}$ for average pressure and far from resonance [Battaglia, Bruin, and Gozzini, 1958;Birnbaum, Kryder, and Lyons, 1951; Birnbaum and Maryott, 1953a and 1953b; Walter and Hershberger, 1946]. Or they would work at very high pressures up to 6 or $7 \mathrm{~atm}$ [Bleaney and Loubser, 1948 and 1950; Weingarten, 1948]. Although our measurements are limited in frequencies and pressure, we consider them as rather precise and by comparing them against all other results agree together and to what extent they can be interpreted by the $\mathrm{VV}-\mathrm{W}$ formula.

$\beta$. Besides this theoretical interest, a formula such as (3.17), brought into the best possible agreement with experimental results, would furnish us, in first approximation, a valuable indication of the relative calibration of our instrument. The practical interest would be great mainly when one changes frequency or temperature.

The agreement between the different experimental results is at first glance rather satisfactory, although there appears considerable discrepancies here and there. We shall not dwell on the detailed comparison which should take into account the date of each measurement, and the experimental technique used. Such a comparison can be better made by taking a careful look at figures 25 to 27 and at table 3 .

We proceed next to the adjustment of (3.17) with these results. Such an adjustment must respect the fact that the integrated intensity of a line (see sec. 2.3A):

$$
I_{\mathrm{int}}=\int_{0}^{\infty} \frac{\alpha}{\nu^{2}} d \nu
$$

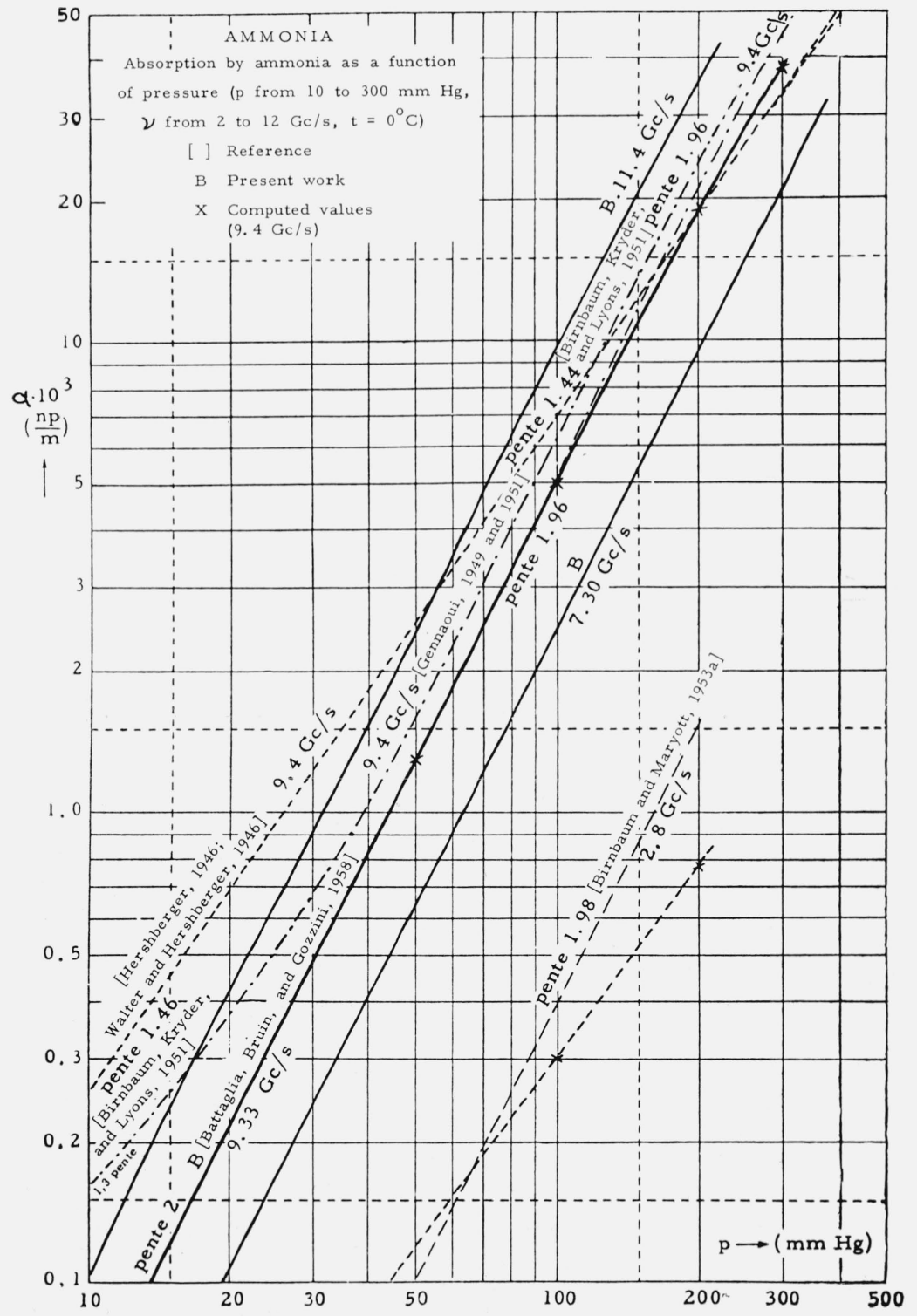

Figure 25. Ammonia absorption (logarithmic scales). 


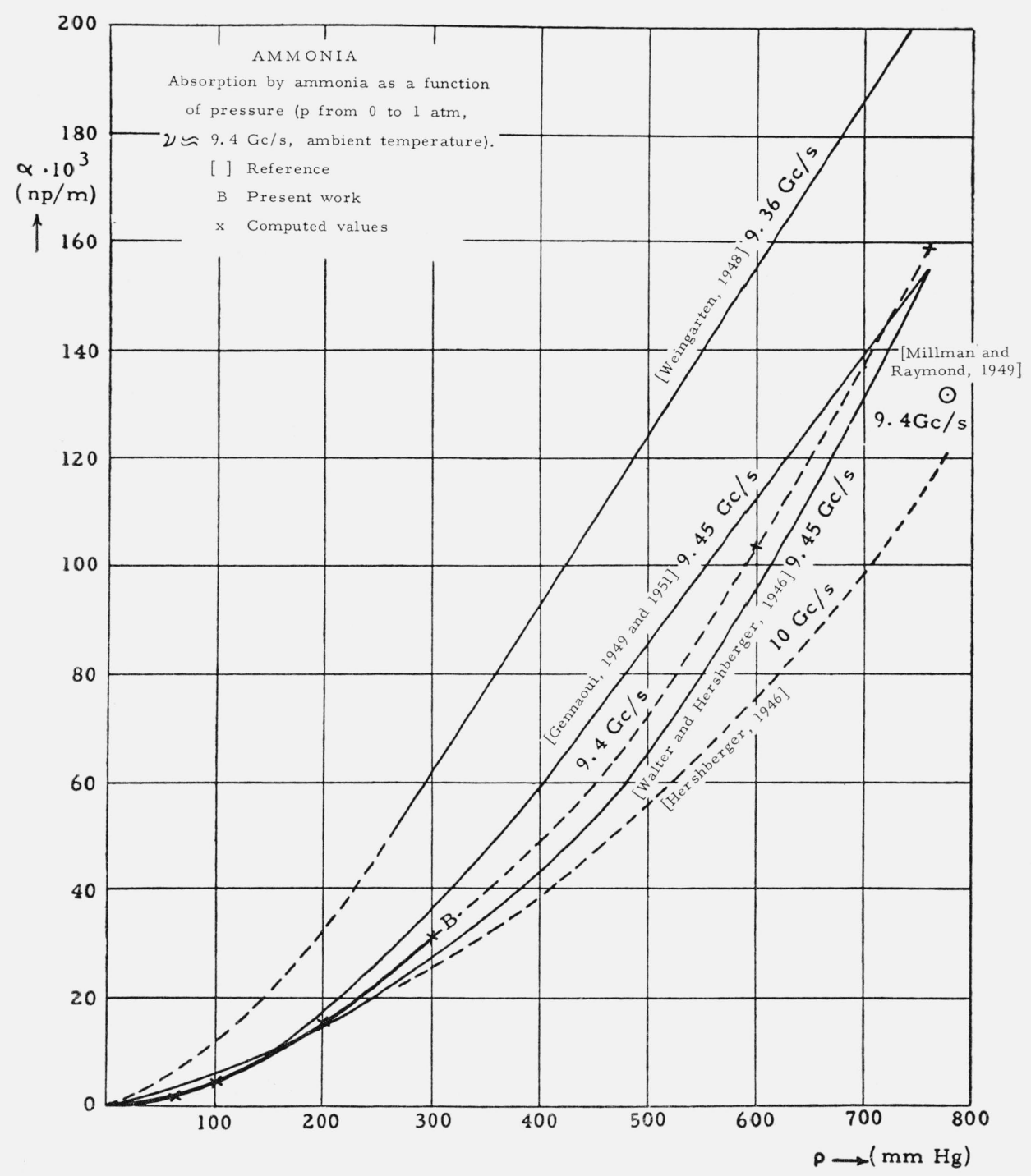

FIGURE 26. Ammonia absorption (up to a pressure of 1 atmosphere).

must be independent of the presumed line shape. Weingarten [1948] whose computation gives $I_{\text {int }} / p$ $=9 \times 10^{-5} \quad($ neper $/ \mathrm{m}) /(\mathrm{Gc} / \mathrm{s}) / \mathrm{mm} \mathrm{Hg}$, has shown that this condition is sufficiently well verified by experiences. When one tries to adjust (3.17), the values selected for $\nu_{0}$ and $\Delta \nu$ can be more or less arbitrary. 'One knows that in fact these quantities are, in the case of ammonia, complicated functions of the gas pressure (see sec. 3.2B).

In order to obtain the best possible agreement with experiments we have decided that the factor $g$ was a free parameter. This does not have any theoretical justification but we have so decided on account of our second objective. Anticipating the results of this adjustment, we note (fig. 28) that $g$ doesn't depart very much from an average value of 3.5 (the small value of $g$ at $p=50 \mathrm{~mm} \mathrm{H}$. could be caused by the fact that at this pressure the fine structure remains partially resolved - see fig.
27). Birnbaum and Maryott [1954] are led to adopt a constant value for this factor which is, after conversion of the units, somewhere in between 3.8 and 4.2 according to the results one selects, while a theoretical computation yields $g$ of the order of 3.6 (2.13).

We thus have three parameters that we can adjust: $\nu_{0}, \Delta \nu$, and $g$. We have fixed their value by requiring that (3.17) give, for each selected pressure $(50,100,300,600$, and $760 \mathrm{~mm} \mathrm{Hg})$, the most probable measured values corresponding to three points on each line. These three points are: one at $\nu=9.4 \mathrm{Gc} / \mathrm{s}$ and giving preference to our own measurements; the other is selected toward the top of the line; and the third one selected at an intermediate position on the lower wing of the line. We thus have required that the agreement be best on the left of the resonance frequency.

One then obtains for each line, and for each pres- 
TABLE 3

\begin{tabular}{|c|c|c|c|c|c|c|}
\hline Reference & Freq. & Pressure & $\begin{array}{l}\text { Temper- } \\
\text { ature }\end{array}$ & Technique & Precision, sensitivity & Remarks \\
\hline Cleeton-Williams [1934] & $\begin{array}{l}G c / s \\
\quad 7.9 \\
\text { to } \\
28.3\end{array}$ & $1 \mathrm{~atm}$ & ambient & $\begin{array}{l}\text { Mirror spectrometer. } \\
\text { magnetrons }\end{array}$ & $\begin{array}{c}\text { neper } / m \\
\text { The error on } \lambda \text { less than } 1 \% \text {-- }\end{array}$ & $\begin{array}{l}\text { For the infrared, the Dennison interpre- } \\
\text { tation formula (1928) was used. }\end{array}$ \\
\hline Hershberger [1946] ....... & $\begin{array}{c}24 \\
\text { and } \\
10\end{array}$ & $\begin{array}{r}1 \text { atm \& } \\
0 \text { to } \\
1 \text { atm }\end{array}$ & ambient & Waveguide $(30 \mathrm{ft}) \ldots \ldots$ & Sensitivity of 0.005 neper $/ \mathrm{m}_{-}$ & Other gases were studied. \\
\hline $\begin{array}{l}\text { Walter-Hershberger } \\
\text { [1946] }\end{array}$ & $\begin{array}{l}24.2 \\
\text { and } \\
9.45\end{array}$ & $\begin{array}{r}0 \text { to } \\
1 \mathrm{~atm}\end{array}$ & ambient & Waveguide+absorber....... & $\begin{array}{l}\text { Sensitivity } 0.002 \text { Precision } \\
\quad \pm 5 \%\end{array}$ & $\begin{array}{l}\text { Formulas were analogous to the quoted } \\
\text { equation (3.17). }\end{array}$ \\
\hline Bleaney-Penrose [1947]_- & $\begin{array}{l}18.9 \\
\text { to } \\
27.6\end{array}$ & $\begin{array}{r}0 \text { to } \\
600 \\
\mathrm{~mm} \mathrm{Hg}\end{array}$ & ambient & $\begin{array}{l}\text { Guide } 1 \mathrm{~m} 3 \text { klystrons, } \\
\text { bolometer }\end{array}$ & Precision $\pm 4 \%$ at $600 \mathrm{~mm} \mathrm{Hg}_{-}$ & $\begin{array}{l}\text { Formula for } \nu_{J K} \text { and }(\Delta \nu)_{J K} \text {. Agreement } \\
\text { with computation at } 100 \mathrm{~mm} \mathrm{Hg} \text {. } \\
\text { Mediocre agreement at } 600 \mathrm{~mm} \\
\text { Insufficient frequency band. }\end{array}$ \\
\hline Weingarten [1948] ...... & $\begin{array}{l}9.36 \\
\text { to } \\
34.5\end{array}$ & $\begin{array}{r}0 \text { to } \\
7 \mathrm{~atm}\end{array}$ & $25^{\circ} \mathrm{C}$ & $\begin{array}{l}\text { Nonresonant cavity, bolom- } \\
\text { eter, magnetrons }\end{array}$ & Precision \pm 3 to $5 \% \ldots$ & $\begin{array}{l}\text { "Unified line" in good agreement at } \\
p>1 \mathrm{~atm} \text {. Large error possible around } \\
9.36 \mathrm{Gc} / \mathrm{s} \text { (small cavity). }\end{array}$ \\
\hline Bleaney-Loubser [1950]_- & $\begin{array}{l}3.7 \\
\text { to } \\
37\end{array}$ & $\begin{array}{r}0 \text { to } \\
6 \mathrm{~atm}\end{array}$ & ambient & $\begin{array}{l}\text { Resonant cavities, crystal. } \\
Q \text { measurement }\end{array}$ & Precision around \pm 2 to $3 \%$-- & $\begin{array}{l}\text { (3.17) adjusted at } p q q . \text { atm (departure } \\
\text { at } 1 \mathrm{~atm} \text { ). Insufficient measurement } \\
\text { at low pressure. }\end{array}$ \\
\hline Gennaoui [1951] ......... & 9.45 & $\begin{array}{r}0 \text { to } \\
1 \mathrm{~atm}\end{array}$ & ambient & $\begin{array}{l}\text { Resonant cavities, Q meas- } \\
\text { urement }\end{array}$ & Sensitivity 0.002 neper $/ \mathrm{m}_{\text {..... }}$ & Verification of the law $\left.\alpha=(C t e) \cdot p^{2}.\right)$ \\
\hline $\begin{array}{l}\text { Birnbaum-Kryder- } \\
\text { Lyons [1951] }\end{array}$ & 9.28 & $\begin{array}{r}0 \text { to } \\
200 \\
\mathrm{mmg}\end{array}$ & $\begin{array}{r}24.7 \stackrel{\&}{2} .5^{\circ} \mathrm{C} \\
\end{array}$ & $\begin{array}{l}\text { Two resonant cavities, } \\
\text { swept klystron, labeling }\end{array}$ & $\begin{array}{l}\text { Percision } \pm 2 \%, \text { Sensitivity } \\
\quad 10^{-4} \text { neper } / \mathrm{m}\end{array}$ & Verification of the law $\alpha=(C t e) \cdot p^{2}$ \\
\hline $\begin{array}{l}\text { Birnbaum-Maryott } \\
{[1953]}\end{array}$ & $\begin{array}{r}2.8 \\
(1.8 \\
\text { to } \\
2.8)\end{array}$ & $\begin{array}{r}100 \\
(300) \\
\mathrm{mm} H\end{array}$ & $\frac{-12 \text { to }}{100^{\circ} \mathrm{C}}$ & $\begin{array}{l}\text { Same as above plus a few } \\
\text { improvements }\end{array}$ & Precision around \pm 2 to $3 \%$-- & $\begin{array}{l}\text { Check of the VV-W formula. Verifica- } \\
\text { tion of the law } \alpha=(C t e) \cdot p^{2} \text {. Dis- } \\
\text { cussion. }\end{array}$ \\
\hline $\begin{array}{l}\text { Battaglia-Bruin-Gozzini } \\
\text { [1958] }\end{array}$ & 9.4 & $\begin{array}{r}0 \text { to } \\
250 \\
\mathrm{~mm} \mathrm{Hg}\end{array}$ & $\begin{array}{r}0 \text { to } \\
15^{\circ} \mathrm{C}\end{array}$ & Same as ours.................. & $\begin{array}{l}\text { Same order of magnitude as } \\
\text { ours }\end{array}$ & Verification of the law $\alpha=(C t e) \cdot p^{2}$. \\
\hline Present work_........ & $\begin{array}{l}7 \\
\text { to } \\
12\end{array}$ & $\begin{array}{r}0 \text { to } \\
250 \\
\mathrm{~mm} \mathrm{Hg}\end{array}$ & $50^{\circ} \stackrel{\text { to }}{\mathrm{C}}$ & $\begin{array}{l}\text { Two resonant cavities, } \\
\text { pulses }\end{array}$ & $\begin{array}{l}\text { Precision } \pm 1 \text { to } 2 \% \text {, Sensi- } \\
\quad \text { tivity } 10^{-5} \text { neper } / \mathrm{m}\end{array}$ & $\begin{array}{l}\text { Verification of the law } \alpha=(C t e) \cdot p^{2} \text {. } \\
\text { Check on the } V \mathrm{~V}-\mathrm{W} \text { formula. }\end{array}$ \\
\hline
\end{tabular}

sure a system of three equations in three unknowns. Since we did not want to approximate (3.17), these systems are complicated. They have been solved graphically. The results (valid at $0{ }^{\circ} \mathrm{C}$ ) are indicated in table 4 and on figure 28 where we have plotted for comparison the values proposed by other workers. One notices again the well-known effect (see sec. $3.2 \mathrm{~B}$ ) of the decrease of $\nu_{0}$ and $\Delta \nu / p$ with pressure. As a consequence $\nu_{0}$ and $\Delta \nu$ become practically equal $\left(\nu_{0} \approx \Delta \nu \approx 15 \mathrm{Gc} / \mathrm{s}\right)$ around $1 \mathrm{~atm}$.

As a first approximation $\Delta \nu$ can be expressed by the relation:

$\Delta \nu=(30,4-0,014 p) \cdot p \cdot 10^{-3}$,

$$
(\mathrm{Gc} / \mathrm{s} \text {, with } p \text { in } \mathrm{mm} \mathrm{Hg} \text { ). }
$$

A similar relation $\left(\Delta \nu=(24.5-0.008 p) \times p \times 10^{-3}\right.$ for ambient temperature) has been suggested by Bleaney and Loubser [1948 and 1950] who have justified this expression by appealing to theoretical considerations taking into account the effect of multiple collisions.

TABLE 4

\begin{tabular}{|c|c|c|c|c|c|}
\hline \multirow[b]{2}{*}{$\begin{array}{l}\nu_{3}(\mathrm{Gc} / \mathrm{s}) \\
\Delta \nu(\mathrm{Gc} / \mathrm{s})\end{array}$} & \multicolumn{5}{|c|}{ Pressure $(\mathrm{mm} \mathrm{Hg})$} \\
\hline & $\begin{array}{l}50 \\
23.0 \\
1.75 \\
2.86\end{array}$ & $\begin{array}{c}100 \\
22.9 \\
2.9 \\
3.22\end{array}$ & $\begin{array}{c}300 \\
22.0 \\
7.8 \\
3.30\end{array}$ & $\begin{array}{c}600 \\
81.3 \\
13.2 \\
3.66\end{array}$ & $\begin{array}{c}760 \\
15.5 \\
14.4 \\
3.71\end{array}$ \\
\hline
\end{tabular}

Once the parameters $\nu_{0}$, and $\Delta \nu$, and $g$ have been determined, we have recalculated by means of (3.17) the lines of figure 27 and the absorption curves $\alpha(p)$ of figures 25 and 26 . We make the following conclusions: $\alpha$. If one tries to obtain the best possible agreement between the computation and the measurements on the left of the resonance, the discrepancy on the other side of the line becomes rather large. Birnbaum and Maryott [1953a] have already shown that when a line computed according to the VV-W formula is in good agreement around resonance, the absorption at 2.8 and $9 \mathrm{Gc} / \mathrm{s}$ is larger by 40 percent. Bleaney and Loubser [1948 and 1950] using methods similar to ours have been able to bring good agreement between measured and computed line but their best success occurs at high pressure, while they still have appreciable discrepancy in the neighborhood of $1 \mathrm{~atm}$. It thus appears that, for the condition under which we work $(10<p<760 \mathrm{~mm} \mathrm{Hg})$ a single line, of the VV-W type could not be adopted to measurement over the whole spectrum (from a few Gc/s to a few tens $\mathrm{Gc} / \mathrm{s}$ ) with the precision of the order of the experimental error (see sec. 2.3D). The molecular interaction appears to become too complicated in this domain and one begins to wonder if the statistical theory would not give better results (see the work of D. F. Smith [Mazur and Mandel, 1956a and 1956b] who had in mind pressures of a few atm). Bearing in mind these reservations as well as a certain ambiguity in the measurement one should not exclude the 


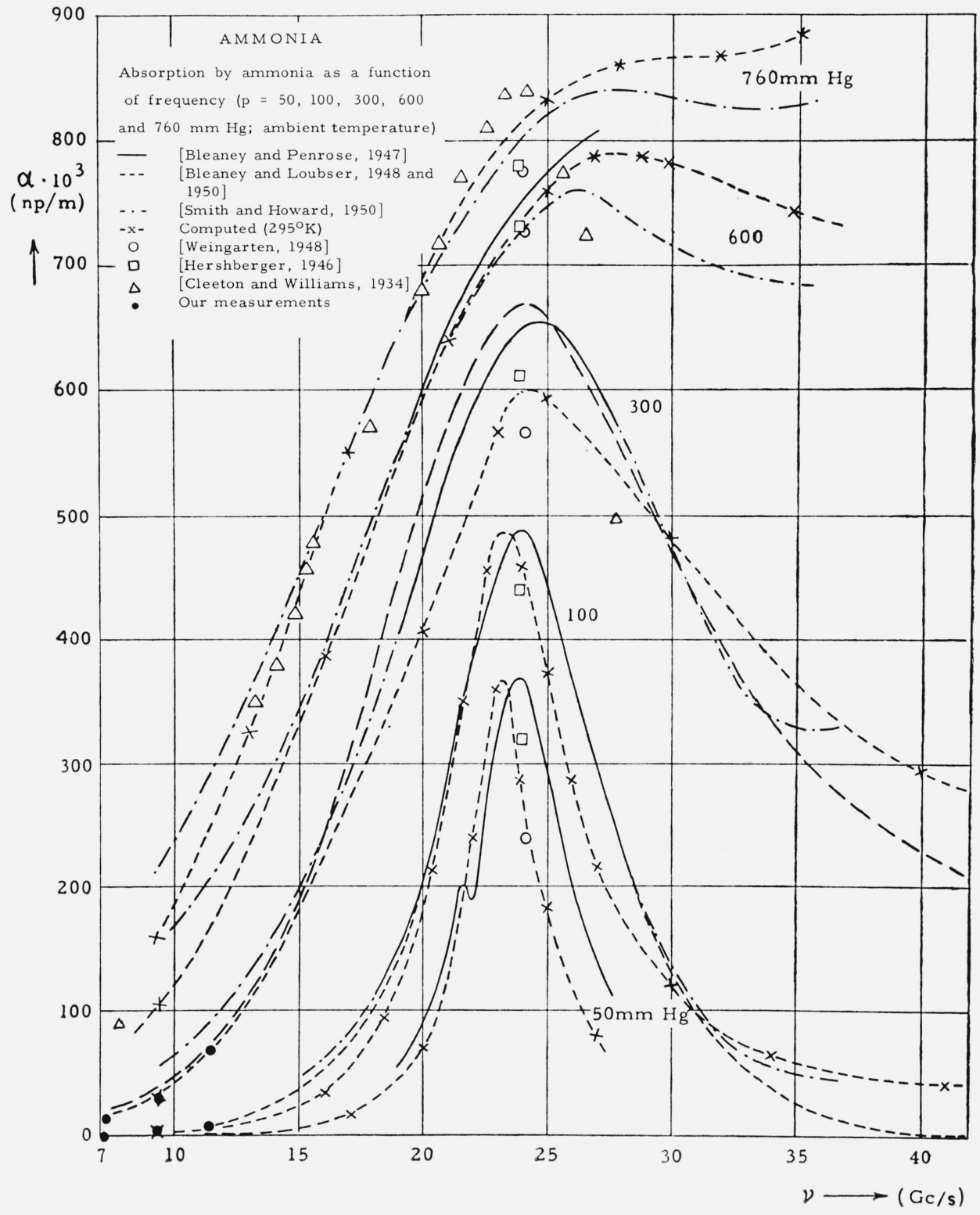

FiguRE 27. Ammonia absorption spectrum for hyperfrequencies (average pressure).

representation of absorption by a "single line" of the VV-W type as a first approximation. The asymmetric character of the lines as well as the shift to the right of the absorption maximum are evidenced in figure 27. Such a representation is of some use if one does not try to give it a rigorous significance and if one does not try to overestimate its meaning.

$\beta$. Let us now consider the $\alpha(p)$ curves giving the absorption as a function of pressure. When one uses (3.17) under the particular conditions $p=100 \mathrm{~mm} \mathrm{Hg}$ and $t=0{ }^{\circ} \mathrm{C}$ one obtains:

$$
\alpha=9.338 \cdot \nu^{2}\left[\frac{1}{(22.9-\nu)^{2}+8.41}+\frac{1}{(22.9+\nu)^{2}+8.41}\right]
$$

$\cdot 10^{-3},(\mathrm{np} / \mathrm{m})$, at $p=100 \mathrm{~mm} \mathrm{Hg}$ and $t=0^{\circ} \mathrm{C}, \nu$ in $\mathrm{Gc} / \mathrm{s}$ (this expression must be multiplied by the factor $(273 / T)^{2.5}$ for temperatures $\left.t \neq 0{ }^{\circ} \mathrm{C}\right)$, and this relation is useful for the relative calibration of our instrument.

If one operates sufficiently far from resonance and if the pressure is not too high, (3.17) can be written as (see sec. 2.3C):

$$
\alpha=2 g \cdot 10^{-5}\left(\frac{273}{T}\right)^{2.5} \cdot \frac{1+\left(\frac{\nu}{\nu_{0}}\right)^{2}}{\left(\frac{\nu_{0}}{\nu}-\frac{\nu}{\nu_{0}}\right)^{2}} \cdot(\Delta \nu) p .
$$

The error (in excess) caused by the use of (3.21) instead of (3.17) can be estimated with the help of table 5 ( $\nu_{0}$ and $\Delta \nu$ have the values indicated on table 4 ). 


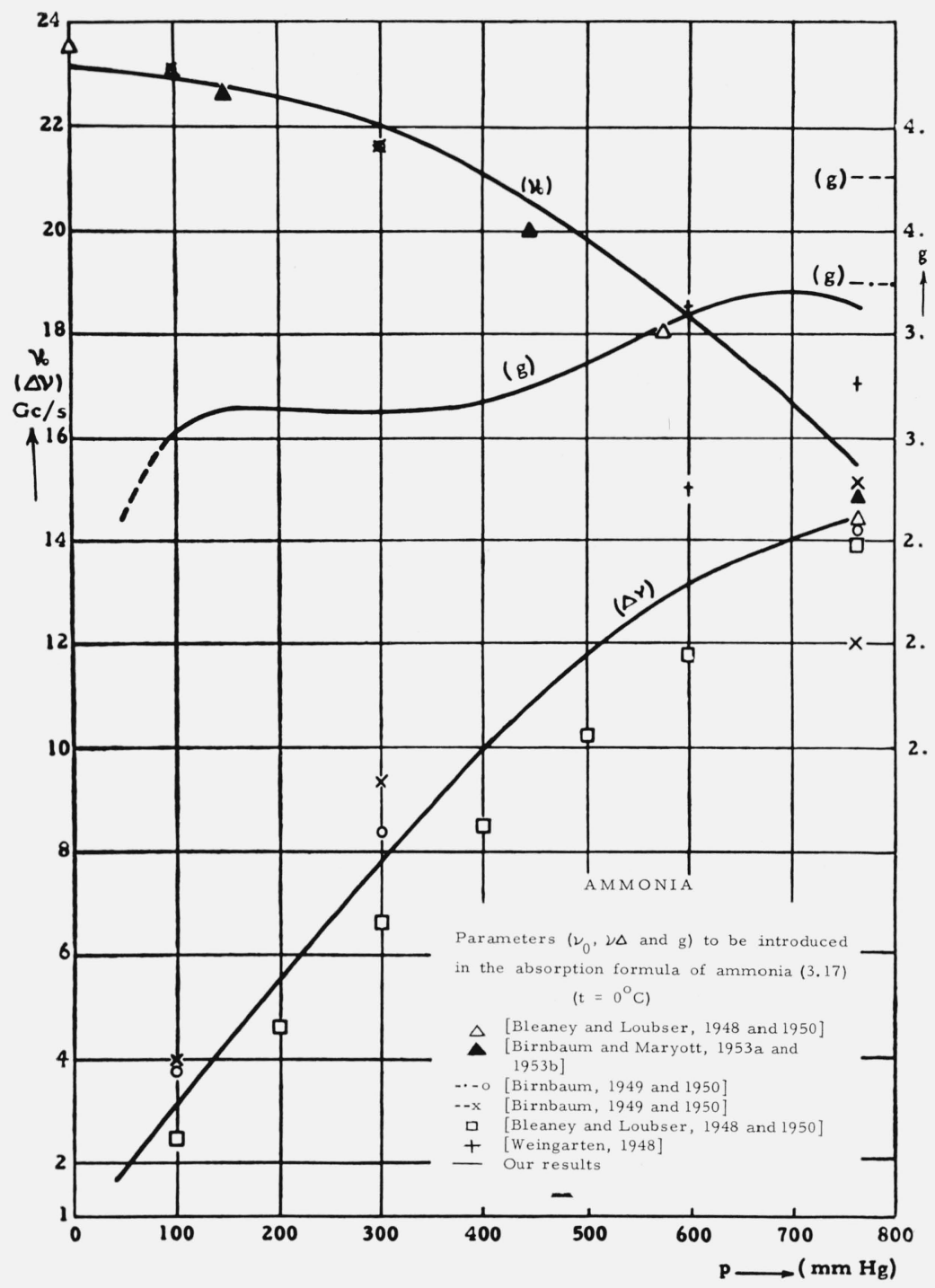

Figure 28. Absorption parameters for ammonia (eq. 3.17).

TABLE 5

\begin{tabular}{l|c|c|c}
\hline \hline \multicolumn{2}{c|}{$p=100 \mathrm{~mm} \mathrm{Hg}$} & \multicolumn{2}{c}{$300 \mathrm{~mm} \mathrm{Hg}$} \\
\hline $\begin{array}{l}\nu=2.8 \mathrm{Gc} / \mathrm{s} \\
\text { Error }+1.3 \%\end{array}$ & $\begin{array}{c}9.4 \mathrm{Gc} / \mathrm{s} \\
+3.5 \%\end{array}$ & $\begin{array}{c}2.8 \mathrm{Gc} / \mathrm{s} \\
+13.7 \%\end{array}$ & $\begin{array}{c}9.4 \mathrm{Gc} / \mathrm{s} \\
+31 \%\end{array}$ \\
\hline
\end{tabular}

The error is of a few percent or more and increases quickly with the pressure as one approaches the resonance frequency $\nu_{0}$. In all cases where (3.21) is judged valid, and if one supposes that in the domain of its application $\nu_{0}$ does not change appreciably while $\Delta \nu$ remains proportional to the pressure, one may write the simple formula:

$$
\alpha=(C t e) p^{2} \text { (at constant temperature). }
$$

Experiments verify this conclusion (fig. 24). Our measurements give:

$$
\begin{aligned}
& \alpha=5 \cdot 10^{-7} \cdot p^{2},(n p / m), \\
& \quad\left(p<150 \mathrm{~mm} \mathrm{Hg},{ }_{t}=0{ }^{\circ} \mathrm{C}, \nu=9.33 \mathrm{Gc} / \mathrm{s}\right) .
\end{aligned}
$$

This result is equivalent to admitting that $\Delta \nu / p=0.029 \mathrm{Gc} / \mathrm{s} / \mathrm{mm} \mathrm{Hg}$. Using spectroscopic data of ammonia, Bleaney and Loubser [1948 and 1950] have computed [Bleaney and Penrose, 1947b] an average value of $\Delta \nu$ with the formula:

$$
\Delta \nu=\frac{\sum_{J K} I_{J K} \Delta \nu_{J K}}{\sum_{J K} I_{J K}} .
$$


$I_{j k}$ is the integrated intensity of the fine line $(J, K)$ and they find that $\Delta \nu / p=0.0245 \mathrm{Gc} / \mathrm{s} / \mathrm{mm} \mathrm{Hg}$. The corresponding absorption is approximately 15 percent lower than the measured value. Another computation of Birnbaum and Maryott [1953a] brings the discrepancy to about 40 percent. As mentioned above these remarks reinforce the one made previously concerning the quantitative validity of the $\mathrm{VV}-\dot{W}$ formula over the totality of the spectrum band of ammonia.

2. The absorption problem presents itself in a different manner in the case of chlorotorm. The frequency band used is located between the first two lines of the rotation spectrum, at 6.6 and $13.2 \mathrm{Gc} / \mathrm{s}$, respectively (see sec. $3.2 \mathrm{~B}$ ). We will have to consider not only the cumulative effect of those first two lines but also the influence of the residual absorption caused by all the other lines located higher up.

Since the saturation pressure of chloroform vapor is limited to $61 \mathrm{~mm} \mathrm{Hg}$ at $0{ }^{\circ} \mathrm{C}$, we have worked at $t=30{ }^{\circ} \mathrm{C}$ which allows us to go to a pressure of about
$150 \mathrm{~mm} \mathrm{Hg}$. The precision in the measurements is affected by the weakness of the absorption at pressures smaller than $50 \mathrm{~mm} \mathrm{Hg}$ and by a certain amount of vapor instability which is partially blamed on the adsorption occurring in the channels and the measuring cavity. The probable error is around a few percent.

Figure 29 presents the results as a function of pressure for the three frequencies: $7.25,9.20$ and $11.5 \mathrm{Gc} / \mathrm{s}$. The shapes of the other curves $(\alpha, p)$ for other frequencies within the range 7 to $12 \mathrm{Gc} / \mathrm{s}$ fall well within the limits of the curve shown on figure 29 . Gennaoui [1949, 1950, and 1951] is, to our knowledge, the only other worker who has made this type of measurement on chloroform. His results at $\nu=9.45 \mathrm{Gc} / \mathrm{s}$ and at $t=20{ }^{\circ} \mathrm{C}$ are in good agreement with our results (fig. 29). For pressures of 50 and $100 \mathrm{~mm} \mathrm{Hg}$ the absorption as a function of frequency is presented on figure 30 .

Since, in the literature, there exists no other results to complete ours, the interpretation of our

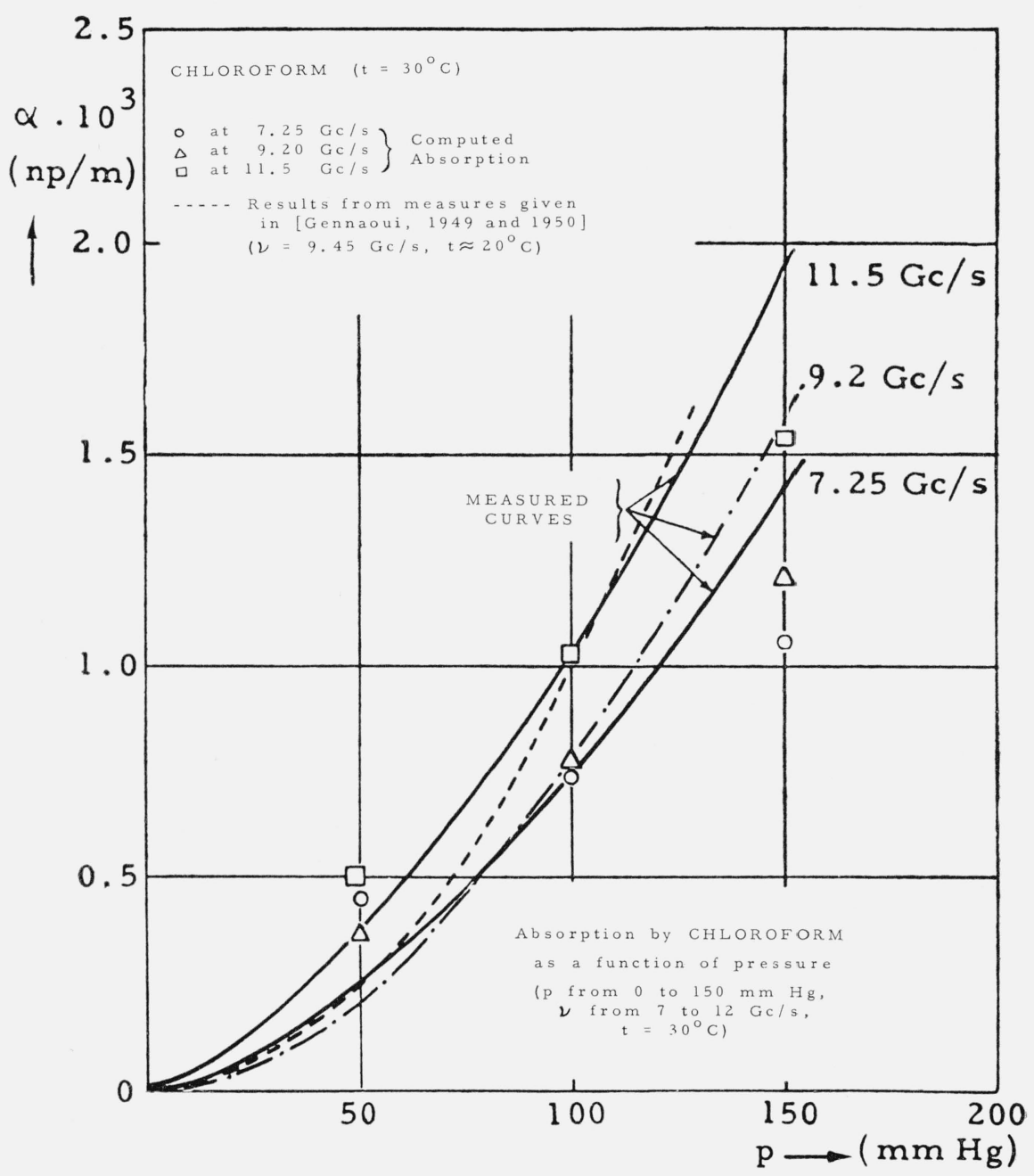

Figure 29. Chloroform absorption as a function of the pressure. 
measurement, should suggest two hypotheses. Or the measured absorption is caused mainly by the superimposed effects of the first two lines or, these first two lines are relatively weak and the measured absorption results principally from residual absorption of the higher lines.

Let us consider the first hypothesis and let us examine the measurements of absorption as a function of frequency at $p=100 \mathrm{~mm} \mathrm{Hg}$ (fig. 30). It is then possible to determine by a trial and error method two lines of the type $\mathrm{VV}-\mathrm{W}$ :

$\alpha_{J}=g_{J} \nu^{2}(\Delta \nu)_{J}\left(\frac{1}{\left(\nu_{J}-\nu\right)^{2}+(\Delta \nu)_{J}^{2}}+\frac{1}{\left(\nu_{J}+\nu\right)^{2}+(\Delta \nu)_{J}^{2}}\right)$

$$
\cdot 10^{-6},(\mathrm{np} / \mathrm{m})
$$

$\left(\nu_{J}, \nu\right.$, and $\Delta \nu$ in $\mathrm{Gc} / \mathrm{s}, J=0 \rightarrow 1$ and $\left.1 \rightarrow 2\right)$ such that their sum will give the measured value of the absorp- tion. One obtains :

for

$\nu_{01}=6.6 \mathrm{Gc} / \mathrm{s}: g_{01}=36.8$ and $(\Delta \nu)_{01}=2.8 \mathrm{Gc} / \mathrm{s}$ for

$\nu_{12}=13.2 \mathrm{Gc} / \mathrm{s}: g_{12}=16.1$ and $(\Delta \nu)_{12}=3.0 \mathrm{Gc} / \mathrm{s}$

The corresponding lines and their sum are plotted on figure 30 from which one can obtain good agreement with the experiment.

This agreement breaks down, however, when we try to adjust these lines with the results obtained

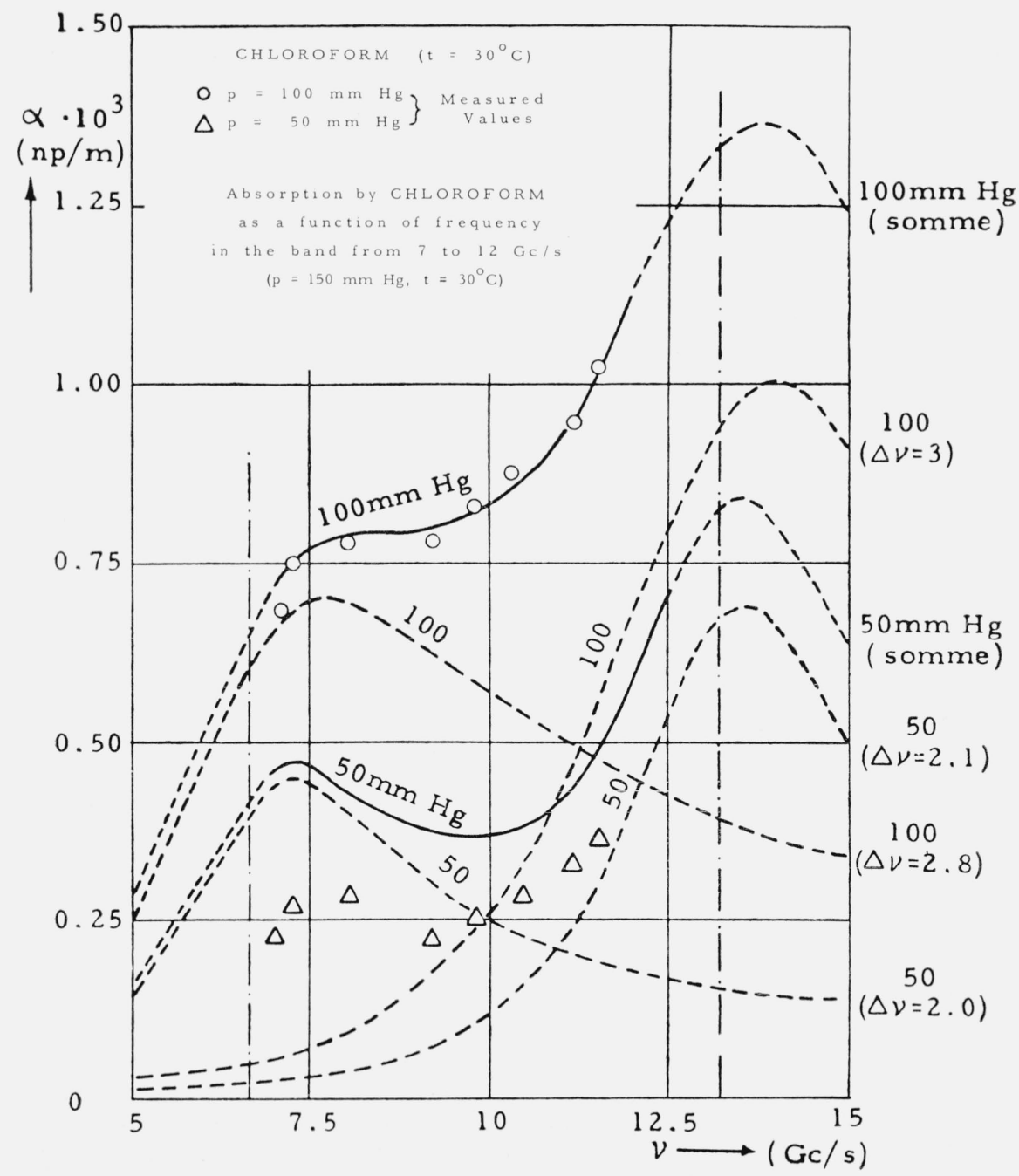

Figure 30. Attempt at the analysis of the chloroform spectrum. 
at $p=50 \mathrm{~mm} \mathrm{Hg}$. The sum of the effects of the two lines, if we admit that the parameters $\Delta \nu$ remain proportional to the pressure, gives a value for the absorption sometimes greater than the measured value. One is tempted to suggest a variation of $\Delta \nu$ which is not proportional to the pressure, although appreciable deviation from proportionality seems improbable. This discrepancy with the experimental results will remain even if one takes $(\Delta \nu)_{01}=2.0$ and $(\Delta \nu)_{12}=2.1 \mathrm{Gc} / \mathrm{s}$ at $p=50$ $\mathrm{mm} \mathrm{Hg}$. These lines have been drawn on figure 30 and their sum is still from 30 to 100 percent greater than the measured absorption.

If one supposes that $\Delta \nu$ varies with the gas pressure according to a law indicated by the value adopted above, the very shape of the curves $(\alpha, p)$ would be different from those of the experimental curve; this is indicated by the positions of the "calculated points" on figure 29.

The shapes of all the measured curves which are parabolas of increasing slope indicate that the absorption (around $7 \mathrm{Gc} / \mathrm{s}$ in particular) is not very much influenced by the closeness of an intense resonance line. The slope of the $(\alpha, p)$ curves should have a large value at weak pressure and decrease later on.

We will now consider the second hypothesis suggested above. This requires a theoretical estimate of the expected intensity of a large number of the first line of the rotational spectrum of chloroform. We must compute the expression (see sec. $2.3)$ :

$$
g_{J}=\frac{2 \pi N}{3 c \epsilon_{0} k T} f_{J}\left|\mu_{J}\right|^{2},
$$

If one replaces the universal constants by their values and if one supposes that as far as $N$ is concerned (number of molecules per unit volume) the gas obeys the law of a perfect gas (see sec. 2.1) we have:

$$
\frac{2 \pi N}{3 c \epsilon_{0} k T}=6 \cdot 10^{47} \text { at } t=30^{\circ} \mathrm{C} \text { and } p=100 \mathrm{~mm} \mathrm{Hg} .
$$

The fraction of molecules $\left(f_{J K}\right)$ which are in a given state of rotation $(J, K)$ is given, for a symmetrical molecule by the relation [Townes and Schalow, 1955 (ch. 3)]:

$$
\begin{array}{r}
f_{J K}=\frac{(2 J+1) \exp \left\{-\left[B J(J+1)+(C-B) K^{2}\right][h / k T]\right\}}{\left[\sum_{J=0}^{\infty} \sum_{K=-J}^{J}(2 J+1) \exp \{-[B J(J+1)\right.} . \\
\left.\left.\left.+(C-B) K^{2}\right][h / k T]\right\}\right]
\end{array}
$$

In this expression, the numerator is proportional to the probability of population of the state $(J, K)$ given by Boltzmann Law while the denominator is the partition function. The factor $(2 J+1)$ is the statistical weight which takes into account the different orientations $(M=j, j-1, \ldots, 0, \ldots$, $-J)$ of the $J$ vector in space. $B$ is the usual rotational constant of the molecule $\left(B=3.3 \times 10^{9} \mathrm{c} / \mathrm{s}\right.$ for chloroform) and $C=h / 8 \pi^{2} I_{c}$ must be computed using the moment of inertia of the molecule with espect to its axis of symmetry. From the structural rata of the chloroform molecule (fig. 23) one obtains:

$I_{c}=2 M_{c l} l_{12}^{2}(1-\cos \theta)=496 \cdot 10^{-47} \mathrm{~kg} \cdot \mathrm{m}^{2}$, whence:

$$
C=1.7 \cdot 10^{9} \mathrm{c} / \mathrm{s} \text {. }
$$

$B h$ and $C h$ are small with respect to $k T$ and the sum appearing in the denominator of (3.29) can be replaced by an integral. The result obtained in this fashion is then multiplied by two to take account of the statistical weights of the spins as well as the inversion which was not included in expression (3.29). The exponential factor can be neglected in the final result; it is indeed smaller than unity by 1.5 percent in our case where $J=5$. Thus one obtains:

$$
f_{J K}=f_{J}=2(2 J+1) \sqrt{\frac{B^{2} C h^{3}}{\pi(k T)^{3}}}=9.67 \cdot(2 J+1) \cdot 10^{-6} .
$$

This quantity should be multiplied by the fraction $f_{v}$ of molecules which are in the vibrational state corresponding to the considered transition. We have assumed that $f_{v}$ is approximately 1 .

Finally, we must compute the matrix element of the dipole moment $\left|\mu_{\jmath}\right|$. We make use of relation (3-10 of reference [Townes and Schalow, 1955]) and sum it over all quantic numbers $K=0, \pm 1$, $\ldots, \pm J$. This gives

$$
\left|\mu_{J}\right|^{2}=\frac{1}{6} \mu^{2} \frac{(4 J+3)(J+2)}{(2 J+1)},
$$

$\mu$ being the classic dipole moment of the molecule whose value is [Townes and Schalow, 1955]:

$$
\begin{aligned}
\mu=1.2 \text { debye }=1.2 \cdot 3.3356 \cdot 10^{-30} \mathrm{Cb} \cdot \mathrm{m} \\
=4.003 \cdot 10^{-30} \mathrm{Cb} \cdot \mathrm{m} .
\end{aligned}
$$

The VV-W formula can then be applied for $t=30^{\circ} \mathrm{C}$ and $p=100 \mathrm{~mm} \mathrm{Hg}$ under the form:

$$
\left(\alpha_{J}\right)_{\max } \approx 6 \cdot 10^{56} \cdot f_{J}\left|\mu_{J}\right|^{2} \frac{\nu^{2}}{(\Delta \nu)_{J}},(\mathrm{np} / \mathrm{m}),
$$

$f_{j}$ and $\left|\mu_{J}\right|^{2}$ are given by (3.31), (3.32), (3.33) and $\nu$ and $\Delta \nu$ are expressed in $\mathrm{Gc} / \mathrm{s}$. The maxima of intensity of the early lines computed in the above fashion are given in table 6 . The width parameter $\Delta \nu$ has been taken as $2 \mathrm{Gc} / \mathrm{s}$ for all lines which introduce a rather crude approximation.

The maximum intensity of the lines should increase rapidly with $J$ as long as the exponential appearing in (3.29) does not begin to decrease too quickly. However, the maximum intensity of the line $J=7 \rightarrow 8$ 
is of the order of $6 \times 10^{-3}$ neper $/ \mathrm{m}$, at $\nu \approx 55 \mathrm{Gc} / \mathrm{s}$ while its contribution at $10 \mathrm{Gc} / \mathrm{s}$ is, according to (3.25) of the order of $0.001 \times 10^{-3}$ neper $/ \mathrm{m}$. Consequently, it is hard to believe that the total measured absorption which is of the order of $1 \times 10^{-3}$ neper $/ \mathrm{m}$ in the band 7 to $12 \mathrm{Gc} / \mathrm{s}$, could be produced in its totality by the "residual absorption," sum of all the contribution of all the higher lines. This remains true even if one bears in mind that the $\mathrm{VV}-\mathrm{W}$ formula predicts along the lower wings of the lines a value of the absorption smaller than the true value.

In spite of the small number of frequencies selected for the experiment and in spite of the approximations introduced in the theoretical computations, we conclude that the measured absorption is produced partially by the first two lines, which have appreciable intensity, and in part by the residual absorption of the higher lines.

TABLE 6

\begin{tabular}{|c|c|c|c|c|c|c|c|c|}
\hline$J=$ & 0 & 1 & 2 & 3 & 4 & 5 & 6 & 7 \\
\hline$\nu_{J}(\mathrm{Gc} / \mathrm{s})=$ & 6.6 & 13. 2 & 19.8 & 26.4 & 33.0 & 39.6 & 46.2 & 52.8 \\
\hline$\left.\alpha_{J}\right)_{\max } \cdot 10^{3}$, & 0.002 & 0.028 & 0.135 & 0.405 & 0.96 & 1.96 & 3.57 & 5.98 \\
\hline$\left(\alpha_{J} / \alpha_{0}\right)_{\max }=$ & 1 & 14 & 67 & 202 & 480 & 980 & 1785 & 2990 \\
\hline
\end{tabular}

D. The influence of temperature on the absorptionThe gas temperature influences the phenomenon of absorption in a rather complicated fashion. Indeed, several of the quantities appearing in the VV-W (2.33) are functions of the temperature. The number $N$ of molecules per unit volume can be considered as proportional to $p / T(2.3)$. On the other hand, although the matrix element of the dipole moment $\left|\mu_{i j}\right|$ is temperature independent, the fraction $b_{i j}$ depends on it. We saw in a previous section that in the case of chloroform, and for a given "isolated" line of a symmetric molecule and for transition of low quantic number $J$, this fraction is proportional to $T^{-3 / 2}$. Under those conditions and if both pressure and frequency are fixed:

$$
\alpha=\left(C^{t e}\right) \cdot T^{-3.5} \cdot F
$$

The factor $F$ of the $\mathrm{VV}-\mathrm{W}$ formula contains the width parameter $\Delta \nu$ which itself depends on the temperature.

If one adopts the approximation of the Kinetic Theory of Gases where the molecules are approximated by small rigid spheres and where the cross section is necessarily independent of velocity, we will have $\Delta \nu \sim T^{-1 / 2}$ (see sec. 2.3B) [Bleaney and Penrose, 1948b]. The more advanced theories of shock broadening indicate implicitly that $\Delta \nu$ should be proportional to $T^{-1}$ [Anderson, 1949c; Howard and Smith, 1950; Leslie, 1951; Margenau, 1949a and 1949b; Margenau and Watson, 1936; Mizushima, 1951; 'Townes and Schalow, 1955]. Since $F \approx\left(C^{t e}\right)$ ' $\Delta \nu$ in the neighborhood of resonance line while $F \sim\left(C^{t e}\right) \Delta \nu$ on the far wings of a line (see sec. $2.3 \mathrm{C}$ ) the theoretical predictions for the present case can be resumed in table 7 .

We have studied experimentally the influence of the temperature on the absorption by chloroform. The results shown on figure 31 for $\nu=9.33 \mathrm{Gc} / \mathrm{s}$ and $p=50 \mathrm{~mm} \mathrm{Hg}$ indicate that the temperature influences the absorption according to a $(273 / T)^{4}$ law. This result cannot be compared directly with the above theoretical predictions which were subjected to certain hypotheses, one of them requiring that the absorption was produced by one single isolated line. The measured absorption, is on the contrary, produced by many lines (see sec. 3.2C). Nevertheless at a frequency of $9.33 \mathrm{Gc} / \mathrm{s}$ one is located on the wings of the absorbing lines, and we can state that within the limits of the complexity of the phenomenon and within the precision of the measurements, the experimental values are well within the theoretical predictions.

The case of ammonia is different. The absorption studied as a function of temperature, at the same frequency of $9.33 \mathrm{Gc} / \mathrm{s}$ is here caused by all the inversion lines of ammonia which are now lumped into one single line by the broadening effects. It appears then that the sum:

$$
\sum_{J K} f_{J K}\left|\mu_{J K}\right|^{2}\left(\frac{1}{\left(\nu_{J K}-\nu\right)^{2}}+\frac{1}{\left(\nu_{J K}+\nu\right)^{2}}\right)
$$

which appears in the VV-W formula, when one is sufficiently far from resonance, is practically independent of temperature [Birnbaum and Maryott, 1953a and 1953b]. Consequently, we have in accordance with the statements made previously (see also reference [Krishnaji and Swarup, 1953a and $1953 \mathrm{~b}$, and 1954]):

$\alpha=\left(C^{t e}\right) \cdot T \cdot \cdot^{-2} \cdot(\Delta \nu)$,

(for fixed pressure and frequencies),

where $\Delta \nu$ is proportional either to $T^{-1 / 2}$ or $T^{-1}$ according to the chosen theory.

\begin{tabular}{|c|c|c|c|c|}
\hline \multirow[b]{2}{*}{$\begin{array}{l}\Delta \nu \text { proportional to } \\
\alpha \text { proportional to }\end{array}$} & \multicolumn{2}{|c|}{ for $\alpha_{\max }$} & \multicolumn{2}{|c|}{ for $\alpha_{\text {wing }}$} \\
\hline & $\begin{array}{l}T^{-1 / 2} \\
T^{-3}\end{array}$ & $\begin{array}{l}T^{-1} \\
T^{-2.5}\end{array}$ & $\begin{array}{l}T^{-1 / 2} \\
T^{-4}\end{array}$ & $\begin{array}{l}T^{-1} \\
T^{-4.5}\end{array}$ \\
\hline
\end{tabular}

$$
\text { TABLe } 7
$$

The experiment whose results for $\nu=9.33 \mathrm{Gc} / \mathrm{s}$ and $p=160 \mathrm{~mm} \mathrm{Hg}$ are presented in figure 32 indicates with a remarkable precision that the ammonia absorption obeys a $(273 / T)^{2 \cdot 5}$ law. The ratio $\alpha_{T} / \alpha_{273}$ remains practically constant for all pressures in the interval considered, specifically from 30 to $250 \mathrm{~mm} \mathrm{Hg}$. From this result and from (3.37) one deduces that the width parameter $\Delta \nu$ of the unified line of ammonia will vary, at average pressures, as $T^{-1 / 2}$ in accordance with the Kinetic Theory of Gases.

If this conclusion agrees with the previous results 


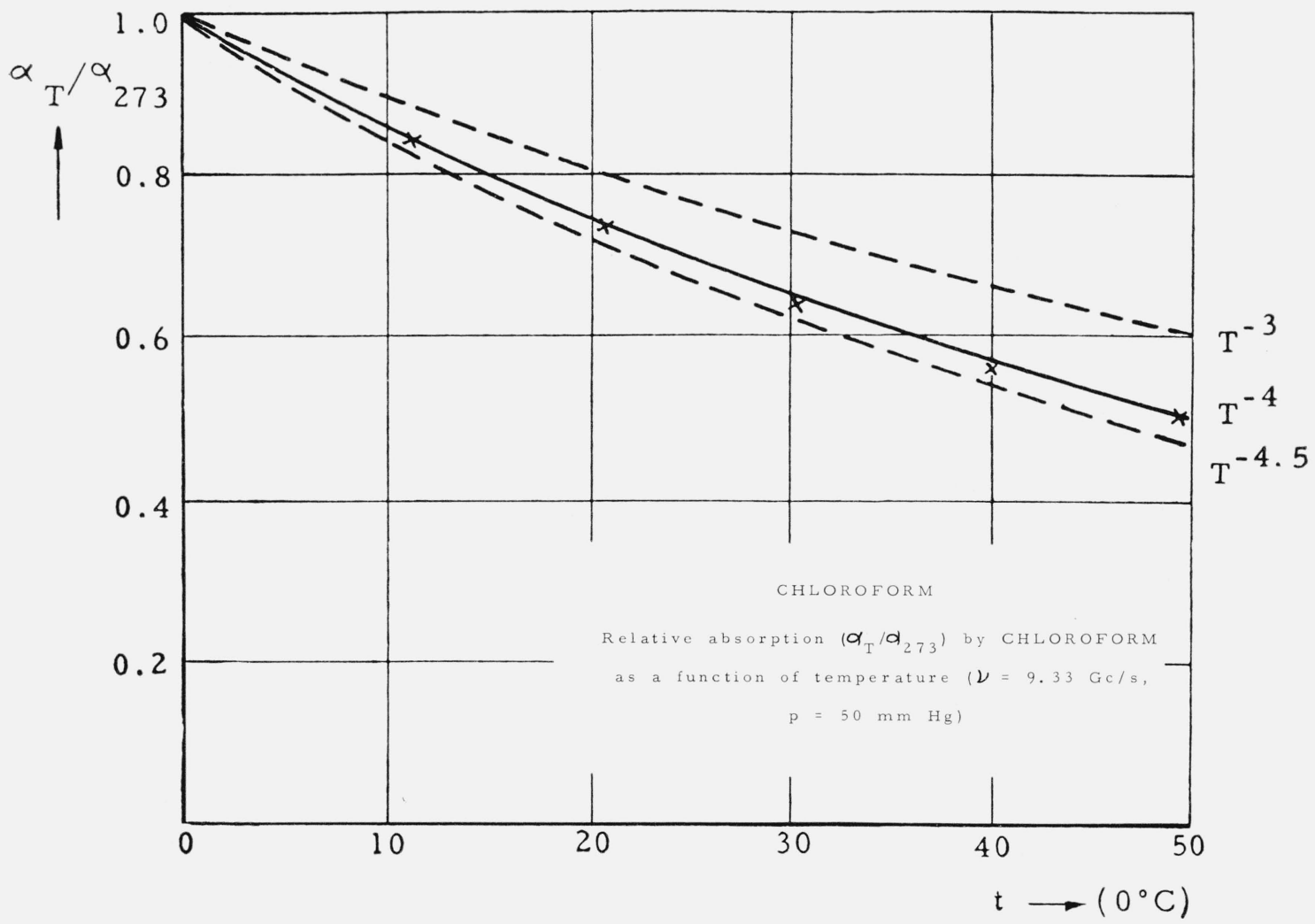

FiguRE 31. Influence of the temperature (chloroform).

of Krishnaji and Swarup [1953a and 1953b, and 1954], and Swarup [1954], it disagrees with the work of Birnbaum and Maryott who found $\alpha \sim(273 / T)^{3}$ and thus $\Delta \nu \sim T^{-1}$ [Birnbaum and Maryott, 1953a and $1953 \mathrm{~b}$, and 1954]. Without raising question as to the precision of the experimental setup of these authors, it appears to us that the curves $(\alpha, p)$ obtained by us with good reproducibility and high precision in the interval 0 to $50{ }^{\circ} \mathrm{C}$ establish the validity of the law $\alpha \sim(273 / T)^{2.5}$. This temperature factor has been included in (3.17) which represents then the absorption by ammonia as a function of temperature under the conditions specified here (see sec. 3.2C).

The dependence of the absorption on the temperature for ammonia could be considered analogous to that of oxygen [Van Vleck, 1947] (see also sec. 3.2A).

E. Dispersion - The index of refraction of the absorbing gas is expressed by (2.36) in accordance with the VV-W theory. This expression, when applied to the case of ammonia where the absorption can be approximated by a single line, becomes (MKS units) :

where

$$
n-1=A \cdot \frac{c}{2 \pi} G+\left(C^{t e}\right) p,
$$

$$
G=\frac{1}{2}\left[\frac{\nu_{0}\left(\nu_{0}+\nu\right)+(\Delta \nu)^{2}}{\left(\nu_{0}+\nu\right)^{2}+(\Delta \nu)^{2}}+\frac{\nu_{0}\left(\nu_{0}-\nu\right)+(\Delta \nu)^{2}}{\left(\nu_{0}-\nu\right)^{2}+(\Delta \nu)^{2}}\right],
$$

$$
A=\frac{2 \pi N}{3 c \epsilon_{0} k T} \sum_{J K} f_{J K}\left|\mu_{J K}\right|^{2},
$$

the term $\left(C^{t e}\right) p$ expresses the contribution of the inducad molecular polarization to the index of refraction.

The factor $A$, which also appears in the formula giving the absorption, is determined by comparison with (3.17). One obtains at $t=0{ }^{\circ} \mathrm{C}$ :

$$
A \cdot \frac{c}{2 \pi}=\frac{3 g}{2 \pi} p \cdot 10^{-6}
$$

where $p$ is measured in $\mathrm{mm} \mathrm{Hg}$ and $g$ is the absorption factor already studied in (C). Finally, one has

$$
(n-1) \cdot 10^{6}=\frac{3 q}{2 \pi} p G+\left(C^{t e}\right) \cdot p,
$$

where $G$ is computed by means of (3.39) where $\nu_{0}, \nu, \Delta \nu$, are measured in $\mathrm{Gc} / \mathrm{s}$ ( $G$ is a shape factor which is dimensionless). For these computations the values of $\nu_{0}$ and $\Delta \nu$ are those previously determined in function or the pressure (fig. 28). This allows us to judge if a formula of the $\mathrm{VV}-\mathrm{W}$ type adapted for the best representation of the experimental measurements of the absorption is also capable to represent dispersion phenomena. Dur- 


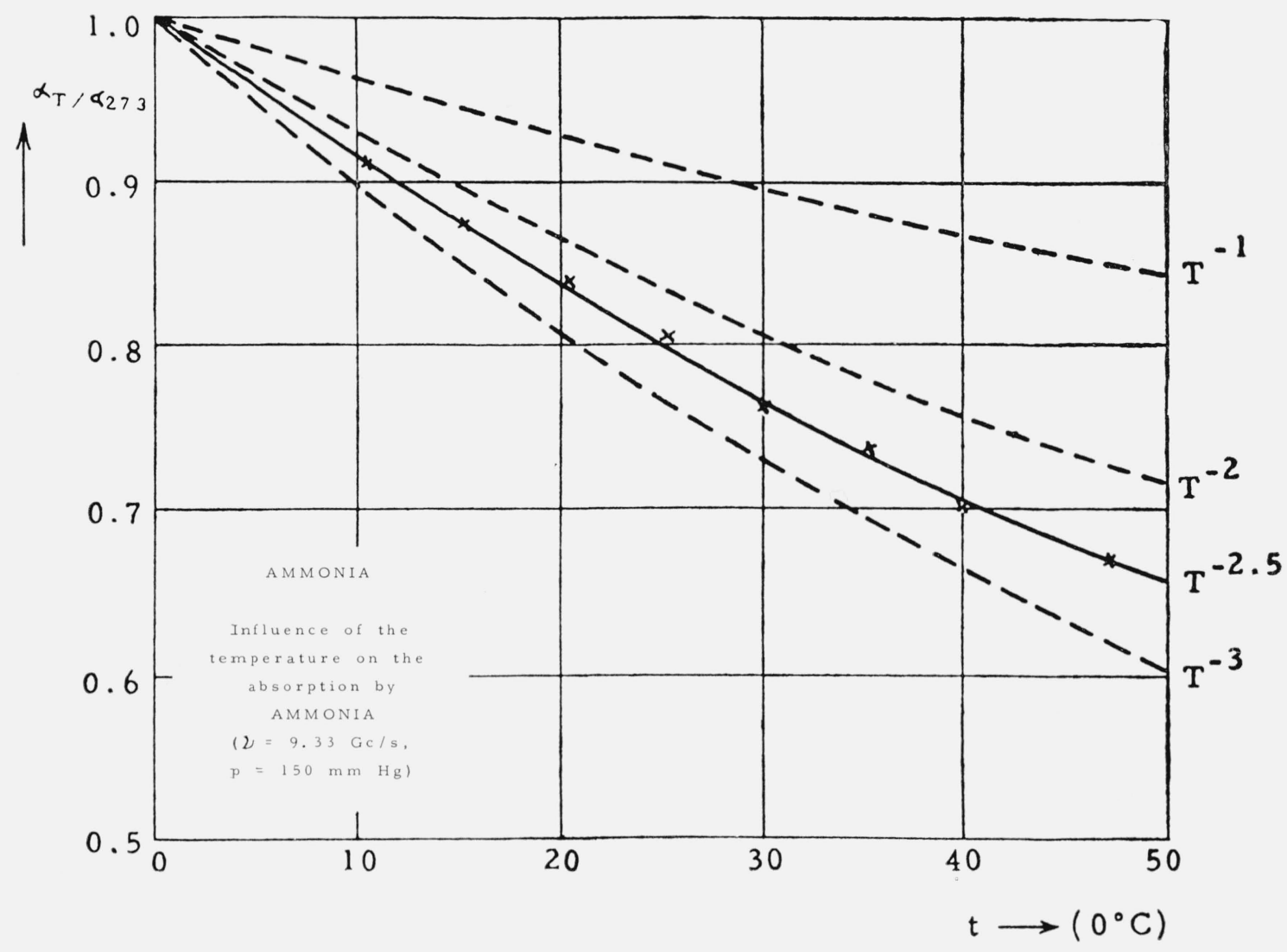

Figure 32. Influence of the temperature (ammonia).

ing the actual computation we have also supposed that the factor $g(3.17)$ has a constant value of 3.3 .

The index $(n-1) \times 10^{6}$ has been computed as a function of the pressure for the frequencies 9.33 and $11.1 \mathrm{Gc} / \mathrm{s}$. The slope $\partial(n-1) \times 10^{6} / \partial p$ is then computed and plotted on figure 33 as a function of the pressure. A sufficient precision must be maintained during the computation, the fourth significant figure can still influence the results.

The slope measured as a function of the pressure at those same frequencies is also plotted on the same figure. The measured curves have been shifted by 3.10 along the ordinate axis in order to take account of the constant term which appears in the slope in accordance with (3.42). The agreement between experiment and theory indicates that if one uses the same parameters $\nu_{0}$ and $\Delta \nu$, the absorption and the associated phenomenon of the variation of the slope of the refractive index can be expressed as a function of the pressure by $\mathrm{VV}-\mathrm{W}$ formulas with equal precision.

These results obtained from ammonia should give useful indications as to the behavior of oxygen which should be analogous (see sec. 3.2A).

The slope of the index decreases as a function of pressure when the measured frequency is located on the lower wing of the unified absorption line of ammonia, far enough from resonance. This had been mentioned by Battaglia et al. [1958] for a frequency of $9.4 \mathrm{Gc} / \mathrm{s}$. The more complete measurements given here for two frequencies and the direct comparison of these results with all other experimental data on ammonia absorption can clarify this phenomenon and help shed new lights on the consequences of the $\mathrm{VV}-\mathrm{W}$ theory. Quite recently, Heineken and Wijn [1958] have presented at the A.M.P.E.R.E. colloquium (July 1958) measurements at $50 \mathrm{Gc} / \mathrm{s}$ which indicate the inverse behavior (increase of the slope of the index with the pressure) predicted by the theory for the case where one is located sufficiently far on the upper wing of the unified line of ammonia.

As far as the values themselves of the index are concerned, although we are more interested in their dependence on the pressure, we give the measured value for ammonia along with the results from other workers in table 8. A certain amount of discrepancy exists between the results from different authors and these cannot be entirely explained by the difference in experimental conditions. Dispersion by chloroform at $30^{\circ} \mathrm{C}$ was studied in the band from 7 to $12 \mathrm{Gc} / \mathrm{s}$. The effects one tries to measure are very weak and we were using the sensitivity of the instrument to its limit. Under those conditions we should not expect a high precision (figs. 34 and 35). A discussion of the results would be complicated and almost. hopeless if one was to try to take into account the influence of a large number of lines. This discussion, 


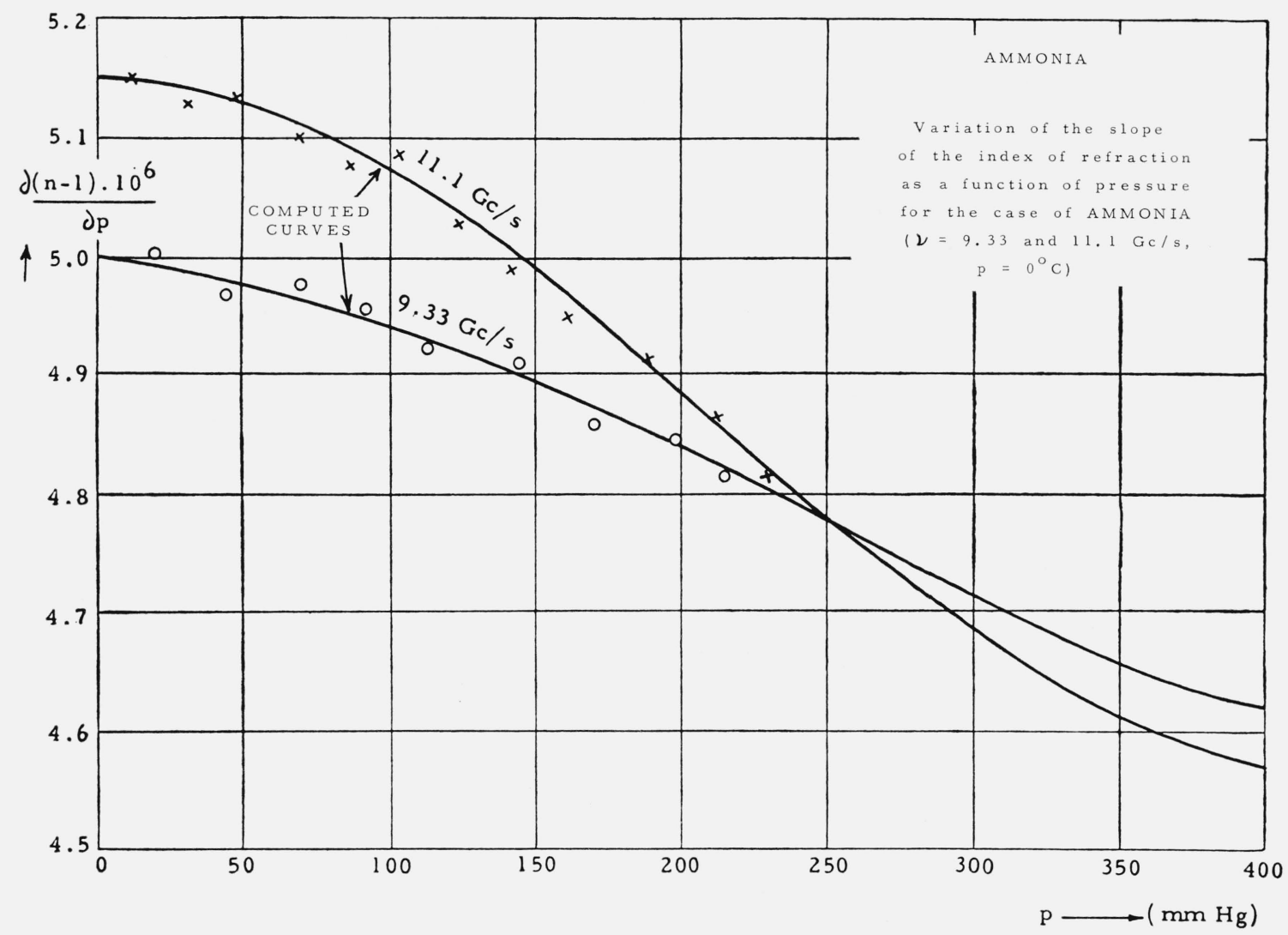

FiguRE 33. Dispersion as a function of the pressure (ammonia).

however, can be simplified and lead to general qualitative conclusions.

TABLE 8. Index of refraction of ammonia

\begin{tabular}{|c|c|c|c|c|c|}
\hline Date & Author & $\begin{array}{l}\text { Fréquence } \\
(\mathrm{Gc} / \mathrm{s})\end{array}$ & $\begin{array}{l}\text { Tempé- } \\
\text { rature } \\
\left({ }^{\circ} \mathrm{C}\right)\end{array}$ & $\begin{array}{c}\text { Pression } \\
(\mathrm{mm} \mathrm{Hg})\end{array}$ & $(n-1) \cdot 10^{6}$ \\
\hline (1946) & $\begin{array}{l}\text { (GUERIN) } \\
\text { WALTER-HERSHBER- } \\
\text { GER. }\end{array}$ & $\begin{array}{l}(5890 \text { A) } \\
(\alpha)(\text { B.F.) }\end{array}$ & $\begin{array}{c}0 \\
(\mathrm{amb} .)\end{array}$ & $\begin{array}{l}760 \\
760\end{array}$ & $\begin{array}{r}376 \\
3150\end{array}$ \\
\hline 1946 & $\begin{array}{l}\text { (B) } \\
\text { WÄLTER-HERSHBER- } \\
\text { GER. }\end{array}$ & $\begin{array}{l}\text { (B.F.) } \\
9.4\end{array}$ & $\begin{array}{r}17.8 \\
(\mathrm{amb} .)\end{array}$ & $\begin{array}{l}760 \\
760\end{array}$ & $\begin{array}{l}3150 \\
2650\end{array}$ \\
\hline 1946 & $\begin{array}{l}\text { VAN ITTERBEEK-DE } \\
\text { CLIPPELEIER }\end{array}$ & (B.F.) & $\begin{array}{r}(\mathrm{amb} .) \\
30 \\
(0-120)\end{array}$ & $\begin{array}{l}760 \\
760\end{array}$ & $\begin{array}{l}2750 \\
2703\end{array}$ \\
\hline $\begin{array}{l}1950 \\
1950\end{array}$ & $\begin{array}{l}\text { MURPHY-RAYMOND } \\
\text { BIRNBAUM }\end{array}$ & $\begin{array}{r}9.4 \\
2.8 \\
9.27\end{array}$ & $\begin{array}{r}26 \\
25\end{array}$ & $\begin{array}{r}(\tau)(760) \\
760 \\
760\end{array}$ & $\begin{array}{l}3540 \\
3025 \\
\end{array}$ \\
\hline 1951 & GENNAOUI & 9.45 & 17.8 & $\begin{array}{l}760 \\
760\end{array}$ & 3085 \\
\hline 1956 & $\begin{array}{l}\text { GOZZINI-B ATTAGLIA- } \\
\text { B RUIN. }\end{array}$ & 9.4 & $\begin{array}{l}0 \\
0\end{array}$ & $\begin{array}{r}300 \\
(0)(760)\end{array}$ & $\begin{array}{l}1350 \\
(3420)\end{array}$ \\
\hline 1958 & présent work & $\begin{array}{l}9.33 \\
11.1\end{array}$ & $\begin{array}{l}0 \\
0 \\
0 \\
0\end{array}$ & $\begin{array}{c}300 \\
\left({ }^{(\delta)}(760)\right. \\
300 \\
\left({ }^{\delta}\right)(760)\end{array}$ & $\begin{array}{l}1465 \\
(3570) \\
1485 \\
(3595)\end{array}$ \\
\hline
\end{tabular}

${ }^{\alpha}$ Computed by the authors, using the Debye-Langevin formula.

$\beta$ Values borrowed from the "In ternational Critical Tables" and reduced to the indicated conditions by means of [Gennaoui, 1949 and 1951].

$\tau$ Reduced from 730 to $760 \mathrm{~mm}$ Hg assuming $(n-1) p$. for pressures greater than one half of $1 \mathrm{~atm}$.

First, we make the simplifying assumption that dispersion is caused principally by the first two lines of the rotation spectrum. This allows us to write the index of refraction in accordance with the $\mathrm{VV}-\mathrm{W}$ theory by a formula of the type:

$(n-1) \cdot 10^{6} \approx B_{1} \cdot\left(G_{1}-1\right)+B_{2}\left(G_{2}-1\right)+\left(C^{t e}\right) \cdot p$.

$G_{1}$ and $G_{2}$ are given by (3.39) where one substitutes for $\nu_{0}$ the quantity $\nu_{01}=6.6 \mathrm{Gc} / \mathrm{s}$ for the first line and the quantity $\nu_{12}=13.2 \mathrm{Gc} / \mathrm{s}$ for the second line. We take for the width parameter $\Delta \nu$ the following value:

$$
(\Delta \nu)_{01}=2.7 \mathrm{Gc} / \mathrm{s} \text { and }(\Delta \nu)_{12}=3.0 \mathrm{Gc} / \mathrm{s}
$$

with a pressure of $p=100 \mathrm{~mm} \mathrm{Hg}$.

The factors $B_{1}$ and $B_{2}$ are determined by the method outlined at the beginning of this section where use is made of absorption data $((3.25)$ to (3.27)). One finds $B_{1}=1.76$ and $B_{2}=0.77$.

The graphs giving the results of those computations and displaced by a constant amount indicated by experience to be 295 , are plotted on figure 34 . The all important experimental conclusion is that this quantity varies in a sense predicted by the theory.

This study of the variation of the slope of the index of refraction as a function of the pressure at fixed temperature and frequency $(\nu=11.5 \mathrm{Gc} / \mathrm{s}$ and $t=30^{\circ} \mathrm{C}$ ) has been accomplished in the following fashion. First, we have supposed that for given frequencies the influence of the first line was weak 


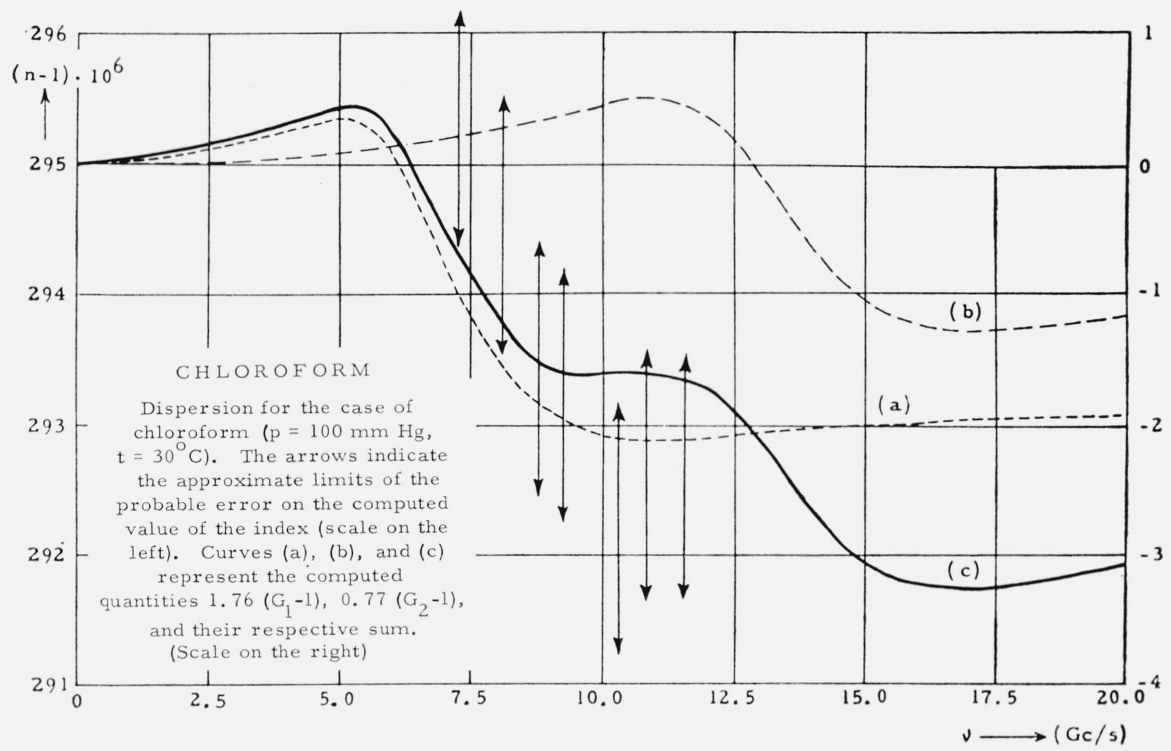

Figure 34. Attempt at a study of the dispersion for the case of chloroform. (Index vs frequency)

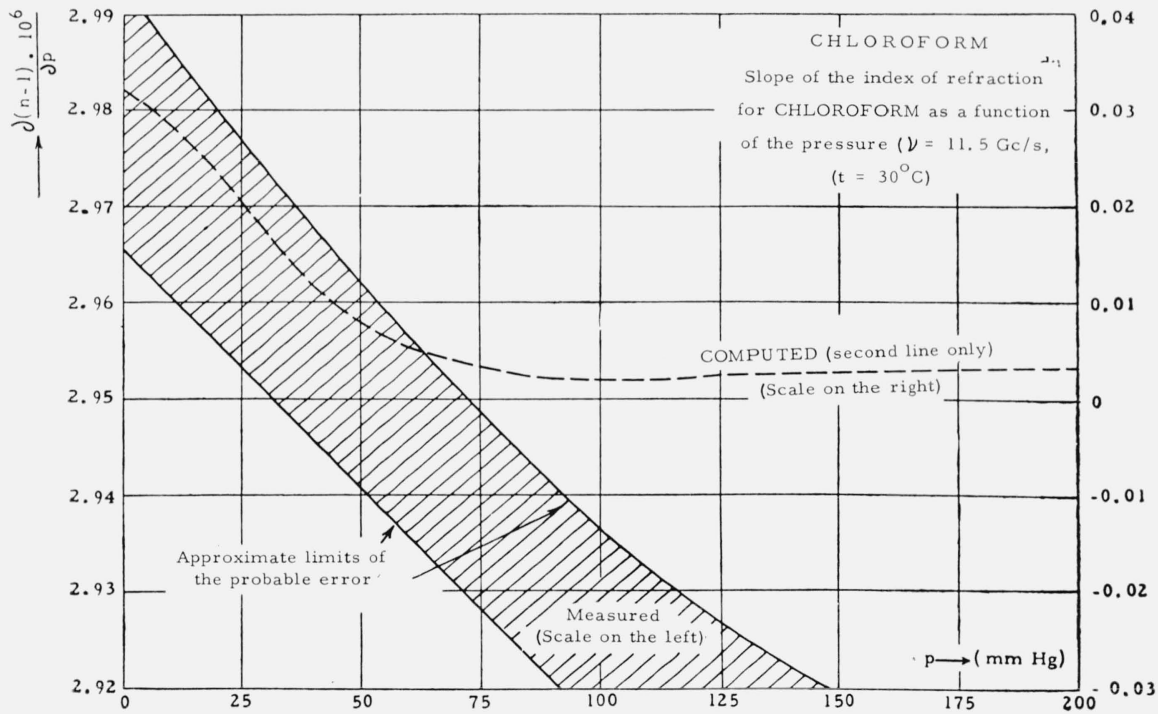

FIgure 35. Attempt at a study of the dispersion for the case of chloroform. (Slope vs pressure)

when compared to that of the second. The influence of the high line which should increase the effect of the second line has been discarded. Hoping to underline the sense of variation of this phenomenon we write:

$$
\frac{\partial(n-1) \cdot 10^{6}}{\partial p}=\frac{\partial\left\{B_{2}\left(G_{2}-1\right)\right\}}{\partial p}+\left(C^{t e}\right)
$$

Let us assume that $B_{2}(=0.77$ at $p=100 \mathrm{~mm} \mathrm{Hg})$ which contains the number $N$ of molecules per unit volume and which is proportional to the pressure be given by the relation $B_{2}=0.77 \times 10^{-2} p$. If one also assumes that the width parameter $(\Delta \nu)_{12}$ equals $3 \mathrm{Gc} / \mathrm{s}$ at $p=100 \mathrm{~mm} \mathrm{Hg}$ and remains proportional to the pressure $\left((\Delta \nu)_{12}=3 \times 10^{-2} p\right)$, one will have:

$$
\frac{\partial(n-1) \cdot 10^{6}}{\partial p}=0.77 \cdot 10^{-2}\left(G_{2}+p \frac{\partial G_{2}}{\partial p}\right)+\left(C^{t e}\right),
$$

with

$$
G_{2}=\frac{1}{2}\left[\frac{325.5+9 \cdot 10^{-4} p^{2}}{610+9 \cdot 10^{-4} p^{2}}+\frac{22.44+9 \cdot 10^{-4} p^{2}}{2.89+9 \cdot 10^{-4} p^{2}}\right] .
$$

The curve computed with the help of this formula has been plotted on figure 35 along with the experimental results. The more rapid fall of the measured slope as a function of the pressure could be due to the influence of the high lines which must contribute 


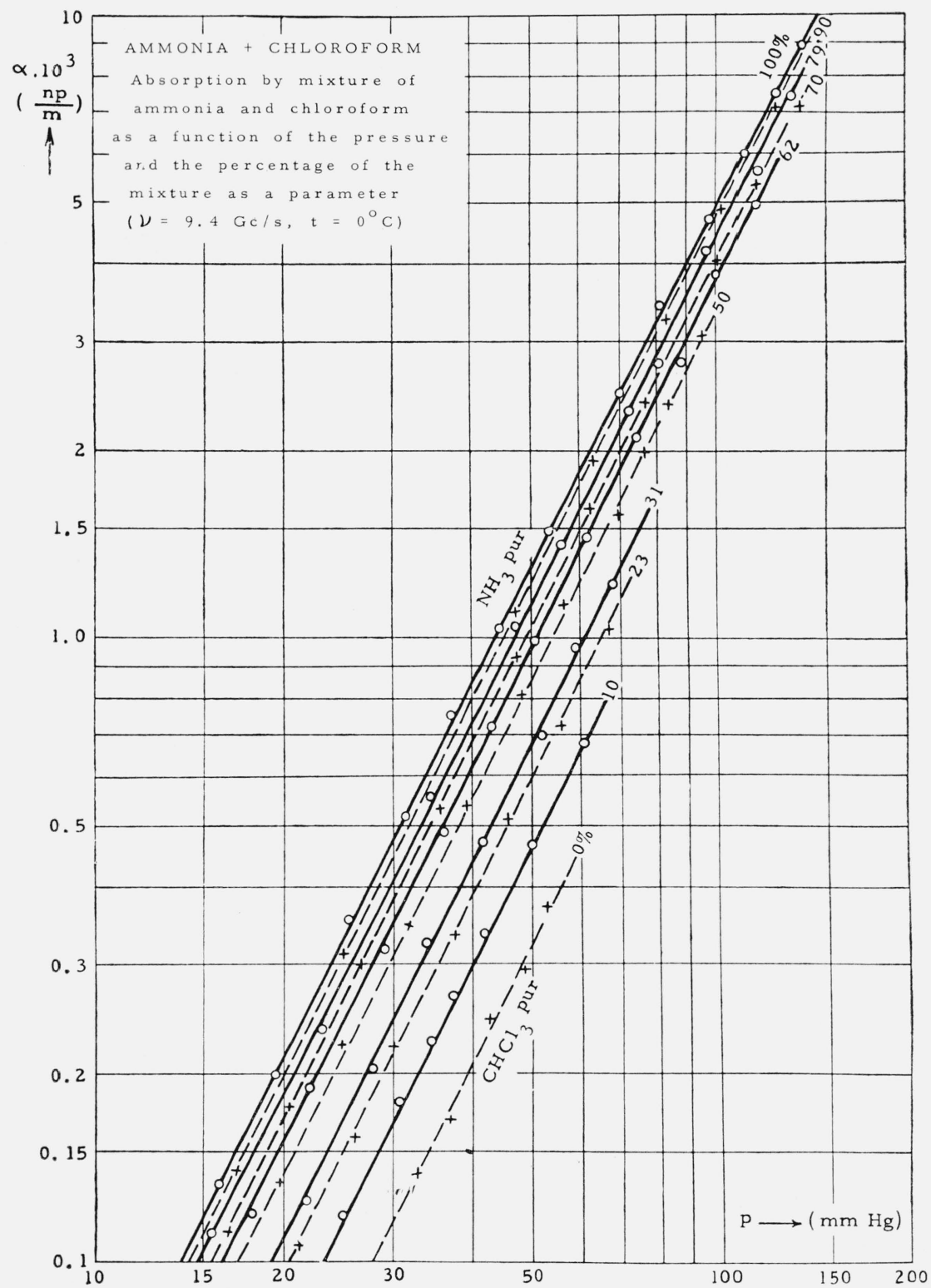

Figure 36. Absorption by mixtures of ammonia and chloroform.

to such a variation of the slope. The fall has been less noticeable for measurements made at lower frequencies and around $8 \mathrm{Gc} / \mathrm{s}$ the slope is almost constant.

The measured value of the index for chloroform is $(n-1) \times 10^{6}=293$ at $p=100 \mathrm{~mm} \mathrm{Hg}$ and $t=30{ }^{\circ} \mathrm{C}$ and $\nu=9$ to $11 \mathrm{Gc} / \mathrm{s}$. From among the results quoted in the literature we mention the values measured by Gennaoui [1951] $(n-1) \times 10^{6}=310$ at $100 \mathrm{~mm} \mathrm{Hg}$, $22{ }^{\circ} \mathrm{C}$, and $9.4 \mathrm{Gc} / \mathrm{s}$, which corresponds to a value of 294 at $100 \mathrm{~mm} \mathrm{Hg}$. All the results are in good agreement.

F. Absorption by mixture of gases-Finally, we have studied the absorption of a few mixtures of gases, ammonia + chloroform, ammonia + argon. Those mixtures were prepared in separate containers and their respective concentration evaluated by the partial pressure of their constituents. We could not avoid a certain amount of instability in these mixtures, mainly with chloroform, and the total precision is of a faw percent.

The absorption curves as a function of the pressure for the mixtures, ammonia + chloroform (at $0{ }^{\circ} \mathrm{C}$ and $\nu=9.4 \mathrm{Gc} / \mathrm{s}$ ), constitute on log-log paper an array of straight and almost parallel lines (figure 36 ). The relative absorption as a function of ammonia concentration is indicated by the curve $\mathrm{CHCl}_{3}$ of figure 37. The parallelism of the absorption curve is maintained for the case, ammonia + argon, and this is why we only get the curve of relative absorption (curve A of figure 37).

Although one finds similar works in the literature 


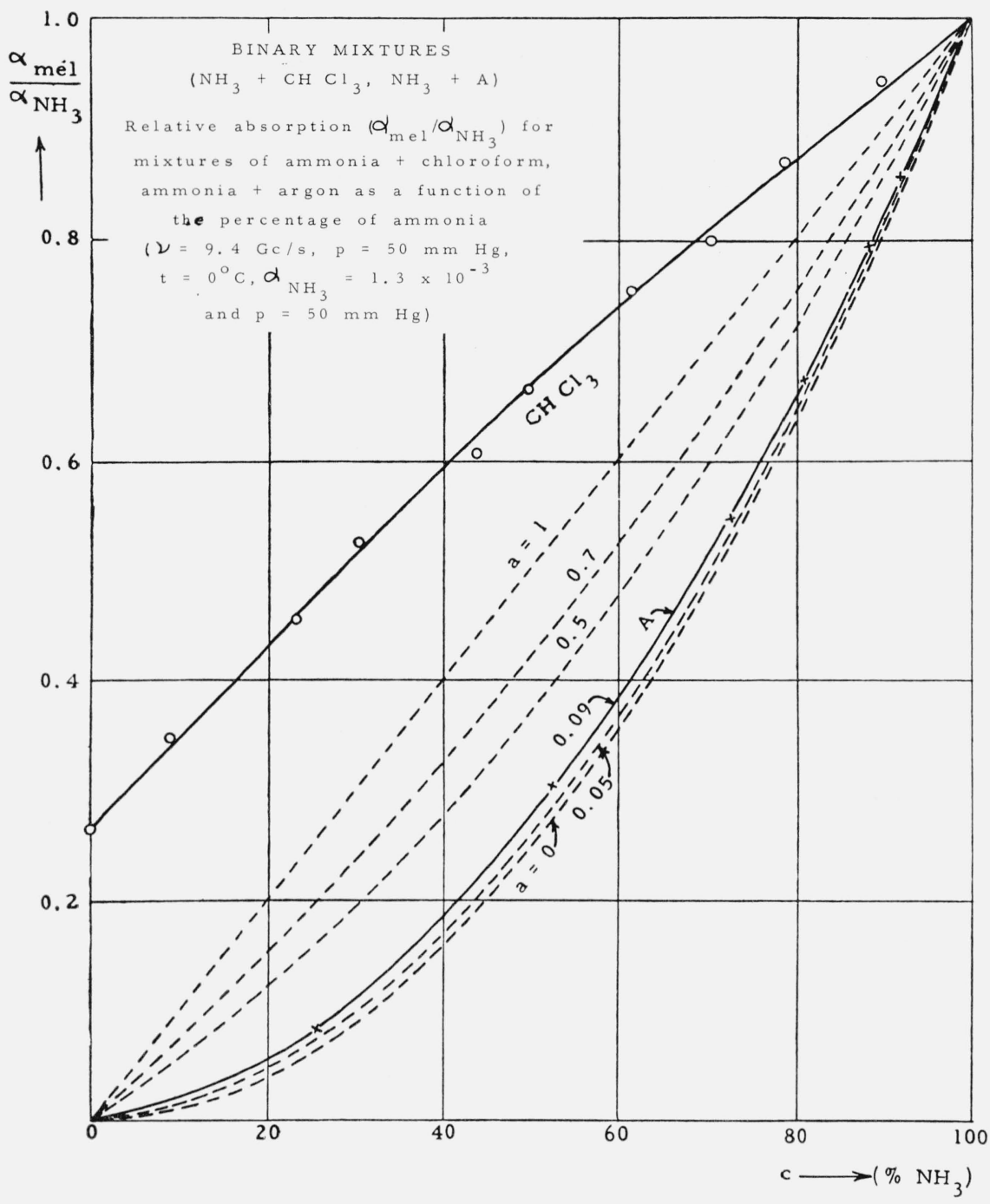

Figure 37. Relative absorption by some gaseous mixtures.

[Battaglia et al., 1958; Bleaney and Penrose, 1948b; Howard and Smith, 1950b; Smith and Howard, 1950 ], . . . these experiments are made at low pressure and are often oriented in different directions.

We can interpret our results in the following manner. Absorption by ammonia as well as by chloroform can be expressed approximately by a relation of the type:

$$
\alpha=K N \cdot(\Delta \nu),
$$

where $K$ is a constant, $N$ the number of molecules per unit of volume, and $\Delta \nu$ the line width parameter (at the measured frequency and for constant temperature and pressure).

The absorption of the mixture will be the sum of the absorptions $\alpha_{1}$ and $\alpha_{2}$ of the two constituents, the parameters $\Delta \nu$ being, however, modified by the different type of shocks which can occur in a mixture. We thus obtain for the absorption in a mixture:

$\alpha_{\mathrm{mix}}=\alpha_{1}+\alpha_{2}=K_{1} \cdot N c(\Delta \nu)_{1}+K_{2} \cdot N(1-c)(\Delta \nu)_{2}$, where $c$ is the concentration of the mixture with respect to the principal gas (ammonia). Or (see sec. $2.3 \mathrm{~B})$ :

$$
\Delta \nu=1 / 2 \pi \tau=N \bar{v}_{12} \sigma / 2 \pi,
$$

$\tau$ is the average time between two collisions, $\bar{v}_{12}$ is the average relative velocity of the molecules colliding and $\sigma$ the cross section for molecule collision. One can then write:

$$
\left.\begin{array}{l}
(\Delta \nu)_{1}=N c \bar{v}_{11} \sigma_{11}+N(1-c) \bar{v}_{12} \sigma_{12} \\
(\Delta \nu)_{2}=N(1-c) \bar{v}_{22} \sigma_{22}+N c \bar{v}_{12} \sigma_{12}
\end{array}\right\} .
$$

The meaning of indices $11,12,22$ is obvious.

Let us introduce the equations (3.50) into (3.48) and let us divide by the value of the absorption for pure ammonia (expressed by $K_{1} N^{2} \bar{v}_{11} \sigma_{11}$ ). After simplification the relative absorption $y=\alpha_{\mathrm{mix}} / \alpha_{\mathrm{NH}_{3}}$ 
is expressed by the relation:

$$
y=A c^{2}+B c+C
$$

where

$$
\left.\left.\begin{array}{l}
A=1-(1+K) a_{12}+K a_{22}, \\
B=(1+K) a_{12}-2 K a_{22}, \\
C=K a_{22},(A+B+C \equiv 1),
\end{array}\right\} \begin{array}{r}
K=K_{2} / K_{1} \\
a_{12}=\frac{\bar{v}_{12} \sigma_{12}}{\bar{v}_{11} \sigma_{11}} \\
\bar{v}_{22}=\frac{\bar{v}_{22} \sigma_{22}}{\bar{v}_{11} \sigma_{11}}
\end{array}\right\}
$$

for $c=0$ one has $y=C$ and the experiment (fig. 37) gives $C=0.27$. On the other hand $y^{\prime}(c=0)=B$ and our measurements give $B=0.83$. We thus have

$$
A=-0.10, B=0.83, C=0.27 \text {. }
$$

The resulting parabola is given by:

$$
y=-0.10 c^{2}+0.83 c+0.27
$$

and is drawn in figure 37 where one notes the good agreement with the rest of the measured values. Finally, eqs (3.52) and (3.53) give:

$$
\frac{\bar{v}_{12} \sigma_{12}}{\bar{v}_{11} \sigma_{11}}+0.27 \frac{\bar{v}_{12} \sigma_{12}}{\bar{v}_{22} \sigma_{22}}=1.37 \text {. }
$$

This constitutes a relation between the effective cross section for ammonia-ammonia, ammonia-chloroform, and chloroform-chloroform collisions. We will be satisfied with this result. Our aim was to indicate a new method for the determination of quantities which are usually obtained by measurements at low pressures (see also [Boudouris and Ilias, 1958a and 1958b]) rather than to present new numerical results. Indeed, to obtain precise numerical values, supplementary measurements of a high precision would be needed. This is difficult in the case of chloroform.

Mixtures, ammonia+argon, have also been studied. In order to interpret the behavior of the relative absorption $\alpha_{\text {mix }} / \alpha_{\mathrm{NH}_{3}}$ (curve A of fig. 37) it is only necessary to make $K=0$ in (3.51) and (3.52), the second gas (argon) being not an absorbant. One obtains a relation identical to the one proposed in reference [Battaglia et al., 1958]:

$$
y=\alpha_{\mathrm{mix}} / \alpha_{\mathrm{N} \cdot 3}=\left(1-a_{1 A}\right) c^{2}+a_{1 A} c,
$$

where

$$
a_{1 A}=\bar{v}_{1 A} \sigma_{1 A} / \bar{v}_{11} \sigma_{11},
$$

$\sigma_{1 A}$ is the effective cross section for ammonia-argon collisions. Experiment suggests the value:

$$
a_{1 A}=0.09,
$$

and the parabola becomes:

$$
y=0.91 c^{2}+0.09 c
$$

this curve does go through the measured points (fig. 37). The behavior of the relative absorption curve is very different from the case where the mixture is made up of two constituents, both of them absorbing.

\subsection{Conclusions}

The research done for this dissertation was concerned with the index of refraction, the absorption and the dispersion of energy in the hyperfrequency range by gases and in particular by atmospheric gases. Measurements were made in the following intervals: 7 to $12 \mathrm{Gc} / \mathrm{s}$ for the frequency, 0 to $1 \mathrm{~atm}$ for the gas pressure (except when limitations were imposed by vapors), and 0 to $50{ }^{\circ} \mathrm{C}$ for the temperature.

Part (sec. 3.1) of this work is devoted to a study of the index of refraction of atmospheric gases, dry air and wet air. Our results interpreted with the help of theories resumed in chapter 2 (ClausiusMosotti and Debye-Langevin relations), and some of these are original measurements (krypton, neon, xenon), are compared with results of other authors (tables 1 and 2). A survey of the question is thus realized. The molecular polarization and the dipole moment of water vapor are determined and the results discussed. A series of measurements on dry air and water vapor where the relevant parameters vary between wide limits, contribute to clarify the confusion concerning the coefficients to be used in

Permeability for vacuum
Permittivity for vacuum
Speed of light in vacuum
Boltzmann's constant
Avogardo's constant
Gas constant
Number of molecules per $\mathrm{m}^{3}$
Planck's constant
Pressure
1 Debye unit

Permeability for vacuum Speed of light in vacuum Boltzmann's constant Gas constant $\mu_{0}=4 \pi \times 10^{-7}=1.2566 \times 10^{-6}$ henry $/$ meter. $\epsilon_{0}=1 / \mu_{0} c^{2}=8.8545 \times 10^{-12} \mathrm{farad} /$ meter. $c=2.9979 \times 10^{8} \mu$ sec. $k=1.3804 \times 10^{-23}$ joule $/{ }^{\circ} \mathrm{K}$.

$N_{A}=6.023 \times 10^{26}$ molecules per $M \mathrm{~kg}$. $R=k \times N_{A}=8.314 \times 10^{3}$ joule $^{\circ} \mathrm{K} M_{\mathrm{kg}}$. $N=0.96525 \times 10^{25} \times \frac{p_{i}}{T}\left(p_{i}\right.$ in $\mathrm{mm} \mathrm{Hg}, T$ in $\left.{ }^{\circ} \mathrm{K}\right)$. $H=6.6252 \times 10^{-34}$ joule $\cdot$ second. $760 \mathrm{~mm} \mathrm{Hg} \rightarrow 101.324$ newton/meter. (dipole moment) $\rightarrow 3.3356 \times 10^{-30}$ coulomb - meter. 
the formula which expresses the index of refraction of wet air (3.12).

Another part (sec. 3.2) concerns the absorption and the dispersion. Since we do not have, for the time being, the necessary frequency bands required for a direct study of atmospheric gases, we have used other gases, ammonia and chloroform. These results, however, are of interest for the propagation of microwave energy in the atmosphere. This point has been made in section $3.2 \mathrm{~A}$ and following. The interpretation of the results has been made with the help of the Van Vleck-Weisskopf theory which has been presented and discussed in chapter 2 . For the case of ammonia we have studied the representation of the absorption by a unified line ((3.17) and fig. 28), the dependence of the absorption on the temperature $\left(T^{-2.5}\right.$ law), the variation of the slope of the index of refraction as the function of pressure, and we have indicated how those results can be compared to the case of oxygen. The absorption by chloroform, studied here for the first time at average pressure and with more details than usual, was the object of a discussion concerning the theory. The influence of a large number of rotation lines is noted and discussed. A $T^{-4}$ law for the variation of the absorption as a function of temperature is established. A few qualitative conclusions are presented concerning dispersion for the complicated case of chloroform. Generally, although certain reservations have to be made, the formulas of $\mathrm{VV}-\mathrm{W}$ represent more or less the general behavior of these phenomena. A new method of research by means of gaseous mixtures has been sketched (sec. 3.2F).

The experimental setup which we have used was discussed in chapter 1 . The original method of Gozzini according to which the responses of the cavities are interpreted by pulse techniques, the use of specially-designed cavities, the careful construction of the instrument and the numerous improvements from which it has benefitted, have made this apparatus an excellent research tool particularly welladapted to the study of the index of refraction, the dispersion and the absorption by gases at average pressure in the hyperfrequency range.

Our sincere thanks to Mrs. Anna von Kreisler for her extensive help in the preparation of the translation of this paper.

\section{References}

Anderson, P. W. (1949a), The theory of pressure broadening of spectral lines in the microwave and infra-red region, Thesis (Harvard).

Anderson, P. W. (May 1949b), On the anomalous line-shapes in the ammonia inversion spectrum at high pressures, Phys. Rev. 75, p. 1450.

Anderson, P. W. (Sept. 1949c), Pressure broadening in the microwave and infra-red regions, Phys. Rev. $76,647-661$.

Anderson, P. W. (Nov. 1950), Pressure broadening of the ammonia inversion line by foreign gases: quadrupoleinduced dipole interactions, Phys. Rev. 80, 511-513.

Anderson, P. W. (June 1952), A method of synthesis of the statistical and impact theories of pressure broadening, Phys. Rev. 86, p. 809.
Arnot, F. L. (1950), Collision process in gases (Methuen and Co. Ltd., London).

Artman, J. O. (June 1, 1953), Absorption of microwaves by oxygen in the millimeter wavelength region, Report $\mathrm{CC}_{0}-$ lumbia Rad. Lab., Columbia Univ. Div. of Govern. Aided Res., New York 27, New York).

Artman, J. O., and J. P. Gordon (Dec. 1954), Absorption of microwaves by oxygen in the millimeter wavelength region, Phys. Rev. 96, 1237-1245.

Barrell, H. (May 1951), The dispersion of air between 2500A and 6500A, J. Opt. Soc. Am. 41, p. 295.

Barrell, H., and J. E. Sears (1939), On the refraction and dispersion of air for the visible spectrum, Phil. Trans. Roy. Soc. A238, 2-64.

Battaglia, A., G. Boudouris, F. Bruin, A. Gozzini, and D. Ilias (Aug. 1958), Microwave spectrometer based on cavity and pulse technique, Commun. Congres Intern., Hyperfr., held in Paris (Oct. 1957), Onde El., Suppl. Spec. 2, 430-440.

Battaglia, A., G. Boudouris, and A. Gozzini (May 1957), Sur l'indice de rèfraction de l'air humide en microondes, Ann. Tèlec. 12, 47-50.

Battaglia, A. F., F. Bruin, and A. Gozzini (Jan. 1958), Mierowave apparatus for the measurement of the refraction, dispersion and absorption of gases at relatively low pressure, Nuovo Cimento $\boldsymbol{\gamma}$, Sèrie X, 1-9.

Battaglia, A., and A. Gozzini (May 1955), Un appareil pour

l'ètude de la dispersion des gaz et des vapeurs, dans la bande des hyperfrèquences, Onde El. 35, 500-502.

Becker, G. E., and S. H. Autler (Sept. 1946), Water vapor absorption of electromagnetic radiation in the centimeter wave-length range, Phys. Rev. Y0, 300-307.

Beringer, R. (Jan. 26, 1945), The absorption of one-half centimeter waves in oxygen, R. L. Report 684 M.I.T.

Beringer, R. (July 1946), The absorption of one-half centimeter electromagnetic waves in oxygen, Phys. Rev. $\boldsymbol{y}_{0}$ $53-57$.

Beringer, R., and J. G. Castle (Jan. 1951), Microwave mag netic resonance spectrum of oxygen, Phys. Rev. 81, 82-88.

Birnbaum, G. (July 1949), Dispersion in $\mathrm{NH}_{3}$ in the microwave region, Phys. Rev. $\boldsymbol{\gamma 6}, 178-179$.

Birnbaum, G. (Jan. 1950), Dispersion in $\mathrm{NH}_{3}$ in the microwave region, Phys. Rev. $\mathbf{9 y , ~ 1 4 4 - 1 4 5 . ~}$

Birnbaum, G. (Jan. 1953), Millimeter wavelength dispersion of water vapor, J. Chem. Phys. 21, 57-61.

Birnbaum, G., and S. K. Chatterjee (Feb. 1952), The dielectric constant of water vapor in the microwave region, J. Appl. Phys. 23, 220-223.

Birnbaum, G., S. J. Kryder, and S. H. Lyons (Jan. 1951), Microwave measurements of the dielectric properties of gases, J. Appl. Phys. 22, 95-102.

Birnbaum, G., and A. A. Maryott (Oct. 1953a), Absorption in the low-frequency wing of the $\mathrm{NH}_{3}$ inversion spectrum, J. Chem. Phys. 21, 1774-1777.

Birnbaum, G., and A. A. Maryott (Oct. 1953b), Change in the inversion spectrum of $\mathrm{ND}_{3}$ from resonant to nonresonant absorption, Phys. Rev. 92, 270-273.

Birnbaum, G., and A. A. Maryott (Aug. 1954), Temperature dependence of the microwave absorption of $\mathrm{NH}_{3} \mathrm{~J}$. Chem. Phys. 22, p. 1457.

Bleaney, B., and J. H. N. Loubser (April 1948), Collision broadening of the ammonia inversion spectrum at high pressures, Nature 161, 522-523.

Bleaney, B., and J. H. N. Loubser (May 1950), The inversion spectra of $\mathrm{NH}_{3}, \mathrm{CH}_{3} \mathrm{Cl}$ and $\mathrm{CH}_{3} \mathrm{Br}$ at high pressures, Proc. Phys. Soc. A63, 483-493.

Bleaney, B., J. H. N. Loubser, and R. P. Penrose (Mar. 1947), Cavity resonators for measurements with centimetre electromagnetic waves, Proc. Phys. Soc. 59, 185-199.

Bleaney, B., and R. P. Penrose (May 1947a), The inversion spectrum of ammonia at centimetre wave-lenths, Proc. Roy. Soc. 189, 358-371.

Bleaney, B., and R. P. Penrose (May 1947b), Collision broadening of the inversion spectrum of ammonia at centimetre wave-lengths, I. Self-broadening at high pressure, Proc. Phys. Soc. (London) 59, 418-428.

Bleaney, B., and R. P. Penrose (Jan. 1948a), Pressure broadening of the inversion spectrum of ammonia, part II. Disturbance of thermal equilibrium at low pressures, Proc. Phys. Sos. (London) 60, 83-98. 
Bleaney, B., and R. P. Penrose (June 1948b), Collision broadening of the inversion spectrum of ammonia, III. The collision cross-sections for self-broadening and for mixtures with non-polar gases, Proc. Phys. Soc. (London) 60, 540549 .

Boudouris, G. (1957), Propagation troposphérique, ed. C.D.U. (Paris).

Boudouris, G., and D. Ilias (Feb. 1958a), Sur l'absorption en microondes de quelques mélanges gazeux, Compt. rend. 246, 730-733.

Boudouris, G., and D. Ilias (Mar. 1958b), Mesures d'absorption des microondes par des mèlanges gazeux binaires, Compt. rend 246, $1407-1410$.

Bruhat, G. (1954), Optique, ed. revue et complétée par A. Kastler, ed. (Masson, Paris).

Bruin, F. (July 1956), The line shape of collision broadened spectral absorption lines, Thesis (Univ. d'Amsterdam).

Burton, W. (May 1908), The refractive index and dispersion of light in argon and helium, Proc. Roy. Soc. A80, 390-405.

Carrara, N. (1956), Spettroscopia molecolare dell'ossigeno per mezzo di microonde, Forma delle righe di assorbimento, Nuovo Cimento. Suppl. 4, Série X, 1593-1608. In Italian.

Clay, J., and F. Van der Maesen (July 1949), The absolute dielectric constant of gases at pressures of $0-80 \mathrm{~atm}$. at $25^{\circ} \mathrm{C}$, Physica 15, $467-483$.

Cleeton, C. E., and N. H. Williams (Feb. 1934), Electromagnetic waves of $1.1 \mathrm{~cm}$ wavelength and the absorption spectrum of ammonia, Phys. Rev. 45, p. 234

Crain, C. M. (Sept. 1948), The dielectric constant of several gases at a wave-length of 3.2 centimeters, Phys. Rev. $\mathbf{y}$, 691-693.

Crain, C. M. (Feb. 1952), Discussion on the permittivity of air at a wavelength of 10 centimeters by W. E. Phillips, Proc. IRE 40, p. 164

Cuthbertson, C., and M. Cuthbertson (Feb. 1932), The refraction and dispersion of neon and helium, Proc. Roy. Soc. A135, $40-47$.

Debye, P. (Feb. 1912), Results of kinetic theory of dielectrics, Physik Z. 13, 97-100.

Debye, P. (1925), Handbuch der radiologie (Marx) (Akad. Verlag. M.B.H., Leipzig).

Debye, P. (1929), Polar Molecules (Chem. Catal. Col., New York).

Debye, P. (1945), Polor Molecules (Dover Publ., New York).

Debye, P. (1954), The Collected Papers of P. Debye (Interscience Publ., New York).

Essen, L. (Jan. 1953a), A highly stable microwave oscillator and its application to the measurement of the spatial variations of refractive index in the atmosphere, PIEE, III 100, $19-24$.

Essen, L. (Mar. 1953b), The refractive indices of water vapour, air, oxygen, nitrogen, hydrogen, deuterium, and helium, Proc. Phys. Soc. (London) B 66, 189-193.

Essen, L., and K. D. Froome (Oct. 1951), The refractive indices and dielectric constants of air and its principal constituent at $24,000 \mathrm{Mc} / \mathrm{s}$, Proc. Phys. Soc. (London) 64, 862-875.

Essen, L., and K. D. Froome (Mar. 1952), The measurement of the velocity of electromagnetic waves and the refractive index of air at microwave frequenices, Nuovo Cimemto 9 Suppl., 277-282.

Foley, H. M. (1942), Thesis (Univ. of Michigan, Ann Arbor, Mich.)

Foley, H. M. (June 1946), The pressure broadening of spectral lines, Phys. Rev. 69, 616-623.

Froome, K. D. (Nov. 1955), The refractive indices of water vapour, air, oxygen, nitrogen, and argon at $72 \mathrm{k} \mathrm{Mc} / \mathrm{s}$ Proc. Phys. Soc. (London) 68, 833-835.

Froome, K. D. (Sept. 1956), Microwave determinations of the velocity of "light," J. Brit. Inst. Radio Eng. 16, 497513.

Frevmann, R. (1936), Ondes hertziennes-structure moléculaire, 2 vol. (Hermann, Paris).

Fröhlich, H. (April 1946), Shape of collision, broadened spectral lines, Nature 15\%, p. 478.

Fröhlich, H. (April 1948), General theory of the static dielectric constant, Trans. Faraday Soc. 44, 238-243.

Fröhlich, H. (1949), Theory of Dielectrics (Clarendon Press, Oxford, England).

Fröhlich, H. (Oct. 1956), Remark on the calculation of the static dielectric constant, Physica 22, 898-904.
Gennaoui, A. (May 1951), Measurement of the dielectric constants and the absorption coefficients of gases and vapours at hyperfrequencies (microwave frequencies), Helv. Phys. Acta 24, 401-418. In French.

Gennaoui, A., R. Lacroix, and C. Ryter (1949), Dielectric constant of gases, Arch. Sci. 2, 371-374 (in French).

Ghosh, S. N., R. Trambarulo, and W. Gordy (Apr. 1952), Microwave spectra and molecular structures of fluoroform, chloroform, and methyl chloroform, J. Chem. Phys. 20, $605-607$.

Gordy, W., W. V. Smith, and R. Trambarulo (1953), Microwave Spectroscopy (John Wiley, New York; Chapman, London).

Goudet, G., and P. Chavance (1955), Ondes centimètriques (Chiron, Paris).

Gozzini, A. (June 1951), The dielectric constants of gases in the microwaveregion, Nuovo Cimento 8,361-368 (in Italian).

Gozzini, A., and E. Polaceo (1953a), Mèthodè pour la mesure de la constante dièlectrique des gaz dans la bande des hyperfrequences; Compt. rend $\mathbf{2 3}$, 1497-1499.

Gozzini, A., and E. Polacco (1953b), Complèment a la mèthode de mesure de la constante, Compt. rend. 237, 1652-1654.

Groves, I. G., and S. Sugden (1935), Dipole moments of vapors, Part 2, J. Chem. Soc. (London), 971-974.

Guèrin, H. (1952), Traitè de manipulation et d'analyse des gaz (ed. Masson, Paris).

Hector, L. G., and D. L. Woernley (Feb. 1946), The dielectric constants of ejght gases, Phys. Rev. 69, 101-105.

Heineken, F. W., and F. Bruin (June 1954), Some measurements on refractive indices of gases in the microwave region, Physica 20, 350-360.

Heineken, F. W., and H. W. Wijn (July 1958), Communication au Colloque Intern. A.M.P.E.R.E. (Paris).

Heitler, W. (1954), The Quantum Theory of Radiation, 3d ed. (Oxford Univ. Press, London).

Hershberger, W. D. (June 1946), The absorption of microwaves by gases, J. Appl. Phys. 17, 495-500.

Herzberg, G. (1951), Molecular Spectra and Molecular structure (H. Van Nostrand, New York)

Hill, R. M., and W. V. Smith (May 1950), Microwave collision diameters and associated quadrupole moments, Phys. Rev. 82, p. 451

Hill, T. L. (Jan. 1958), Theory of the dielectric constant of imperfect gases and dilute solutions, J. Chem. Phys. 28, 61-66.

Holstein, T. (Aug. 1950), Pressure broadening of spectral lines, Phys. Rev. 79, p. 744.

Howard, R. R., and W. V. Smith (Mar. 1950a), Temperature dependence of microwave line widths, Phys. Rev. $\boldsymbol{y}, 840$ 841.

Howard, R. R., and W. V. Smith (July 1950b), Microwave collision diameters, I. Expeıimental, Phys. Rev. 99, 128131.

Hughes, J. V., and H. L. Armstrong (May 1952), The dielectric constant of dry air, J. Appl. Phys. 23, 501-504.

Hurdis, E. C., and C. P. Smyth (1942), Dipole moment induction and resonance in nitroethane and some chloronitroparaffins, J. Am. Chem. Soc. 64, 2829-2834.

Ingram, D. J. E. (1955), Spectroscopy at radio and microwave frequencies (Butterworths Sci. Public., London).

Ishiguro, E., T. Arai, M. Mizushima, and M. Kotani (March 1952), On the polarizability of the hydrogen molecule, Proc. Phys. Soc. (London) A65, 178-187.

Jablonski, A. (June 1940), Pressure broadening of spectra I lines, Physica \%, 541-551.

Jablonski, A. (Aug. 1945), General theory of pressure broadening of spectral lines, Phys. Rev. 68, 78-93.

Jablonski, A. (Jan. 1946), Errata: General theory of pressure broadening of spectral lines, Phys. Rev. 69, p. 31

Jablonski, A. (Feb. 1948), On the phase shift approximation in the theory of pressure broadening of spectral lines, Phys . Rev. $\mathbf{7 3}, 258-259$.

Jansen, L., and P. Mazur (March 1955), On the theory of molecular polarization in gases. I. Effect of molecular interactions on the polarizability of spherical nonpolar molecules, Physica 21, 193-207.

Jansen, L., and A. D. Solem (Dec. 1956), Molecular theory of the dielectric constant of nonpolar gases, Phys. Rev. 104, 1291-1297. 
Jelatis, J. G. (April 1948), Measurements of dielectric constant and dipole moment of gases by the best-frequency method, J. Appl. Phys. 19, 419-425.

Jen, C. K. (Nov. 1947), Microwave spectra and the Zeeman effect in a resonant cavity absorption cell, Phys. Rev. 72, p. 986.

Jen, C. K. (July 1948), A method for measuring the complex dielectric constant of gases at microwave frequencies by using a resonant cavity, J. Appl. Phys. 19, 649-653.

Karplus, R., and J. Schwinger (May 1948), A note on saturation in microwave spectroscopy, Phys. Rev. 73, 1020-1026.

Kaye, G. W. G., and T. H. Laby (1948), Tables of Physical and Chemical Constants (Longmans-Green Co., London). Kennard, E. H. (1938), Kinetic Theory of Gases (McGrawHill Co., New York).

Kerr, D. E. (1951), Propagation of Short Radio Waves, Rad. Lab. Series No. 13 (McGraw-Hill Co., New York).

Krishnaji, H., and P. Swarup (Jan. 1953a), Collision broadening of the microwave spectrum of ammonia, J. Sci. Ind. Research (India) 12B, 1-3.

Krishnaji, H., and P. Swarup (Aug. 1954), Temperature dependence of microwave absorption coefficient, J. Chem. Phys. 22, 1456-1457.

Krishnaji, H., and P. Swarup (Dec. 1953b), Absorption coefficients at $3.06-3.44 \mathrm{~cm}$ and at pressures of $20-76 \mathrm{~cm}$ $\mathrm{Hg}$ for $\mathrm{NH}_{3}, \mathrm{C}_{2} \mathrm{H}_{5} \mathrm{Cl}, \mathrm{CH}_{3} \mathrm{CHO}, \mathrm{CH}_{3} \mathrm{I}$ and $\left(\mathrm{CH}_{3}\right)_{2} \mathrm{CO}, \mathrm{J}$. Appl. Phys. 24, p. 1525.

Kuhn, H. (Nov. 1934), Pressure shift and broadening of spectral lines, Phil. Mag. (7) 18, 983-987.

Kuhn, H. (1937), Pressure broadening of spectral lines and Van der Waals forces, Proc. Roy. Soc. A158, 212-241.

Kuhn, H., and F. London (Nov. 1934), Limitation of the potential theory of the broadening of spectral lines, Phil Mag. (7) 18,

983-987.

Lacroix, R., and C. Ryter (Mar. 1950), Methodé différentielle pour mesurer la variation de fréquence propre d'une cavité, Arch. Sci. 3, 165-174.

Lenz, W. (1933), Allgemeine Theorie der Verbreiterung von Spektralinien, Z. Physik 80, 423-447.

Leslie, D. C. M. (1951), II. Collision broadening at microwave frequencies, Phil. Mag. (7) 42, 37-55.

Lindholm, E. (1941), Zur Theorie der Verbreiterung von Spektralinien, Ark. Mat. Astron. och. Fysik 28B, No. 3, $1-11$.

Lindholm, E. (1942), Thesis (Uppsala College, Uppsala, New Jersey).

Lindholm, E. (1945), Pressure broadening of spectral lines, Ark, Mat. Astron. och Fysik 32A, No. 17, 1-18.

Lorentz, H. A. (Jan. 1906), Absorption and emission lines of gases, Kon. Akad. Wetensch. Amsterdam, Proc. 8, 591-611.

Lorentz, H. A. (1909), Theory of electrons (Teubner Verlagsgesellschaft, Leipzig; Stechert and Co., New York, 1916).

Loubser, J. H. N., J. Klein, and G. H. Townes (Sept. 30, 1949), Measurements of the absorption of millimeter waves in $\mathrm{ND}_{3}$, Columbia Rad. Phys. Dept., Columbia Univ. Quart. Progr. Rept.

Lyons, H., G. Birnbaum, and S. J. Kryder (Nov. 1948), Measurements of the complex dielectric constant of gases

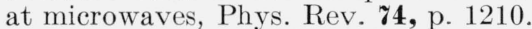

Mandel, M. (July 1958), Commun. au Colloque Intern., A.M.P.E.R.E. (Paris).

Mandel, M., and P. Mazur (1958), On the molecular theory of dielectric polarization, Physica $\mathbf{2 4}, 116-128$

Margenau, H. (Nov. 1935), Theory of pressure effects of foreign gases on spectral lines, Phys. Rev. 48, 755-765.

Margenau, H. (July 1949a), Pressure dependence of the inversion frequency of $\mathrm{NH}_{3}$, Phys. Rev. $\mathbf{7 6}, 585-586$.

Margenau, H. (Nov. 1949b), Inversion frequency of ammonia and molecular interaction, Phys. Rev. 76, 1423-1429.

Margenau, H. (April 3, 1956), The validity of the statistical theory of pressure broadening, RM-1670-AEC (Rand Corp., Santa Monica, Calif.).

Margenau, H., and S. Bloom (July 1950), Pressure broadening of spectral lines and frequency modulation, Phys. Rev. 79, p. 213.

Margenau, H., and W. W. Watson (Jan. 1936), Pressure effects on spectral lines, Rev. Mod. Phys. 8, 22-53.

Maryott, A. A.., and G. Birnbaum (Sept. 1955), Microwave absorption in compressed oxygen, Phys. Rev. 99, p. 1886.
Mascart, C. (1874), Réfraction de l'eau comprimé, Compt. rend. 78, 617-619.

Mascart, C. (1878), Réfraction et dispersion de vapeurs, Compt. rend. 86, $1182-1186$.

Michels, A., and C. Michels (June 1932), The dielectric constant of nitrogen up to 150 atmospheres at $25^{\circ}, 75^{\circ}$, and $125^{\circ} \mathrm{C}$, Phil. Mag. 13, 1192-1196.

Michels, A., C. A. Ten Seldam, and S. D. J. Overdijk (Aug. 1951), The dielectric constant of argon at $25^{\circ} \mathrm{C}$ and $125^{\circ} \mathrm{C}$ for pressures up to 2700 atmospheres, Physica 1\%, 781-787.

Mazur, P., and M. Mandel (April 1956a), On the theory of the refractive index of non-polar gases, Part 1. Quantum mechanical part, Physica 22, 289-298.

Mazur, P., and M. Mandel (April 1956b), On the theory of the refractive index of non-polar gases, Part 2. Statistical part, Physica 22, 299-310.

Millman, G. H., and R. C. Raymond (April 1949), The absorption of microwaves in gases, J. Appl. Phys. 20, p. 413.

Mizushima, M. (July 1951a), The theory of pressure broadening and its application to microwave spectra, Phys. Rev. 83, 94-103.

Mizushima, M. (Oct. 1951b), Erratum: The theory of pressure broadening and its application to microwave spectra, Phys. Rev. 84, p. 363.

Montgomery, C. G. (1947), Technique of microwave measurements, MIT Rad. Lab. Series No. 11 (McGraw-Hill Co., New York).

Montgomery, C. G., R. H. Dijke, and E. M. Purcell (1948), Principles of microwave circuits, MIT Rad. Lab. Series No. 8 (McGraw-Hill Co., New York).

Murphy, J. A., and R. C. Ravmond (Oct. 1950), Dielectric constants of five gases at $9400 \mathrm{Mc} / \mathrm{s}$, J. Appl. Phys. 21, 1064-1065.

Pauling, L., and E. B. Wilson, Jr., (1935), Introduction to Quantum Mechanies (McGraw-Hill Co., New York).

Philips, W. E. (July 1950), The permittivity of air at a wavelength of ten centimeters, Proc. IRE 38, 786-790.

Rentschler, H. C. (1908), New method for measuring the index of refraction of a gas for different light-waves. Astrophys. J. 28, 345-359

Rogers, T. F. (Oct. 1951), Factors affecting the width and shape of atmospherique microwave absorption lines, Rept. No. E 5078 (A. F. Cambridge Res. Cen., Mass.)

Ruark, A. E., and H. C. Urey (1930), Atoms, Molecules, Quanta (MeGraw-Hill Co., New York).

Saito, S. (Aug. 1955), Measurement at 9,000 Me of the dielectric constant of air containing various quantities of water vapor, Proc. IRE 43, p. 1009.

Sanger, R. (1930), Bestimmung des elektrischen Momentes eines Molekuls aus dem Temperaturverhalten der Dielektrizitätskonstanten, Physik. Z. 31, 306-315.

Sanger, R. (1932), Temperatureffekt der Molekularpolarisation einiger Gase und Dämpfe, Helv. Phys. Acta 5, 200-210.

Saxton, J. A. (1943), The dielectric constant and absorption coefficient of water vapour for wavelengths of $9 \mathrm{~cm}$ and $3.2 \mathrm{~cm}$, Nat. Phys. Lab. (Teddington, England) Paper No. RRB/S 11.

Saxton, J. A. (1944), The dielectric constant and absorption coefficient of water vapour for the wavelength of $1.6 \mathrm{~cm}$, Nat. Phys. Lab. (Teddington, England) Paper No. RRB/S 17

Saxton, J. A. (1947), The dielectric properties of water vapor at very high radio frequencies. Meteorological factors in radio-wave propagation (The Phys. Soc., London).

Schiff, L. I. (1949), Quantum Mechanies (McGraw-Hill, New York).

Smith, E. K., and S. Weintraub (Jan. 1953a), The constants in the equation for atmospheric refractive index at radio frequencies, J. Res. NBS 50, 39-41 RP2385.

Smith, E. K., and S. Weintraub (Aug. 1953b), The constants in the equation for atmospheric refractive index at radio frequencies, Proc. IRE 41, 1035-1037.

Smith, D. F. (Aug. 1948), Pressure broadening in ammonia at centimeter wave-lengths, Phys. Rev, 74, 506-507.

Smith, W. V., (Sept. 1956), Evaluation of molecular quadrupole moments from microwave spectral line breadths, I. Theoretical, J. Chem. Phys. 25, 510-515.

Smith, W. V., and R. Howard (July 1950), Microwave collision diameters, II. Theory and correlation with molecular quadrupole moments, Phys. Rev. 89, 132-136. 
Smith, W. V., and R. Unterberger (Dec. 1949), Microwave investigations of chlorofrom, J. Chem. Phys. 17, p. 1348.

Smyth, C. P. (1955), Dielectric Behavior and Structure (dielectric constant and loss, dipole moment and molecular structure) (McGraw-Hill Co., New York).

Stickland, A. C. (May 11, 1942), The dielectric constant of water vapour and its effect upon the propagation of very short waves, RRB 1594, Radio Dept. (Nat. Phys. Lab., Dept. Sc. Ind. Res., Teddington, England).

Stranathan, J. D. (Sept. 1935), Dielectric constant of water vapor, Phys. Rev. 48, 538-544.

Strandberg, M. P. W., C. Y. Meng and J. G. Ingersoll (May 1949), Phys. Rev. 75, 1524.

Stratton, J. A. (1941), Electromagnetic Theory (McGrawHill Co., New York).

Swarup, P. (June 1954), Temperature dependence of microwave absorption coefficient, J. Sci. Indust. Res. (India) 13B, 389-392.

Tausz, J., and G. Hornung (1927), Refraction of light in gases, Z. tech. Physik. 8, 338-355.

Tinkham, M., and M. W. P. Strandberg (July 1955), Line breadths in the microwave magentic resonance spectrum, Phys. Rev. 99, 537-539.

Townes, C. H. (Nov. 1946), The ammonia spectrum and line shapes near $1.25 \mathrm{~cm}$ wave-length, Phys. Rev. 90, 665-671.

Townes, C. H., and A. L. Schalow (1955), Microwave Spectroscopy (McGraw-Hill Co., New York).

Unterberger, R. R., R. Trambarulo, and W. V. Smith (April 1950), Microwave determination of the structure of choloroform, J. Chem. Phys. 18, 565-566.

Van Itterbeek, A., and K. de Clippeleier (June 1946), Mesures sur la constante diélectrique de l'anhydride carbonique de l'ammoniaque ainsi que de mélanges, Physica 12, 97-104.

Van Vleck, J. H. (1932), The Theory of Electric and Magnetic Susceptibilities (Clarendon Press, Oxford).

Van Vleck, J. H. (April 1947), The absorption of microwaves by oxygen, Phys. Rev. $\boldsymbol{1 1}, 413-424$.

Van Vleck, J. H., and H. Margenau (Aug. 1949a), Collision theories of pressure broadening of spectral lines, Phys. Rev. $\mathbf{7 6 ,}$ p. 585.
Van Vleck, J. H., and H. Margenau (Oct. 1949b), Collision theories of pressure broadening of spectral lines, Phys. Rev ;6, $1211-1214$

Van Vleck, J. H., and V. F. Weisskopf (April-July 1945), On the shape of collision-broadened lines, Rev. Mod. Phys. 17, $227-236$.

Vassy, E. (1956), Physique de l'Atmosphère, I. (GauthierVillars, Paris).

Walter, J. E., and W. D. Hershberger (Oct. 1946), Absorption of microwaves by gases, II., J. Appl. Phys. 17, 814-822.

Watson, H. E., G. G. Rao, and K. L. Ramaswamy (Aug. 1931), The dielectric coefficients of gases, Part I. The rare gases and hydrogen, Proc. Roy. Soc. A132, 569-585.

Watson, H. E., G. G. Rao, and K. L. Ramaswamy (Feb. 1934), The dielectric coefficients of gases, Part II. The lower hydrides of carbon and silicon, oxygen, nitrogen, oxides of nitrogen and carbon, and fluorides of silicon and sulphur, Proc. Roy. Soc. A143, 558-588.

Weidner, R. T. (Dec. 1947), The microwave spectrum of iodine monochloride at $4 \frac{1}{2}$ centimeters wavelength, Phys. Rev. 7\%, 1268-1269.

Weidner, R. T. (Feb. 1948), Nuclear quadrupole interaction with IC1 rotational spectrum, Phys. Rev. 73, p. 254.

Weingarten, I. R. (May 1, 1948), The absorption of microwaves by gases at high pressure, Thesis, Columbia Rad. Lab. Rept. (Columbia Univ., New York).

Weiss, M. T., and M. W. P. Strandberg (Aug. 1951), The microwave spectra of the deutero-ammonias, Phys. Rev. 83, $567-575$.

Weisskopf, V. (1932), Zur. Theorie der Kopplungsbrette und der StoBdāmpfung, Z. Physik 75, 287-301.

Zieman, C. M. (Jan. 1952), Dielectric constants of various gases at $9470 \mathrm{Mc}$, J. Appl. Phys. 23, p. 154.

(Paper 67D6-297) 